\title{
Base-Promoted Stereoselective Hydrogenation of Ynamides with Sulfonyl Hydrazide to Give $Z$-Enamides
}

Zemin Zhao, Qingyu Tian, Yanhui Chen, Si Wen, Yuqing Zhang, and Guolin Cheng*

Xiamen Key Laboratory of Optoelectronic Materials and Advanced Manufacturing, College of Materials Science and Engineering, the Instrumental Analysis Center, Huaqiao University, Xiamen, Fujian 361021, China

glcheng@hqu.edu.cn

Table of Contents

1. Heating plate

2. Optimization of Reaction Conditions S3

3. ${ }^{1} \mathrm{H}$ and ${ }^{13} \mathrm{C}$ NMR Spectra S5

4. Crystallogiphic Date S46 


\section{Heating plate}
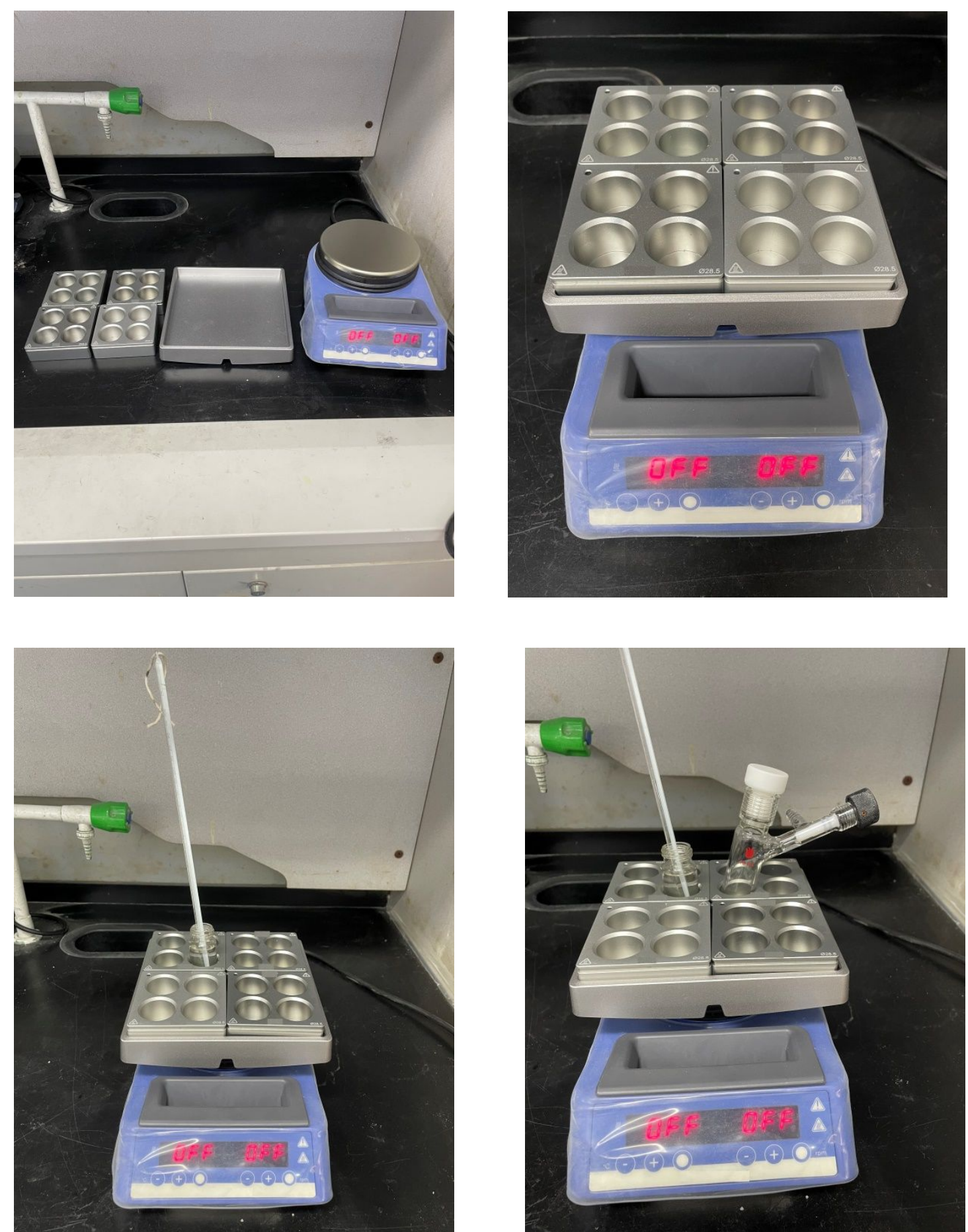

\section{Optimization of Reaction Conditions}

2.1 Table S1 Optimization of the Reaction Conditions ${ }^{\mathrm{a}}$ 


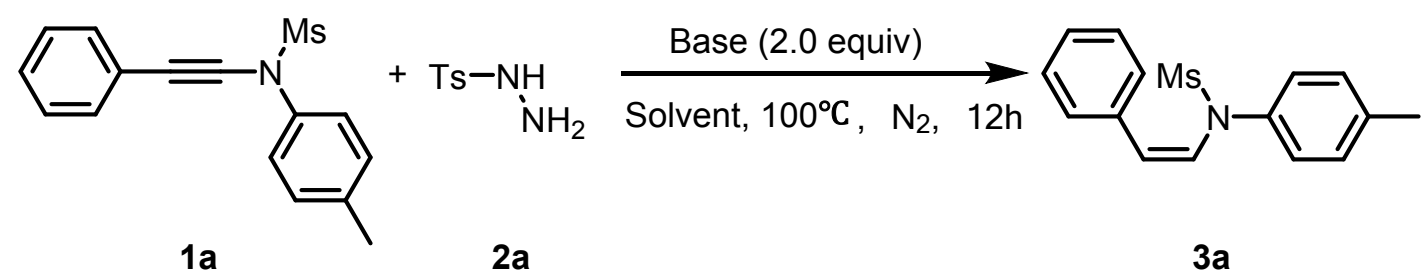

\begin{tabular}{|c|c|c|c|}
\hline Entry & Solvent & Base & Yield $[\%]^{\mathrm{b}}$ \\
\hline 1 & $\mathrm{DMF}$ & $\mathrm{K}_{2} \mathrm{CO}_{3}$ & 50 \\
\hline 2 & Acetonitrile & $\mathrm{K}_{2} \mathrm{CO}_{3}$ & 44 \\
\hline 3 & toluene & $\mathrm{K}_{2} \mathrm{CO}_{3}$ & 36 \\
\hline 4 & DMSO & $\mathrm{K}_{2} \mathrm{CO}_{3}$ & 68 \\
\hline 5 & acetone & $\mathrm{K}_{2} \mathrm{CO}_{3}$ & trace \\
\hline 6 & DCE & $\mathrm{K}_{2} \mathrm{CO}_{3}$ & trace \\
\hline 7 & THF & $\mathrm{K}_{2} \mathrm{CO}_{3}$ & 41 \\
\hline 8 & DMA & $\mathrm{K}_{2} \mathrm{CO}_{3}$ & 51 \\
\hline 9 & NMP & $\mathrm{K}_{2} \mathrm{CO}_{3}$ & 35 \\
\hline 10 & DMPU & $\mathrm{K}_{2} \mathrm{CO}_{3}$ & trace \\
\hline 11 & Dioxane & $\mathrm{K}_{2} \mathrm{CO}_{3}$ & 30 \\
\hline 12 & MTBE & $\mathrm{K}_{2} \mathrm{CO}_{3}$ & 33 \\
\hline 13 & HFIP & $\mathrm{K}_{2} \mathrm{CO}_{3}$ & trace \\
\hline 14 & tert-butanol & $\mathrm{K}_{2} \mathrm{CO}_{3}$ & 68 \\
\hline 15 & tert-butanol & ${ }^{t} \mathrm{BuONa}$ & 56 \\
\hline 16 & tert-butanol & ${ }^{t} \mathrm{BuOK}$ & 48 \\
\hline 17 & tert-butanol & DBU & 66 \\
\hline 18 & tert-butanol & Pytidine & 51 \\
\hline 19 & tert-butanol & Triethylamine & 45 \\
\hline 20 & tert-butanol & $\mathrm{NaOH}$ & 54 \\
\hline 21 & tert-butanol & $\mathrm{KOH}$ & 53 \\
\hline 22 & tert-butanol & $\mathrm{K}_{3} \mathrm{PO}_{4}$ & 45 \\
\hline 23 & tert-butanol & $\mathrm{NaH}_{2} \mathrm{PO}_{4}$ & 48 \\
\hline 24 & tert-butanol & KOAc & 40 \\
\hline 25 & tert-butanol & $\mathrm{NaOAc}$ & 70 \\
\hline 26 & tert-butanol & LiOAc & 68 \\
\hline
\end{tabular}




\begin{tabular}{|c|c|c|c|}
\hline 27 & tert-butanol & $\mathrm{Na}_{2} \mathrm{CO}_{3}$ & 74 \\
\hline 28 & tert-butanol & $\mathrm{Li}_{2} \mathrm{CO}_{3}$ & trace \\
\hline 29 & tert-butanol & $\mathrm{Cs}_{2} \mathrm{CO}_{3}$ & trace \\
\hline 30 & tert-butanol & $\mathrm{NaHCO}_{3}$ & 41 \\
\hline 31 & tert-butanol & $\mathrm{KHCO}_{3}$ & 66 \\
\hline 32 & tert-butanol & - & 12 \\
\hline $33^{c}$ & tert-butanol & $\mathrm{Na}_{2} \mathrm{CO}_{3}$ & 78 \\
\hline $34^{\mathrm{d}}$ & tert-butanol & $\mathrm{Na}_{2} \mathrm{CO}_{3}$ & 74 \\
\hline $35^{\mathrm{e}}$ & tert-butanol & $\mathrm{Na}_{2} \mathrm{CO}_{3}$ & 40 \\
\hline $36^{\mathrm{f}}$ & tert-butanol & $\mathrm{Na}_{2} \mathrm{CO}_{3}$ & trace \\
\hline
\end{tabular}

\subsection{Table S2 Optimization of the Reaction Conditions ${ }^{\mathrm{a}}$}

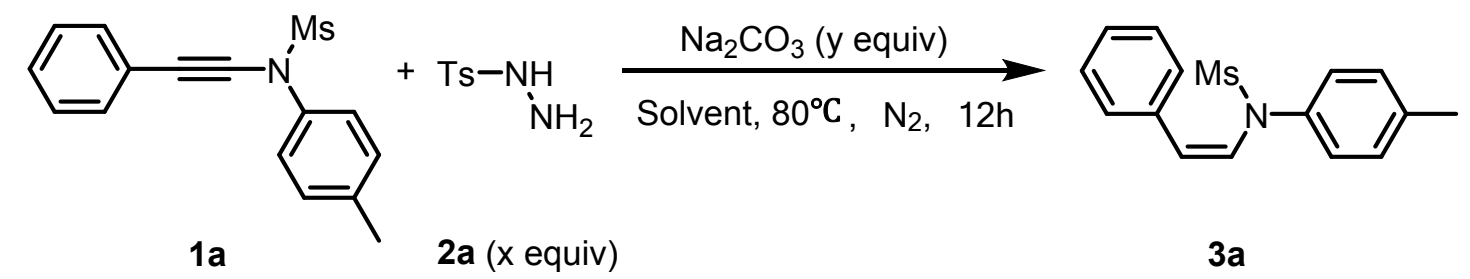

\begin{tabular}{lllll}
\hline Entry & Solvent & $\mathbf{2}$ (x equiv) & $\mathrm{Na}_{2} \mathrm{CO}_{3}$ (y equiv) & Yield [\% $]^{\mathrm{b}}$ \\
\hline 1 & tert-butanol & $(1.0$ equiv) & $(2.0$ equiv) & 56 \\
2 & tert-butanol & $(1.5$ equiv) & $(2.0$ equiv) & 66 \\
3 & tert-butanol & $(2.5$ equiv) & $(2.0$ equiv) & 84 \\
4 & tert-butanol & $(2.0$ equiv) & $(1.0$ equiv) & 76 \\
5 & tert-butanol & $(2.0$ equiv) & $(1.5$ equiv) & 85 \\
6 & tert-butanol & $(2.0$ equiv) & $(2.5$ equiv) & 72 \\
7 & tert-butanol & $(2.5$ equiv) & $(1.5$ equiv $)$ & 74 \\
$8^{c}$ & tert-butanol & $(2.0$ equiv) & $(1.5$ equiv $)$ & 40 \\
\hline
\end{tabular}

a $1 \mathbf{a}(0.1 \mathrm{mmol}), \mathbf{2 a}, \mathrm{Na}_{2} \mathrm{CO}_{3}$ in $t$-Butanol $(1 \mathrm{~mL})$ at $80^{\circ} \mathrm{C}$ for $12 \mathrm{~h}$, under $\mathrm{N}_{2} \cdot{ }^{\mathrm{b}}$ The yields were determined by ${ }^{1} \mathrm{H}$ NMR analysis of the crude product using $\mathrm{CH}_{2} \mathrm{Br}_{2}$ as the internal standard. ${ }^{\mathrm{c}}$ Under air atmosphere. 


\section{3. ${ }^{1} \mathrm{H}$ and ${ }^{13} \mathrm{C}\{1 \mathrm{H}\}$ NMR Spectra}

\section{${ }^{1} \mathrm{H}$ NMR (500 MHz, $\mathrm{CDCl}_{3}$ ) of 3a}

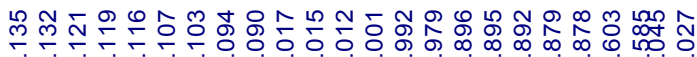

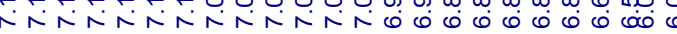
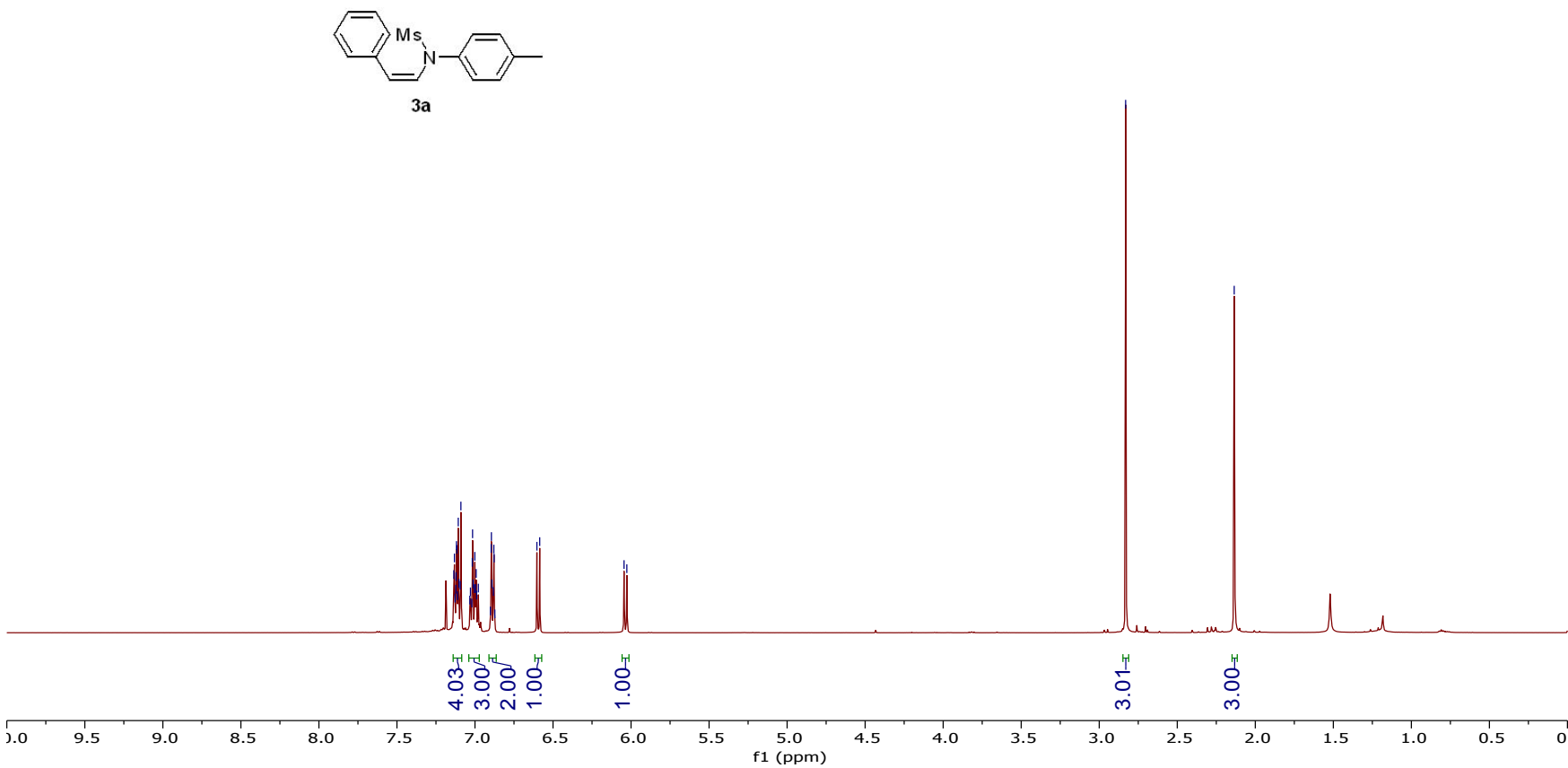

${ }^{13} \mathrm{C}\{1 \mathrm{H}\}$ NMR (126 $\left.\mathrm{MHz}, \mathrm{CDCl}_{3}\right)$ of 3a

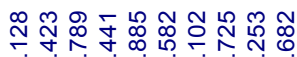

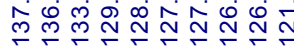

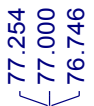

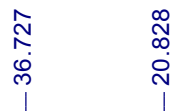
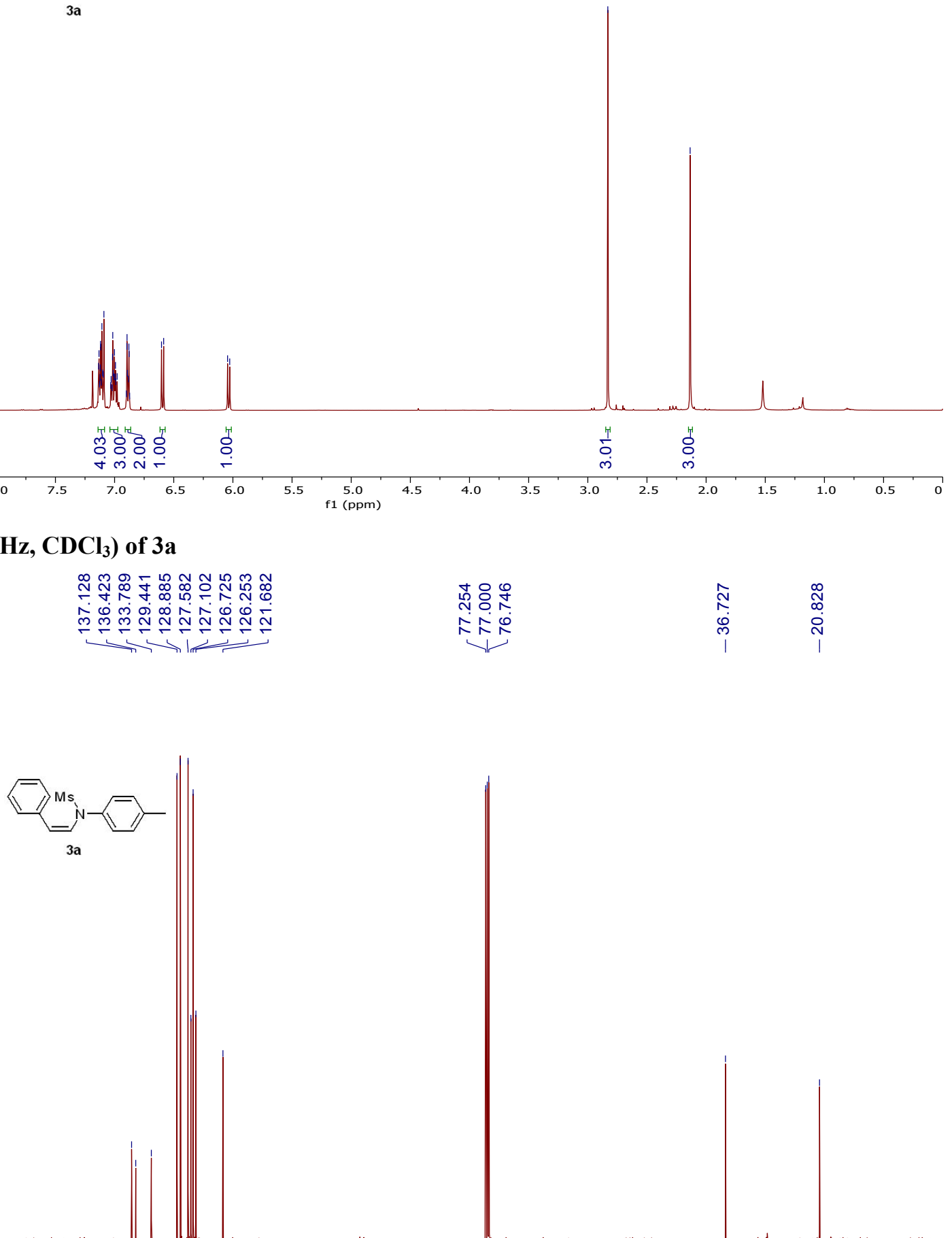
HR-MS spectrum of $3 a$

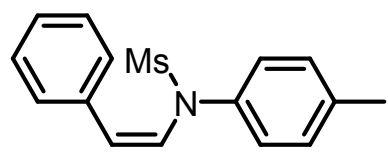

$3 a$

$[\mathrm{M}+\mathrm{H}]^{+}$Cal: 288.1053, Found: 288.1052

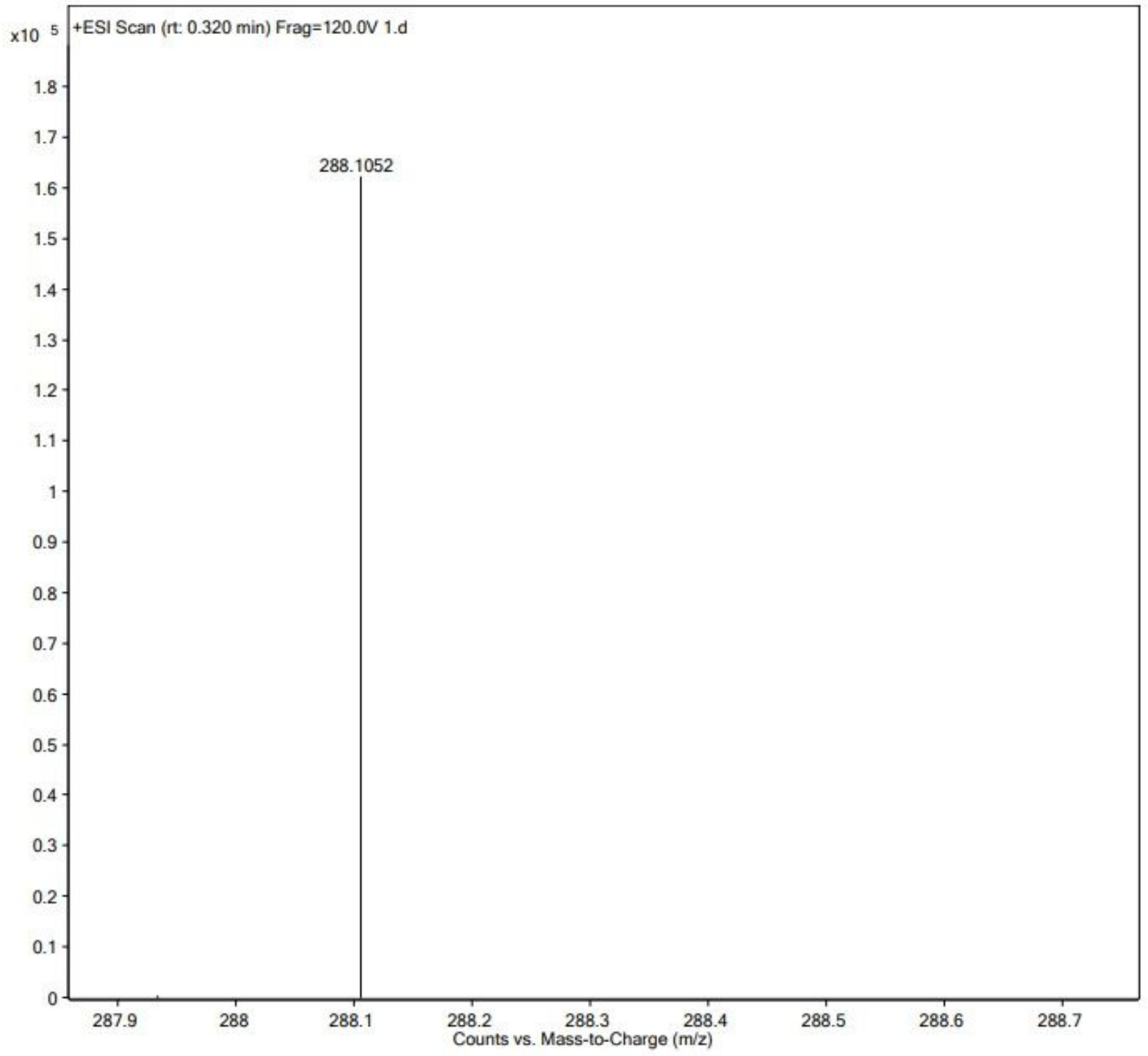



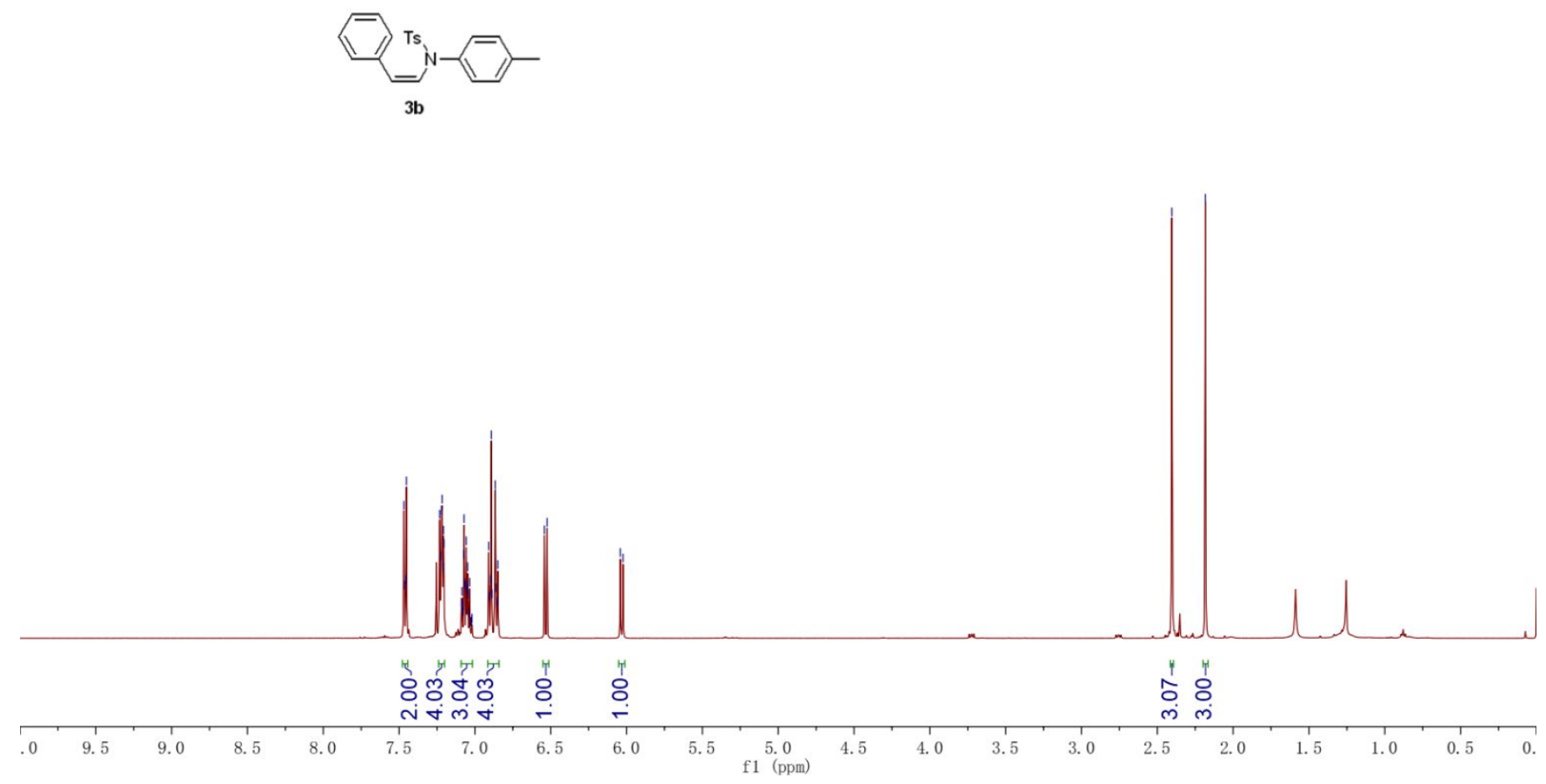

${ }^{13} \mathrm{C}\{1 \mathrm{H}\}$ NMR (126 MHz, $\left.\mathrm{CDCl}_{3}\right)$ of $3 \mathrm{~b}$

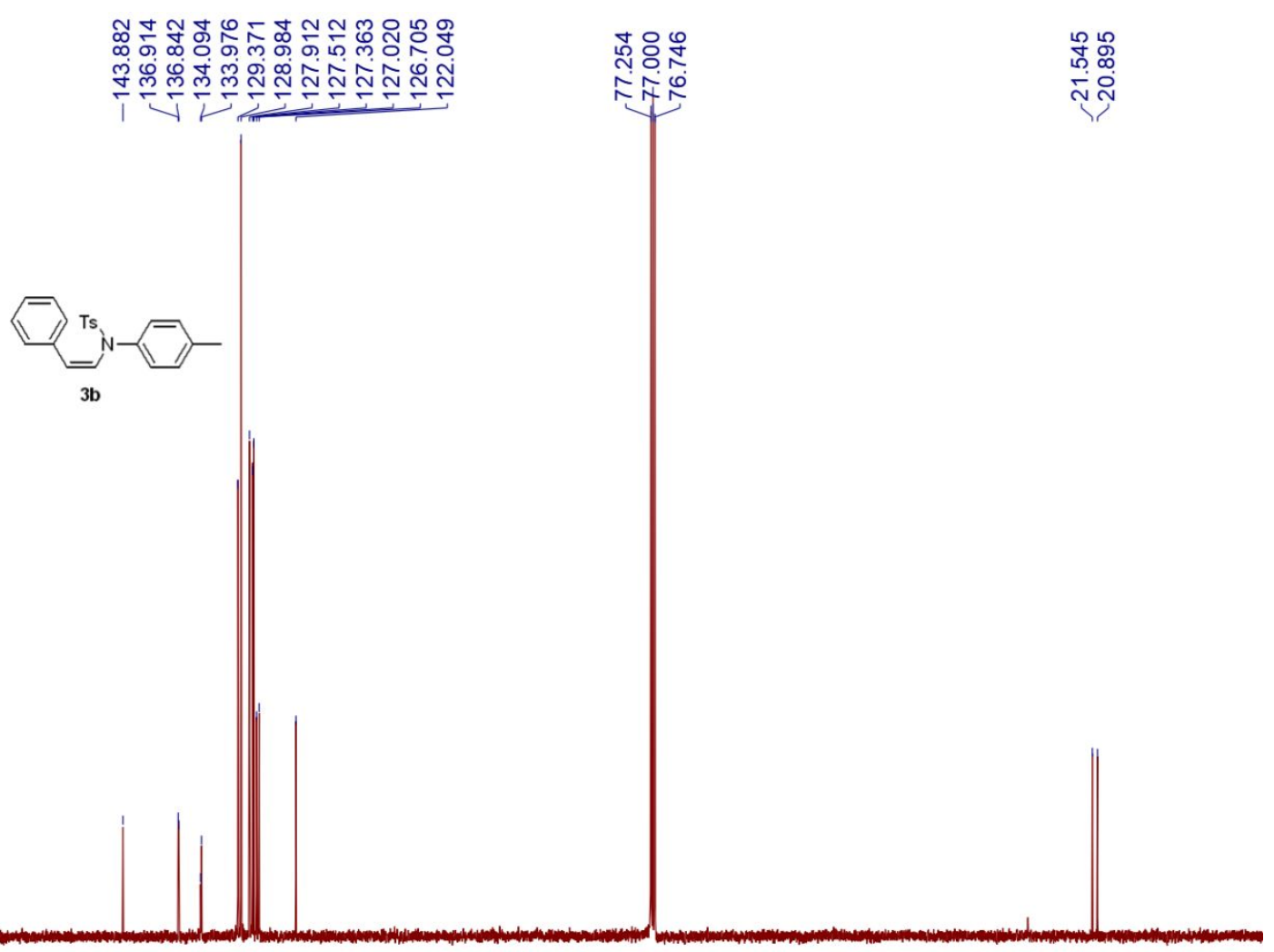


HR-MS spectrum of $3 b$

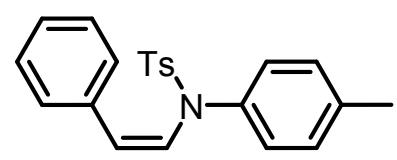

3b

$[\mathrm{M}+\mathrm{H}]^{+}$Cal: 364.1366 , Found: 364.1367

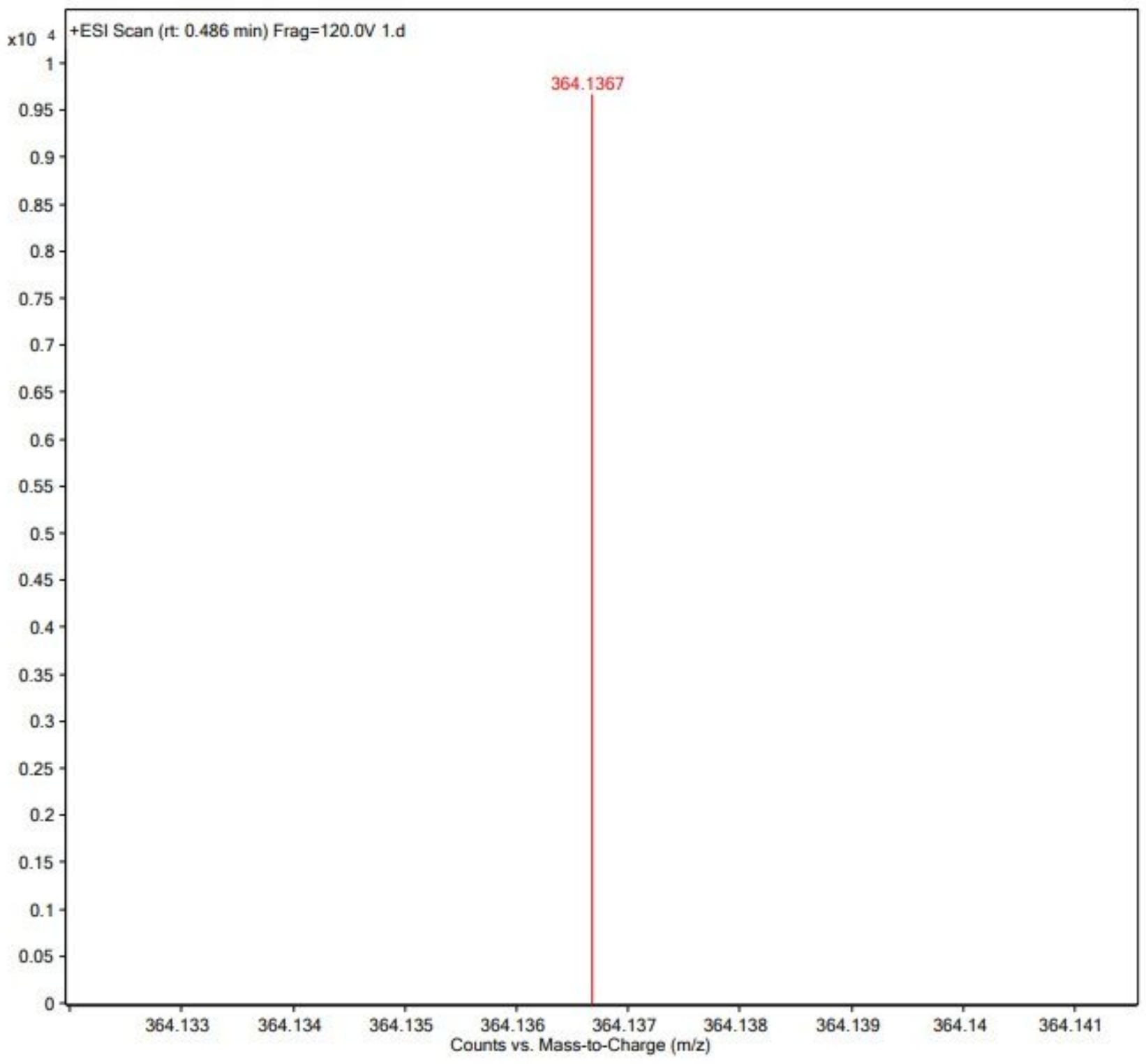




\section{${ }^{1} \mathrm{H}$ NMR (500 MHz, $\left.\mathrm{CDCl}_{3}\right)$ of $3 \mathrm{c}$}

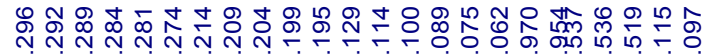

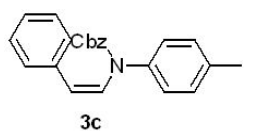

$3 c$

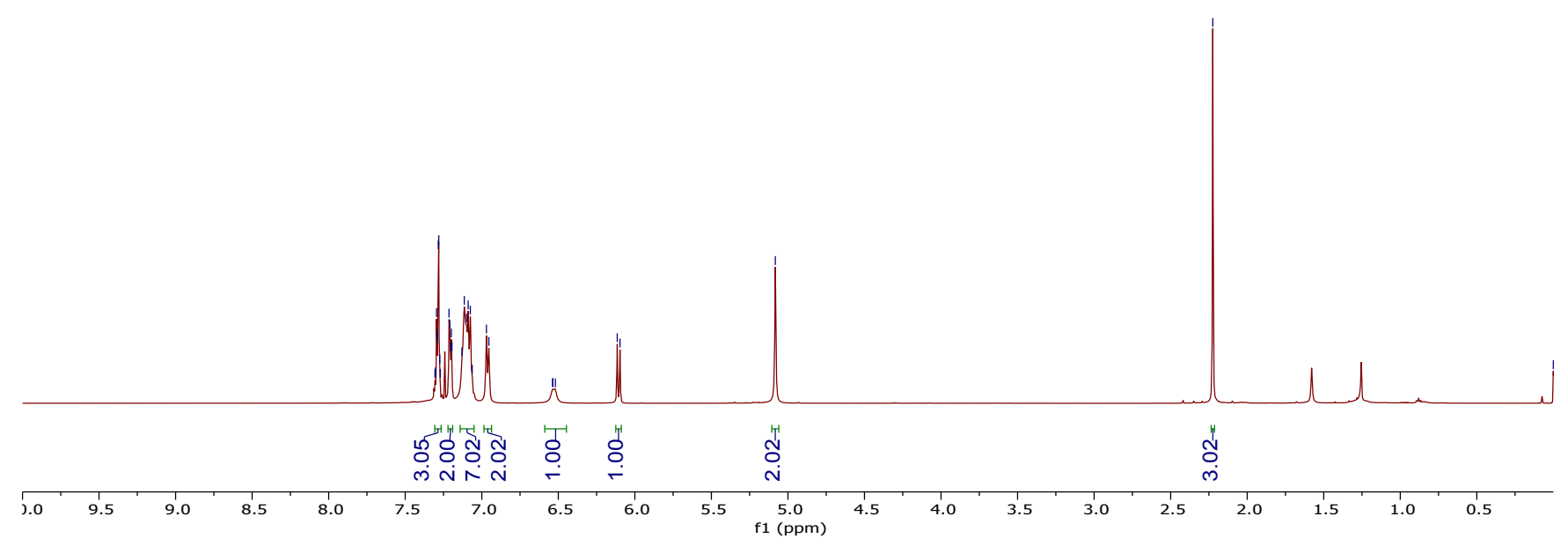

${ }^{13} \mathrm{C}\{1 \mathrm{H}\}$ NMR (126 MHz, $\left.\mathrm{CDCl}_{3}\right)$ of $3 \mathrm{c}$ 


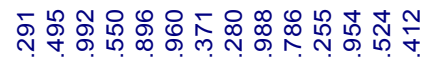

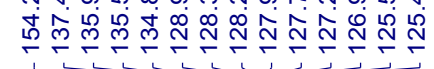

ํㅐㅇํํำ

人र造

11

$\hat{N}$
$\infty$
$\stackrel{N}{0}$
1

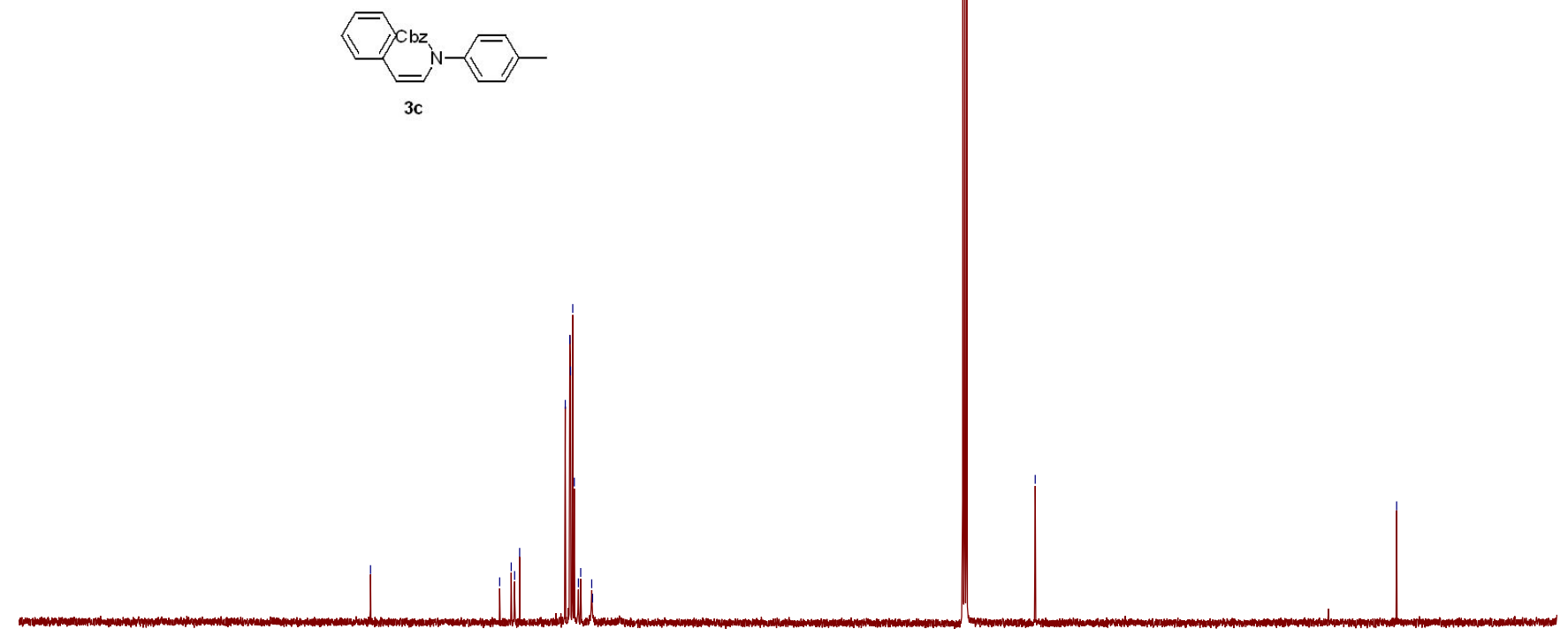

$3 c$

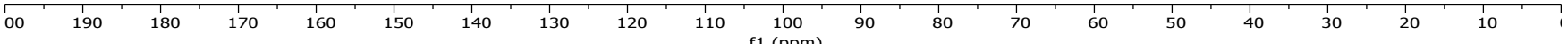

HR-MS spectrum of 3c

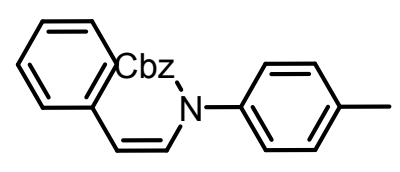

$3 c$

$[\mathrm{M}+\mathrm{H}]^{+}$Cal: 344.1645, Found: 344.1643 


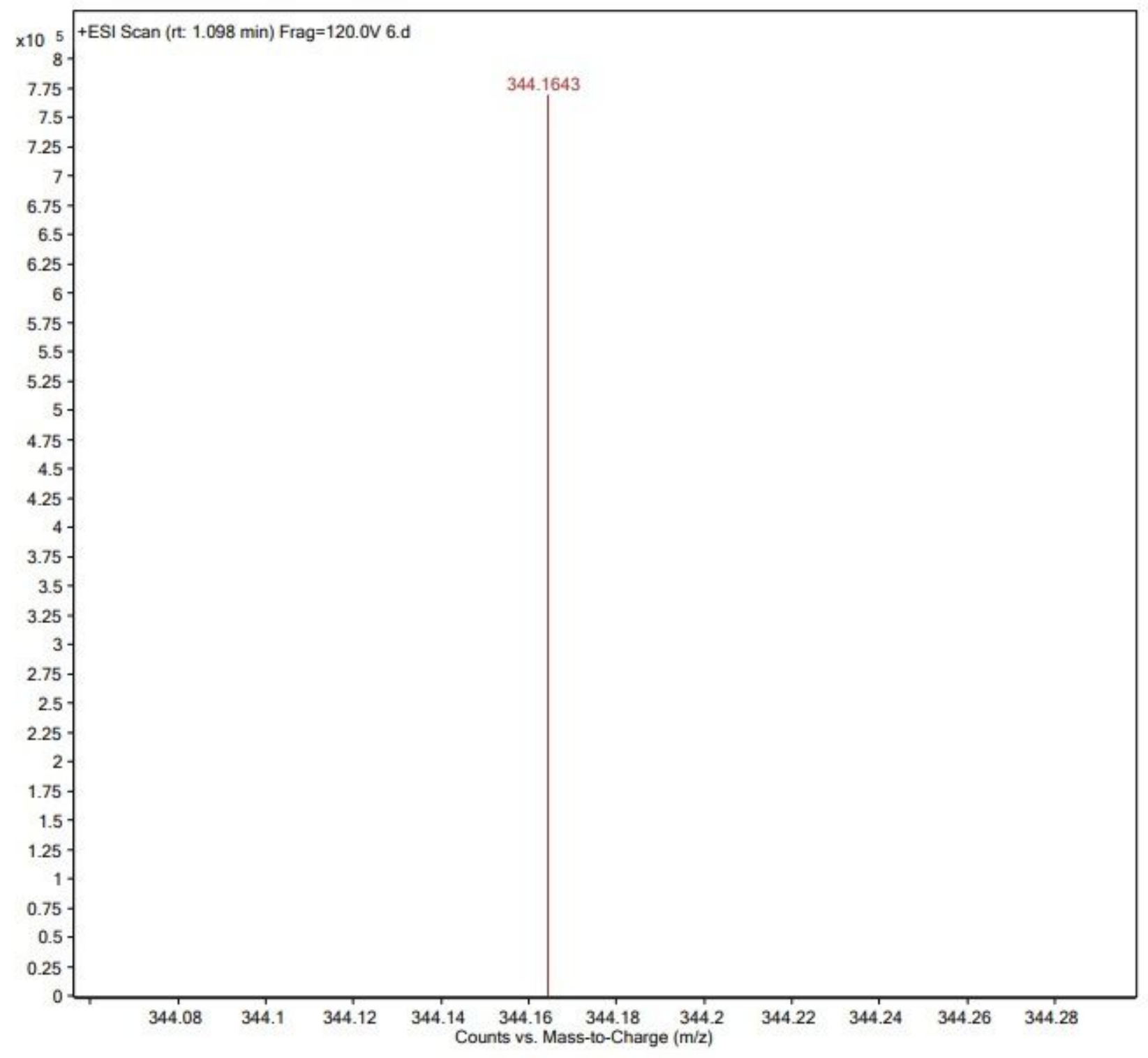

${ }^{1} \mathrm{H}$ NMR (500 MHz, $\mathrm{CDCl}_{3}$ ) of 3d 

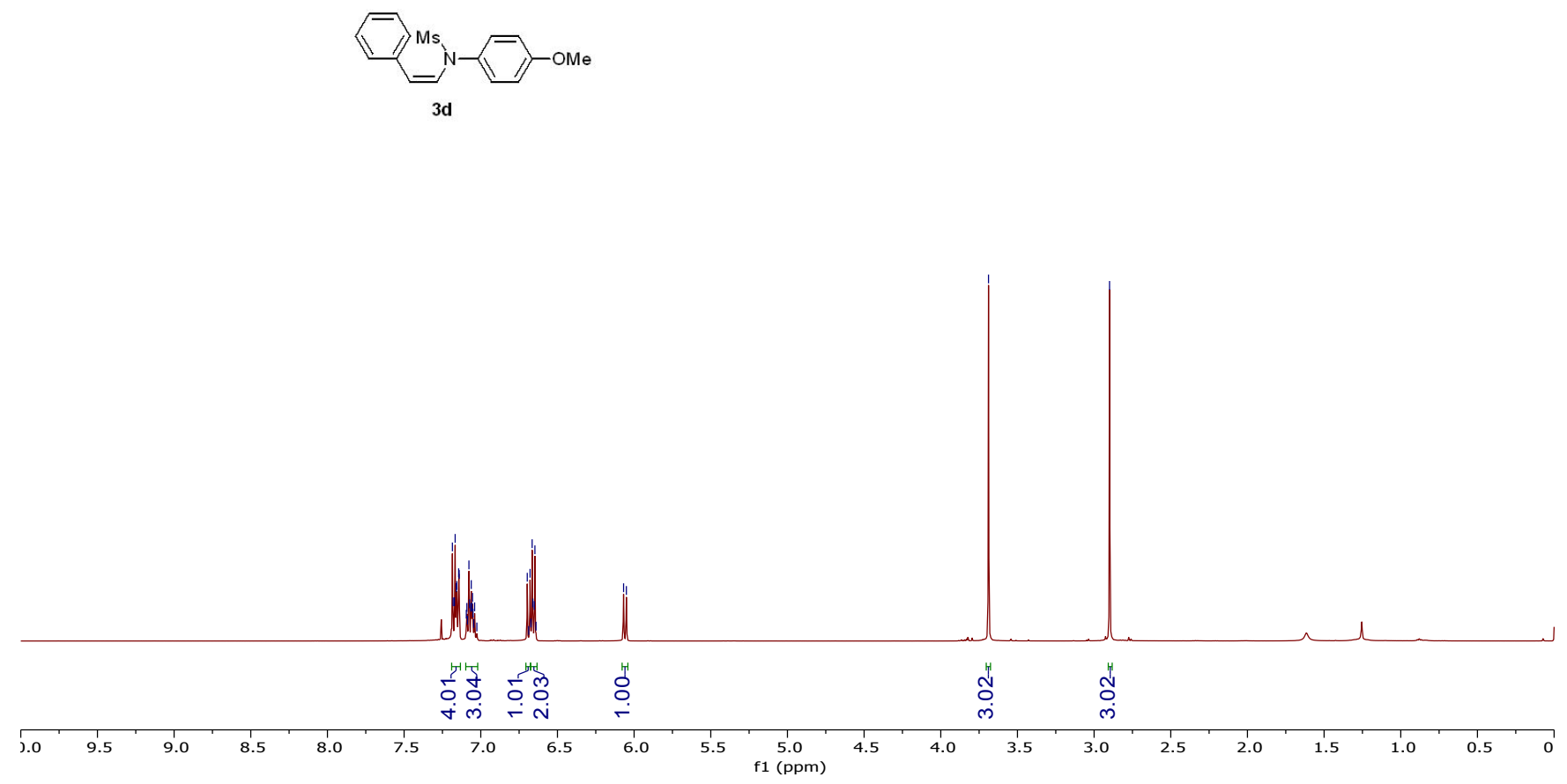

${ }^{13} \mathrm{C}\{1 \mathrm{H}\}$ NMR (126 MHz, $\left.\mathrm{CDCl}_{3}\right)$ of 3d

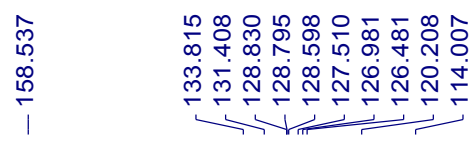

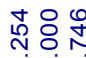

令兑

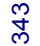

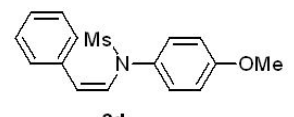

$3 d$
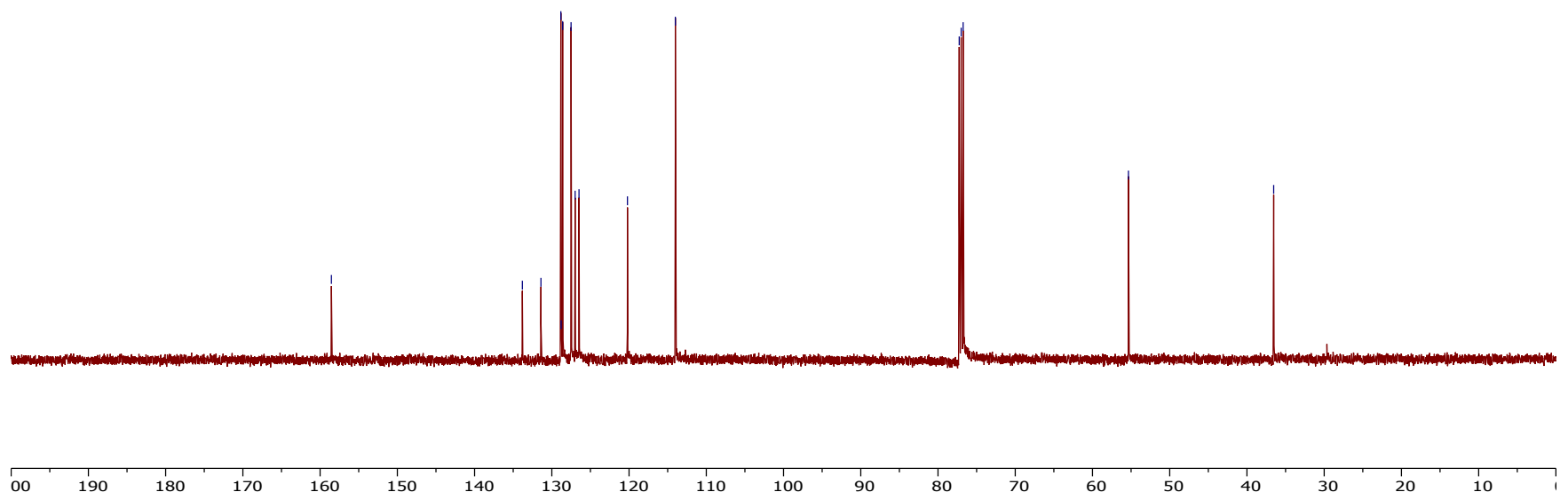

160

$150 \quad 140$

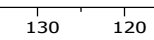

110

$\stackrel{100}{1(\mathrm{ppm})}$

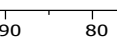

$70 \quad 60$

HR-MS spectrum of $3 d$ 


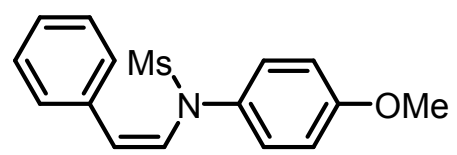

$3 d$

$[\mathrm{M}+\mathrm{H}]^{+}$Cal: 304.1002, Found: 304.1003

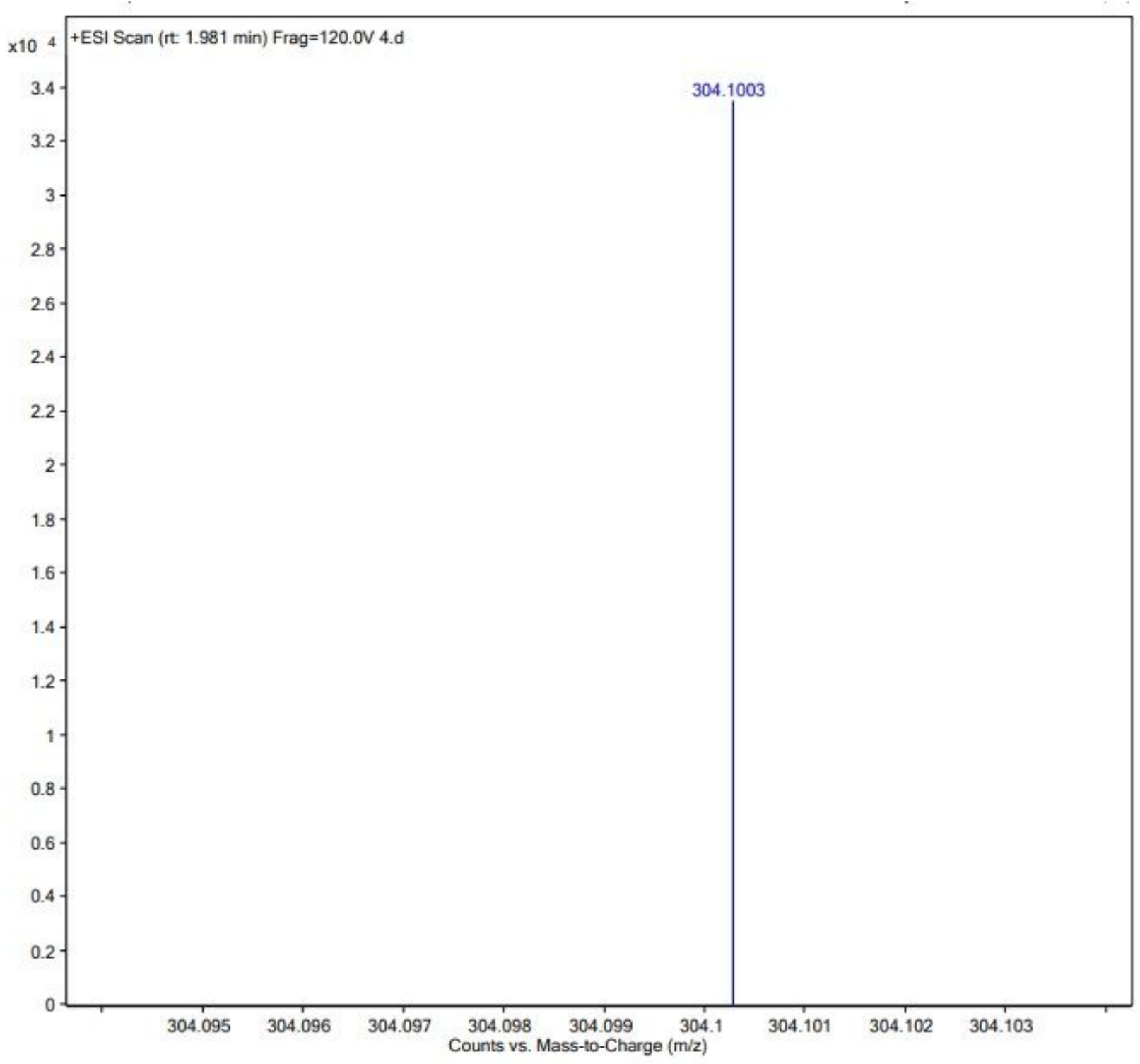

${ }^{1} \mathrm{H}$ NMR (500 MHz, $\mathrm{CDCl}_{3}$ ) of $3 \mathrm{e}$ 

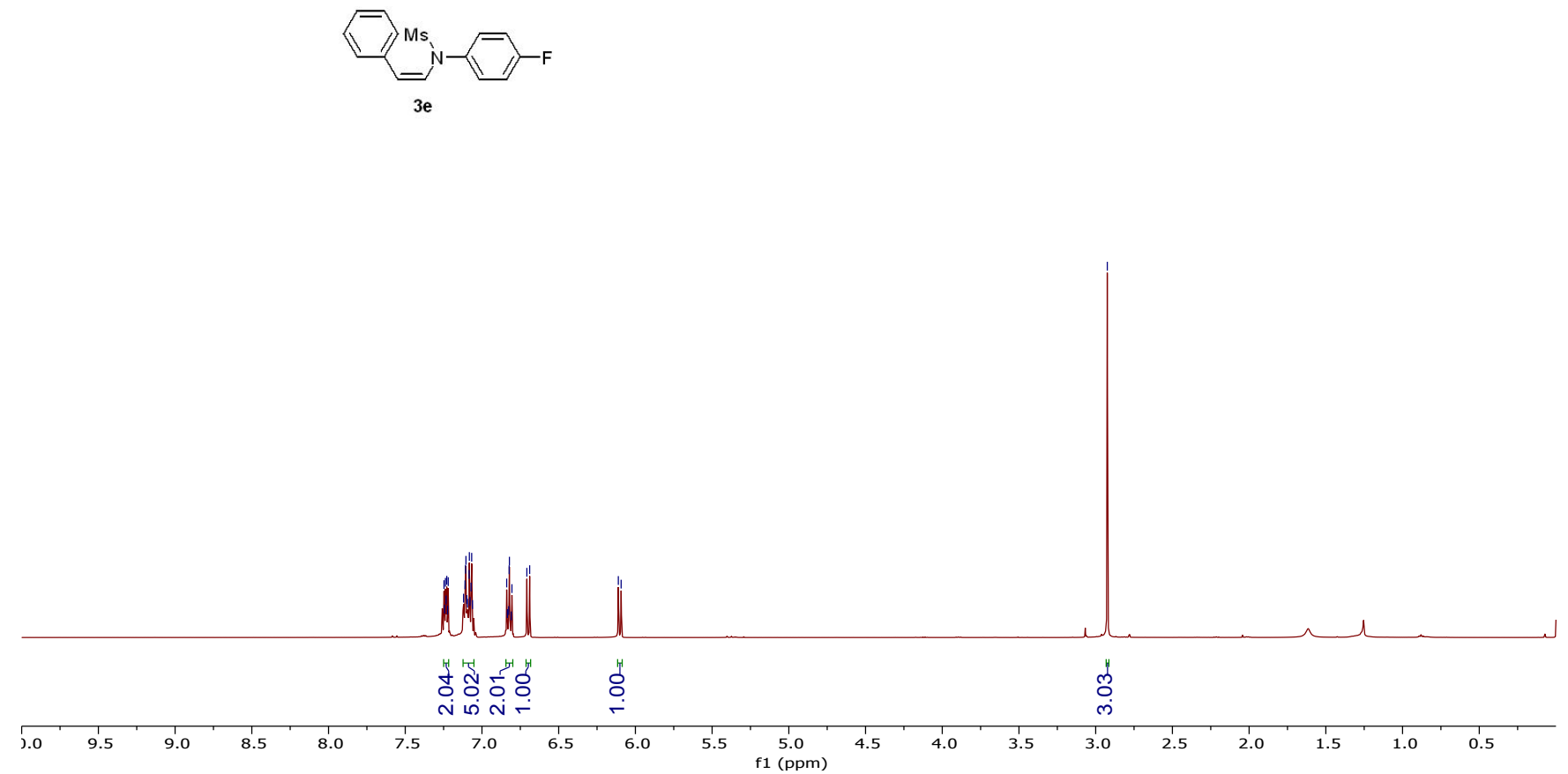

${ }^{13} \mathrm{C}\{1 \mathrm{H}\}$ NMR (126 MHz, $\left.\mathrm{CDCl}_{3}\right)$ of $3 \mathrm{e}$
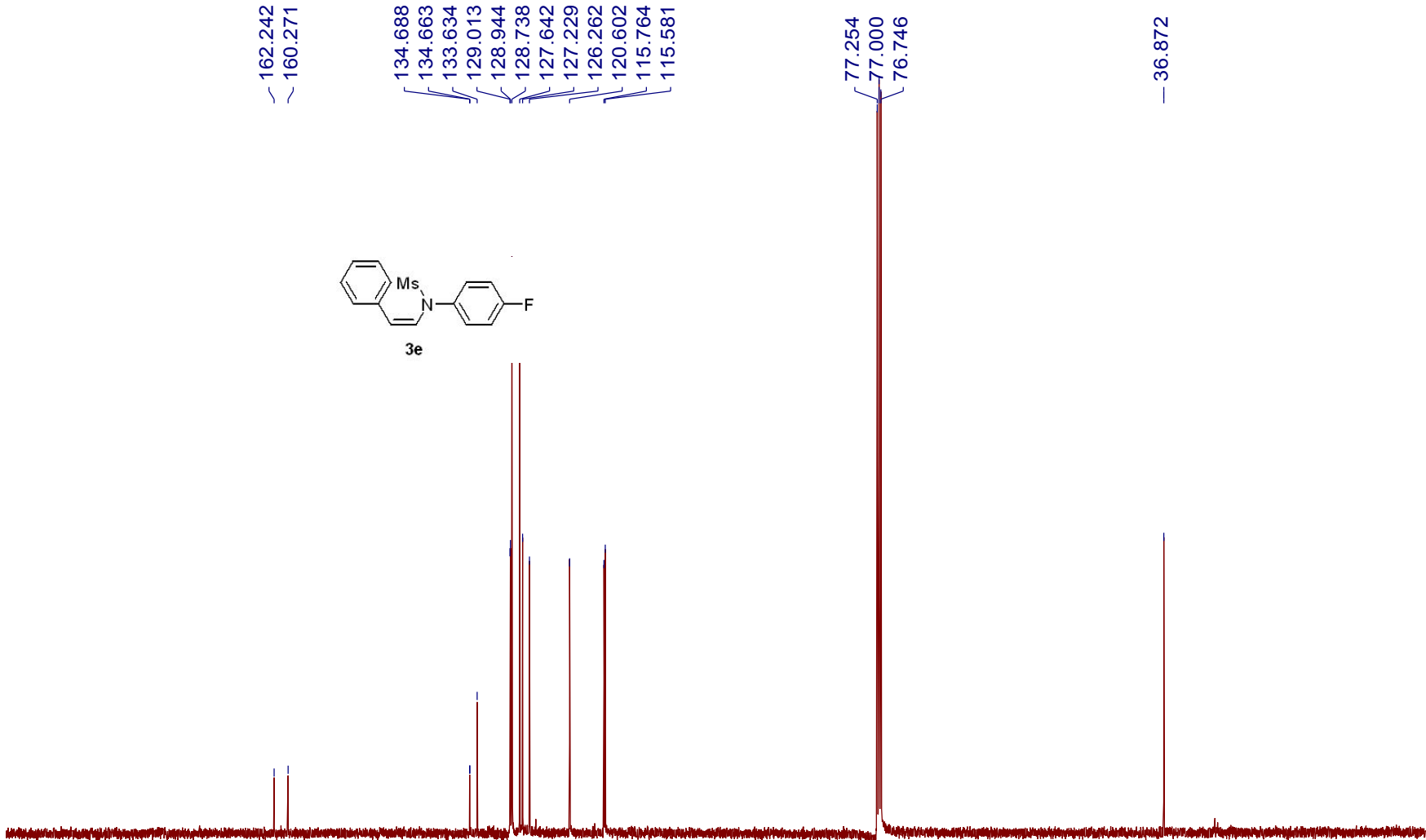

3e 


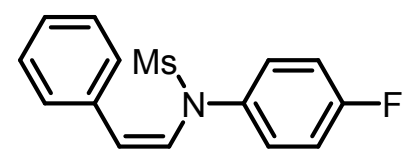

$3 e$

$[\mathrm{M}+\mathrm{H}]^{+}$Cal: 292.0802, Found: 292.0802

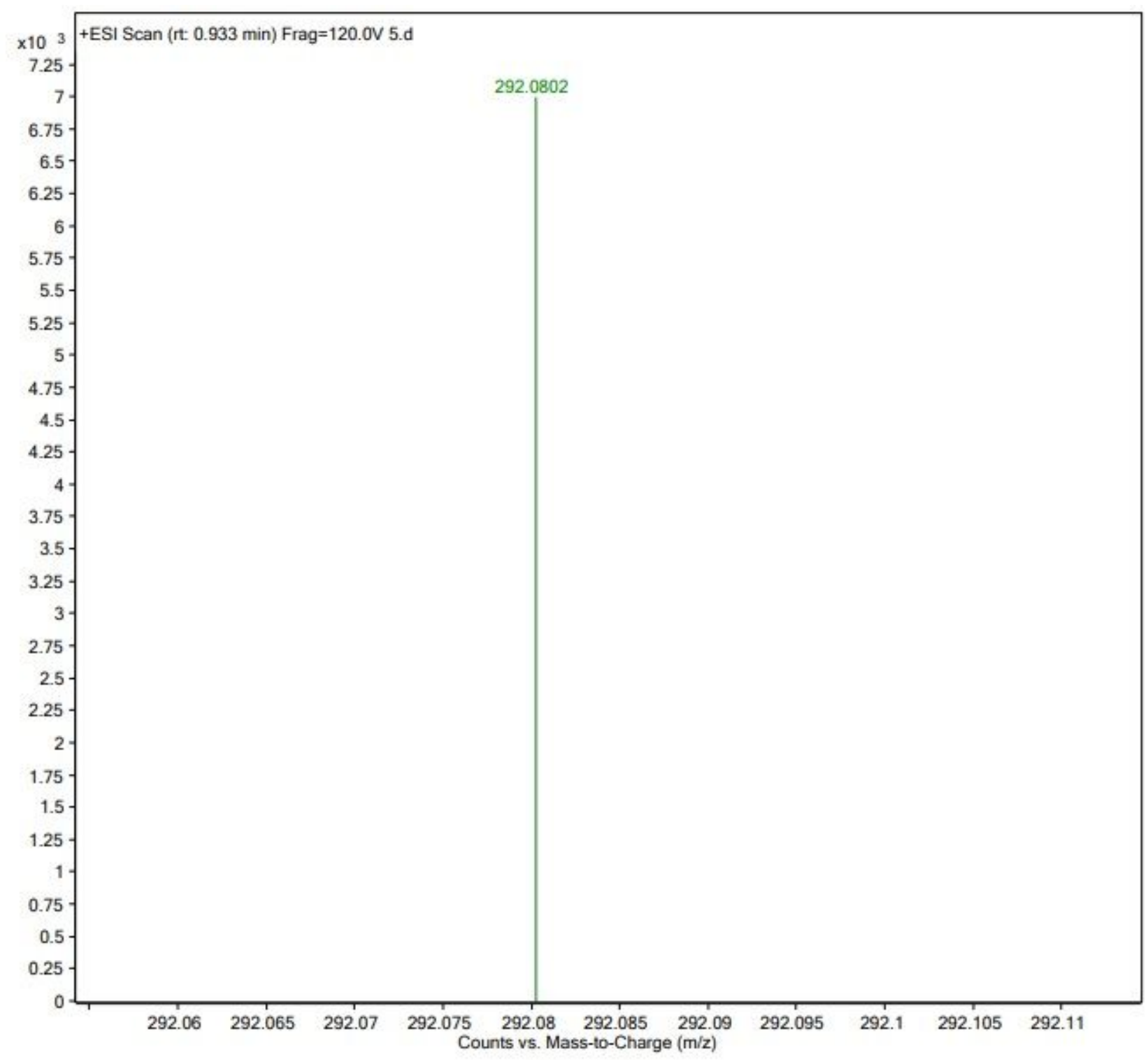



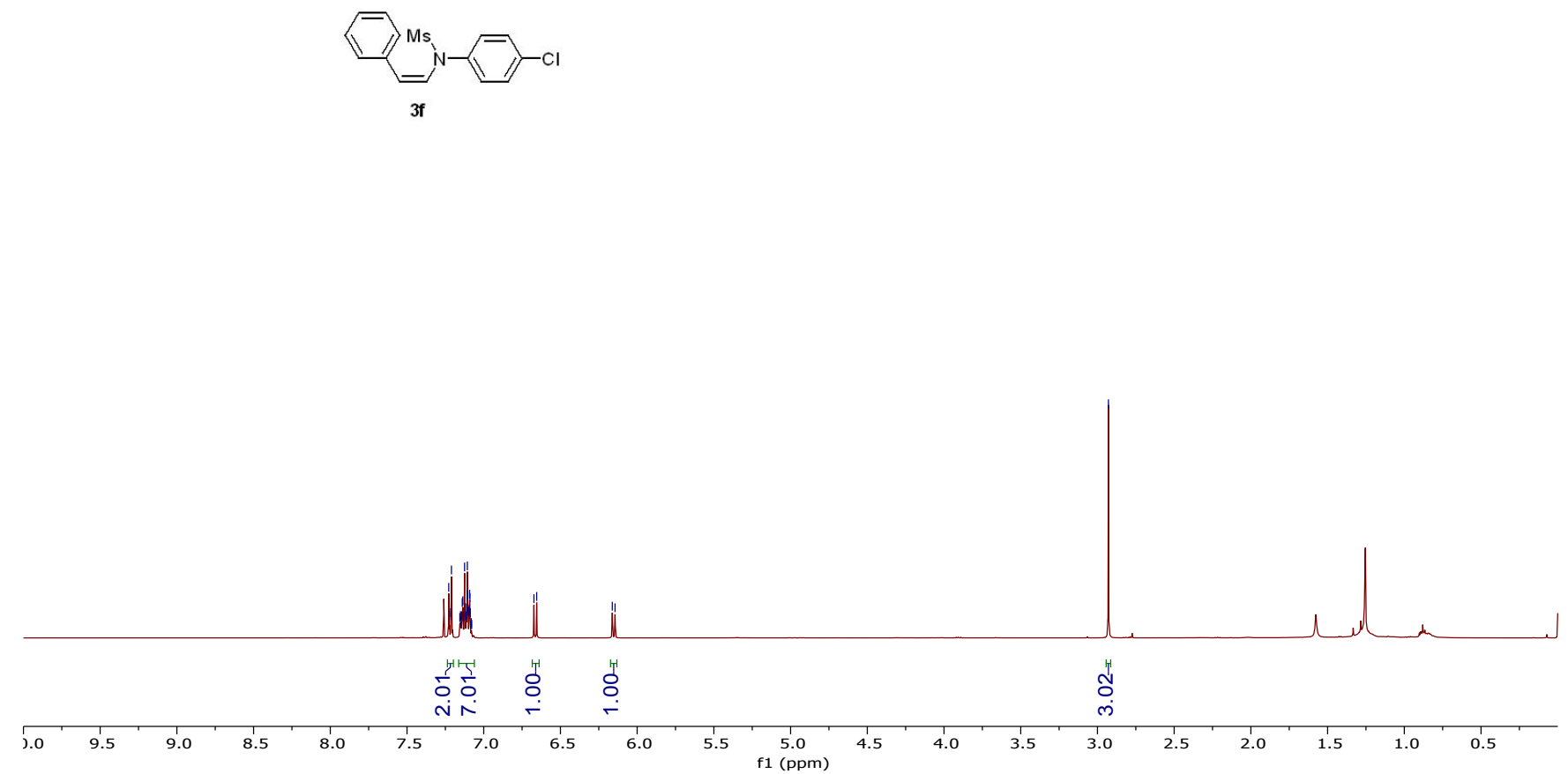

${ }^{13} \mathrm{C}\{1 \mathrm{H}\}$ NMR (126 MHz, $\left.\mathrm{CDCl}_{3}\right)$ of $3 \mathrm{f}$
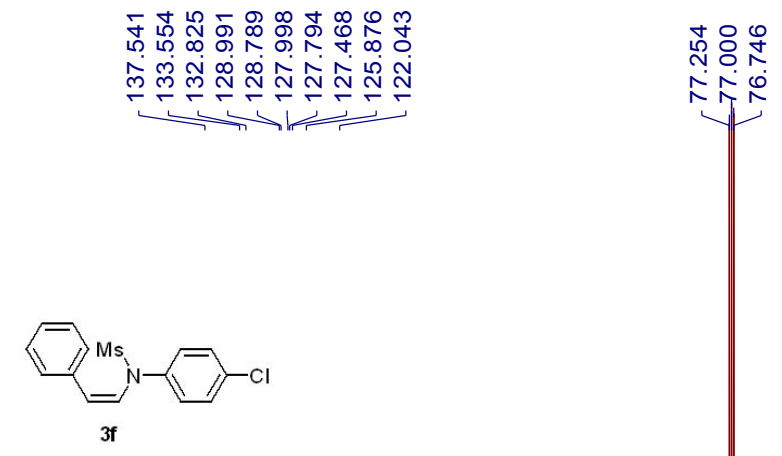

$\begin{array}{cc}0 & 0 \\ 0 & 0 \\ & 0 \\ & \mathbb{2} \\ 1 & 1\end{array}$

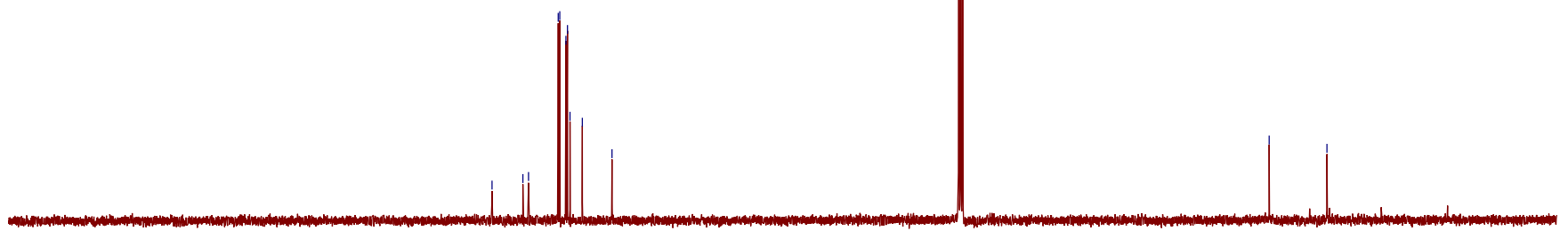

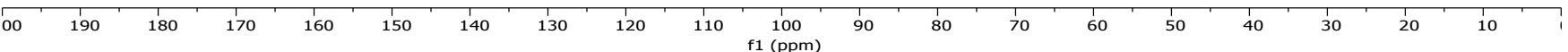

HR-MS spectrum of $3 f$ 


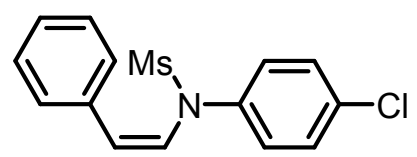

$3 f$

$[\mathrm{M}+\mathrm{H}]^{+}$Cal: 308.0507, Found: 308.0506

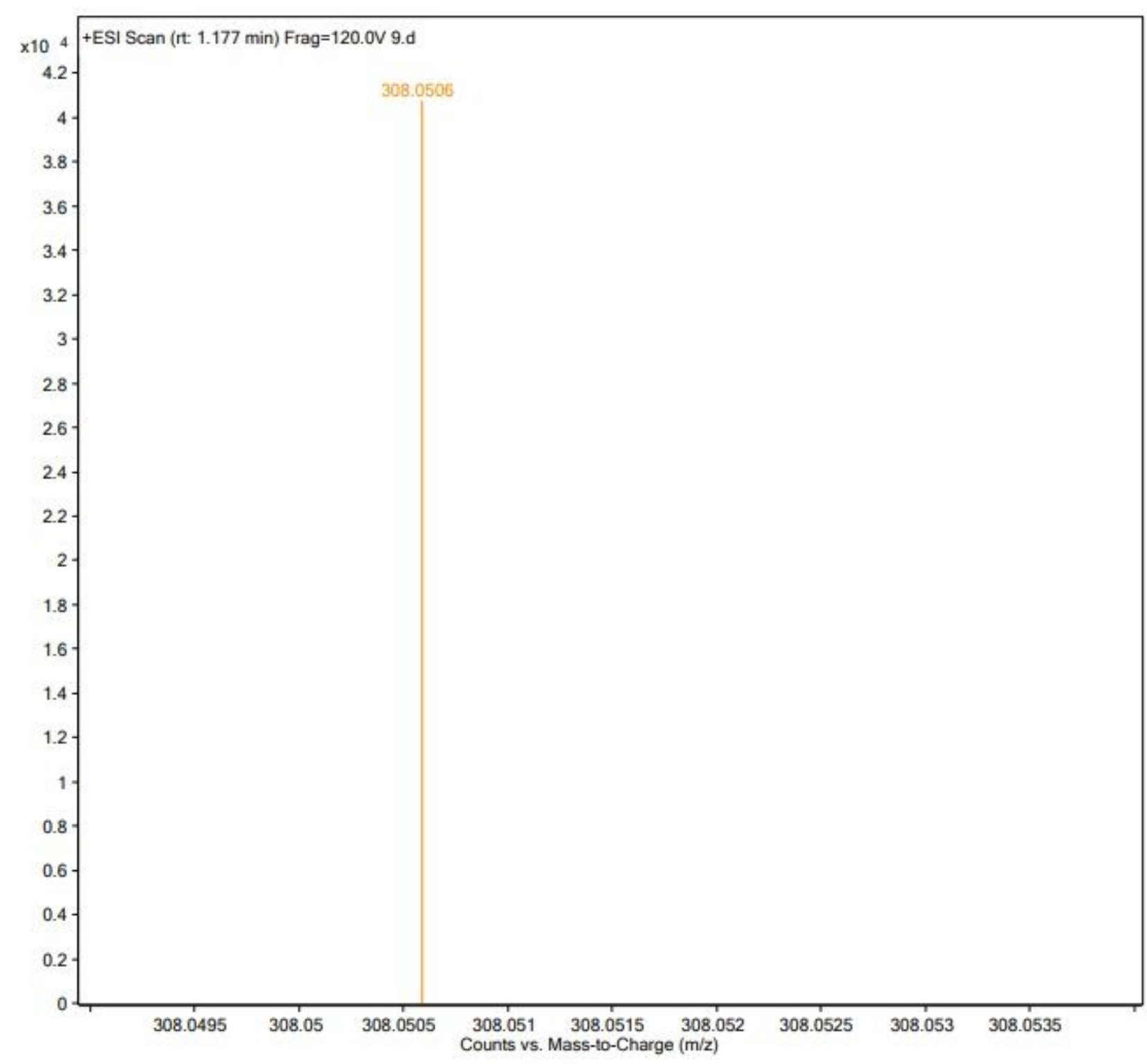

${ }^{1} \mathrm{H}$ NMR (500 MHz, $\mathrm{CDCl}_{3}$ ) of $3 \mathrm{~g}$ 


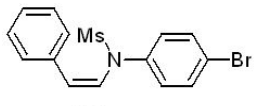

3g

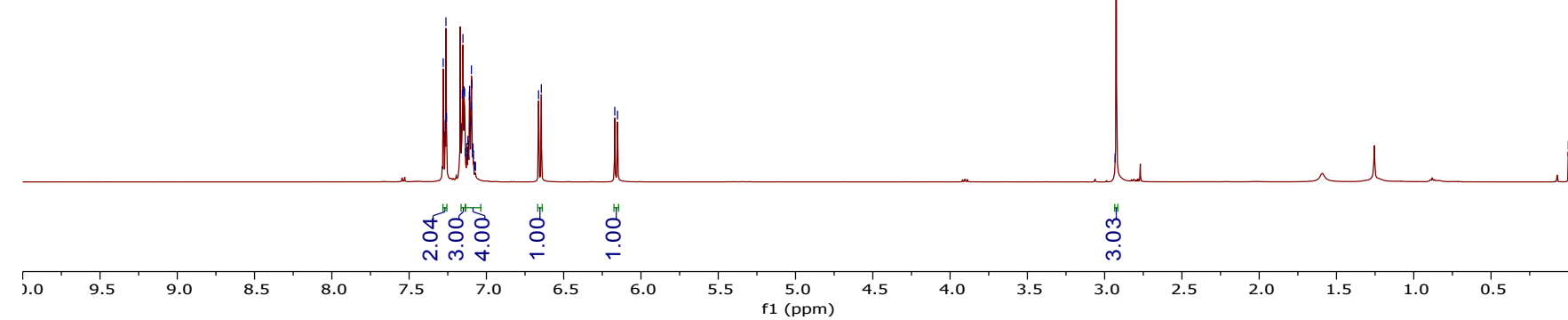

\section{${ }^{13} \mathrm{C}\{1 \mathrm{H}\}$ NMR (126 MHz, $\left.\mathrm{CDCl}_{3}\right)$ of $3 \mathrm{~g}$}

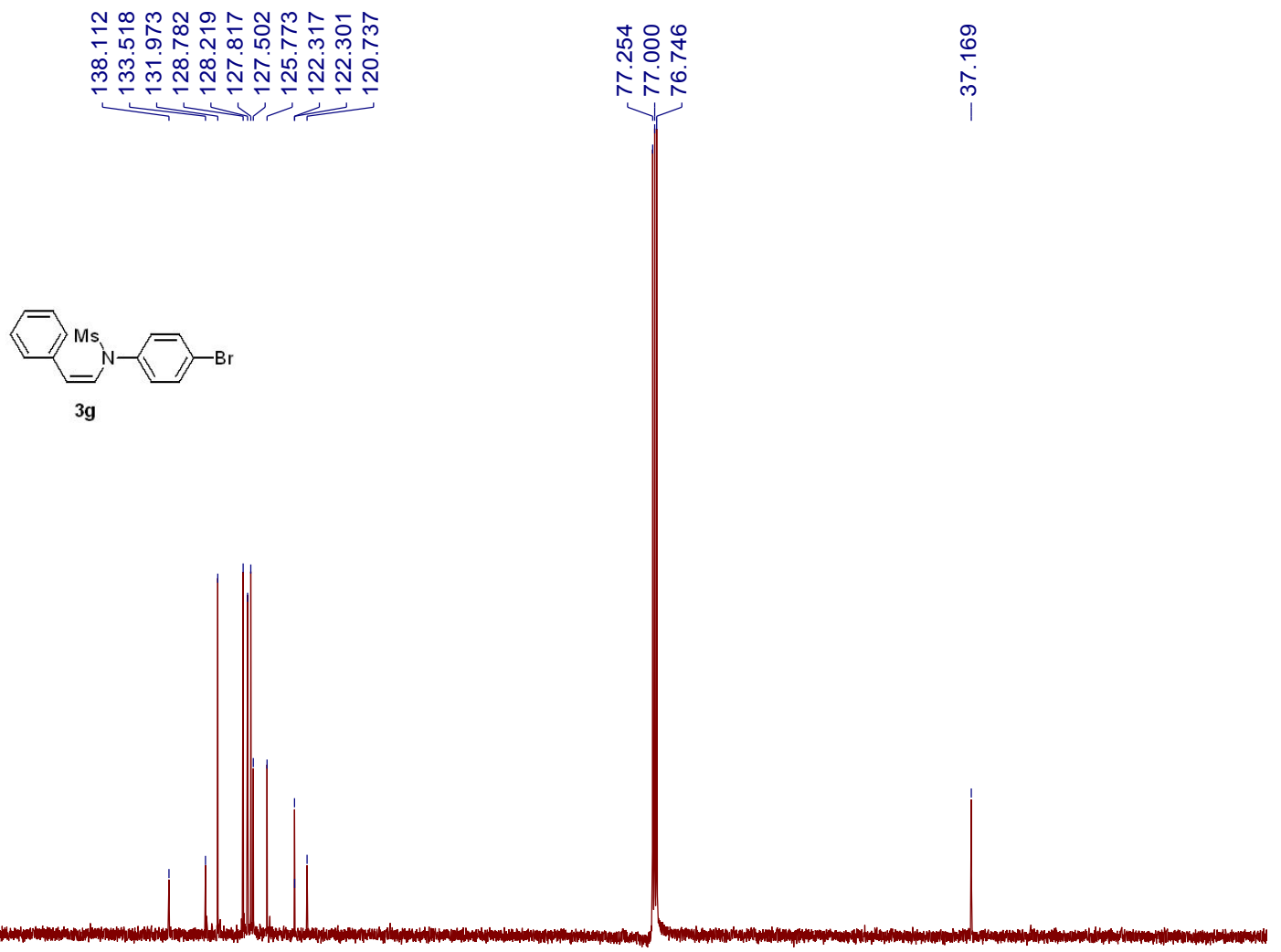




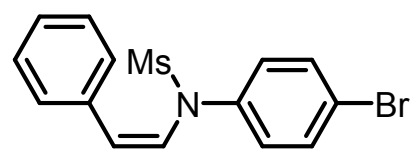

$3 \mathrm{~g}$

$[\mathrm{M}+\mathrm{H}]^{+}$Cal: 352.0001, Found: 352.0002

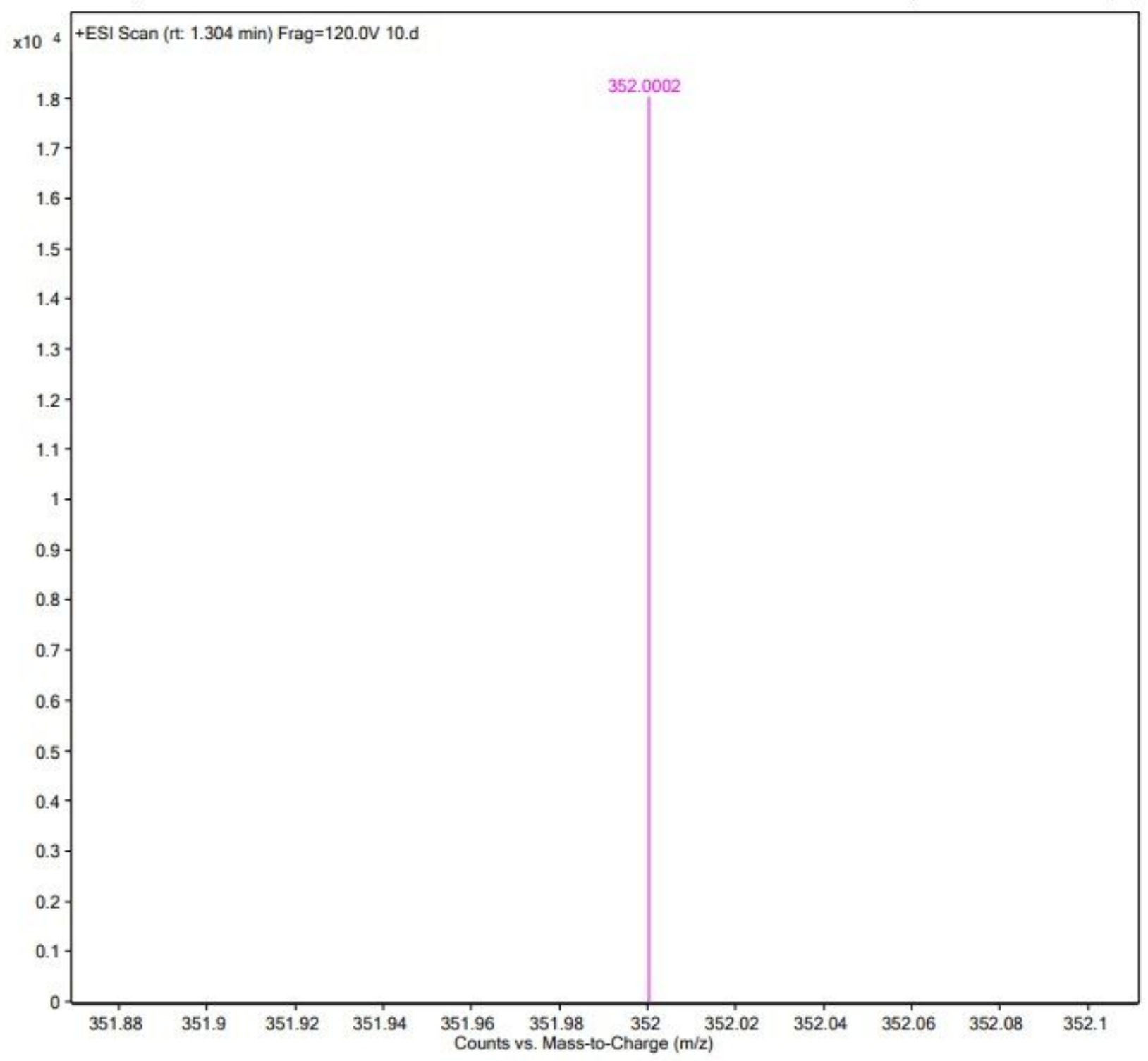




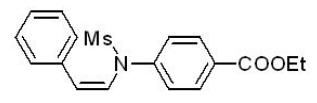

$3 \mathrm{~h}$

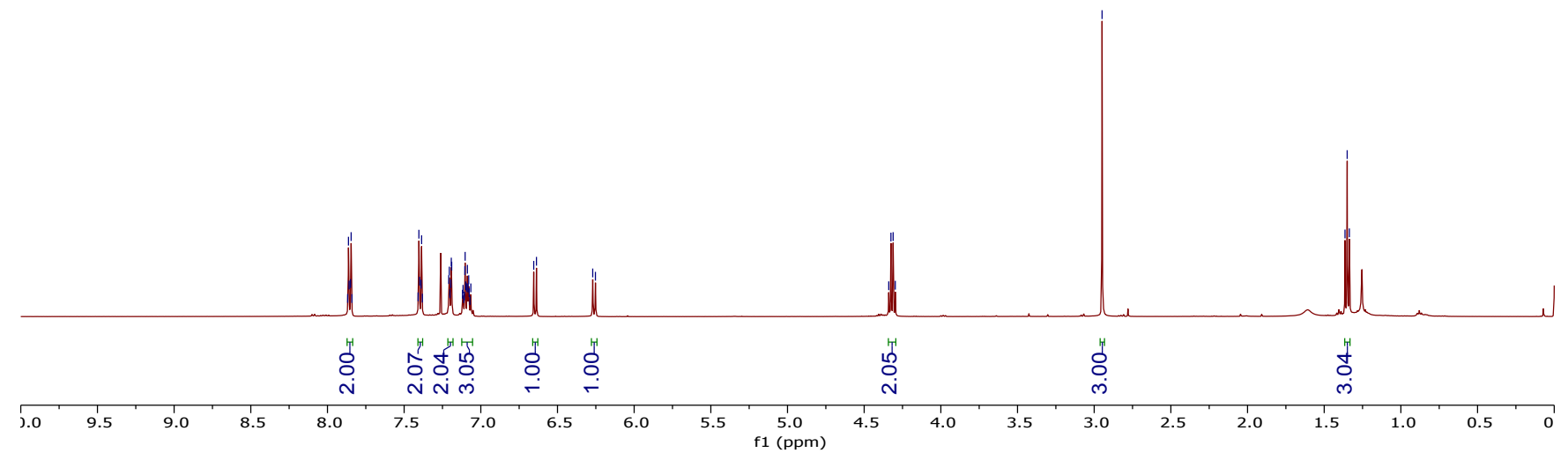

${ }^{13} \mathrm{C}\{1 \mathrm{H}\}$ NMR (126 MHz, $\left.\mathrm{CDCl}_{3}\right)$ of $3 \mathrm{~h}$
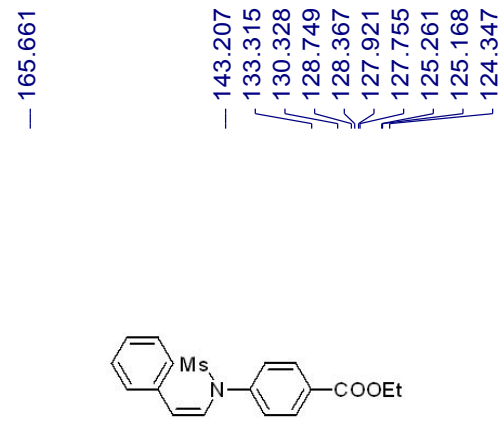

$3 \mathbf{h}$

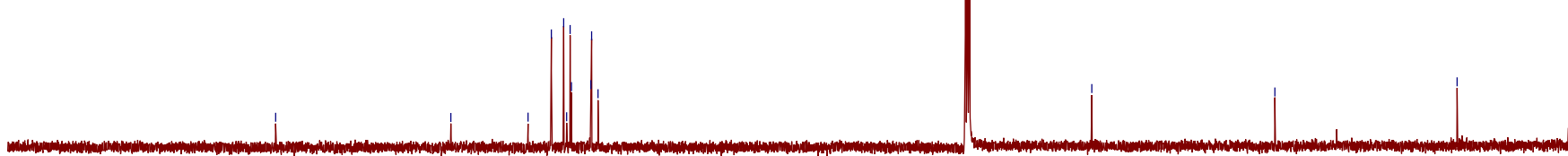




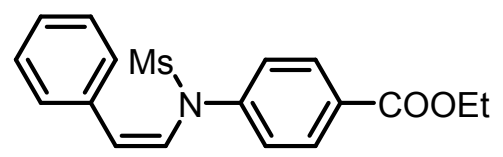

$3 \mathrm{~h}$

$[\mathrm{M}+\mathrm{H}]^{+}$Cal: 346.1108, Found: 346.1107

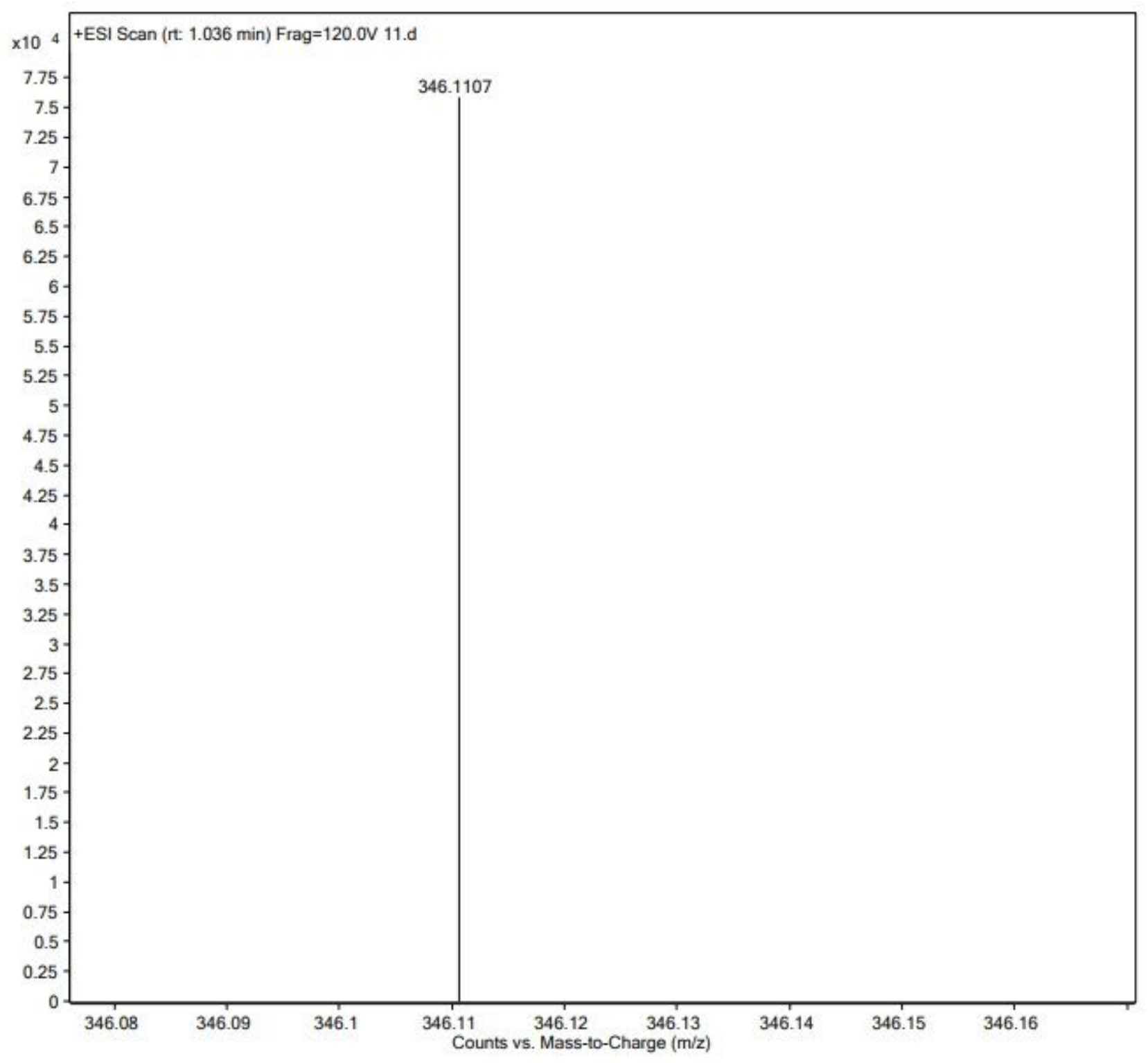




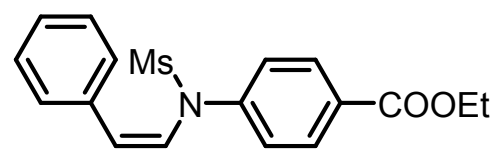

$3 \mathrm{~h}$

$[\mathrm{M}+\mathrm{H}]^{+}$Cal: 288.1053, Found: 288.1051

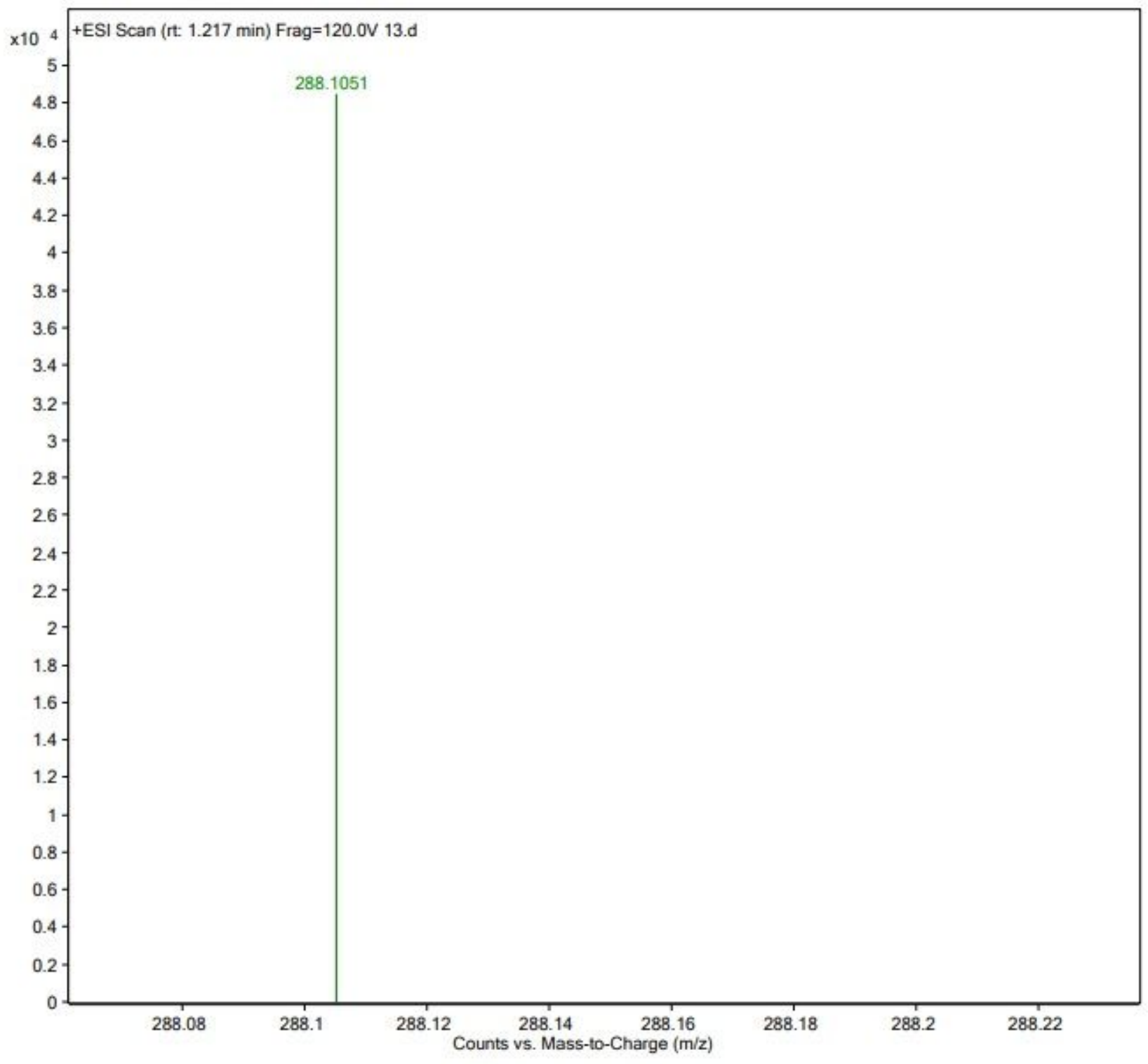




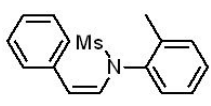

3j

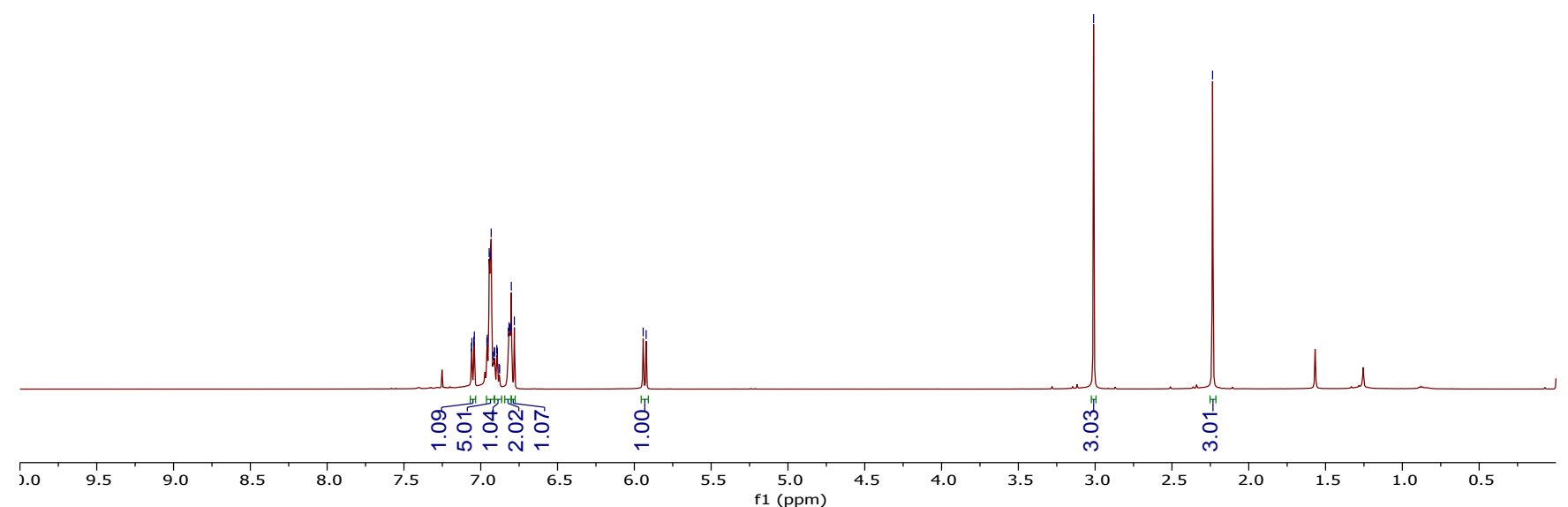

${ }^{13} \mathrm{C}\{1 \mathrm{H}\}$ NMR (126 $\left.\mathrm{MHz}, \mathrm{CDCl}_{3}\right)$ of $3 \mathrm{j}$

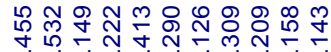

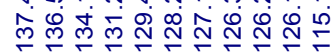

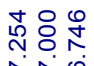

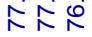

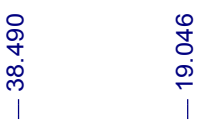

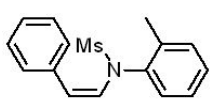

3j

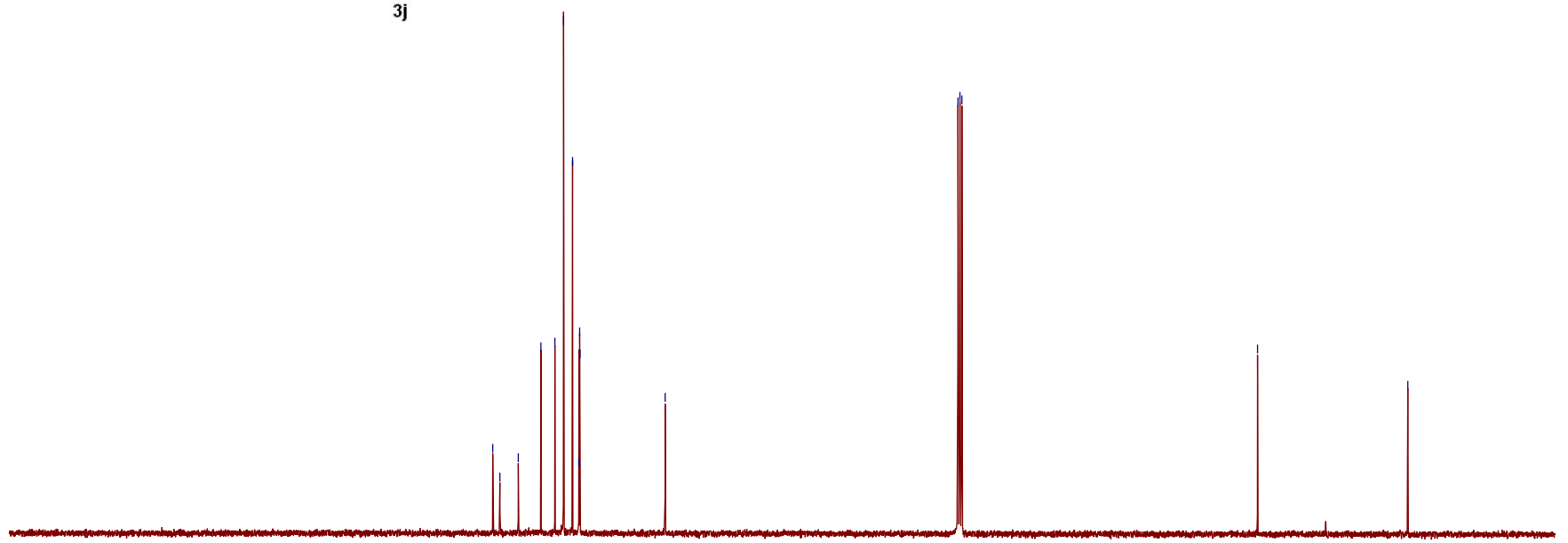

$00 \quad 190 \quad 180 \quad 170 \quad 160$

$150 \quad 140$

$130 \quad 120$

$10 \begin{gathered}100 \\ \text { f1 }(\mathrm{ppm})\end{gathered}$

80

70

$\begin{array}{llllll}1 & 1 & 1 & 1 & 1 & 1 \\ 10 & 40 & 30 & 20 & 10\end{array}$

HR-MS spectrum of $\mathbf{3 j}$ 


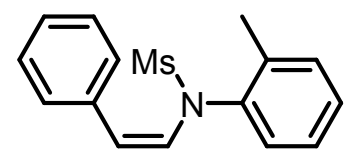

3j

$[\mathrm{M}+\mathrm{H}]^{+}$Cal: 288.1053, Found: 288.1050

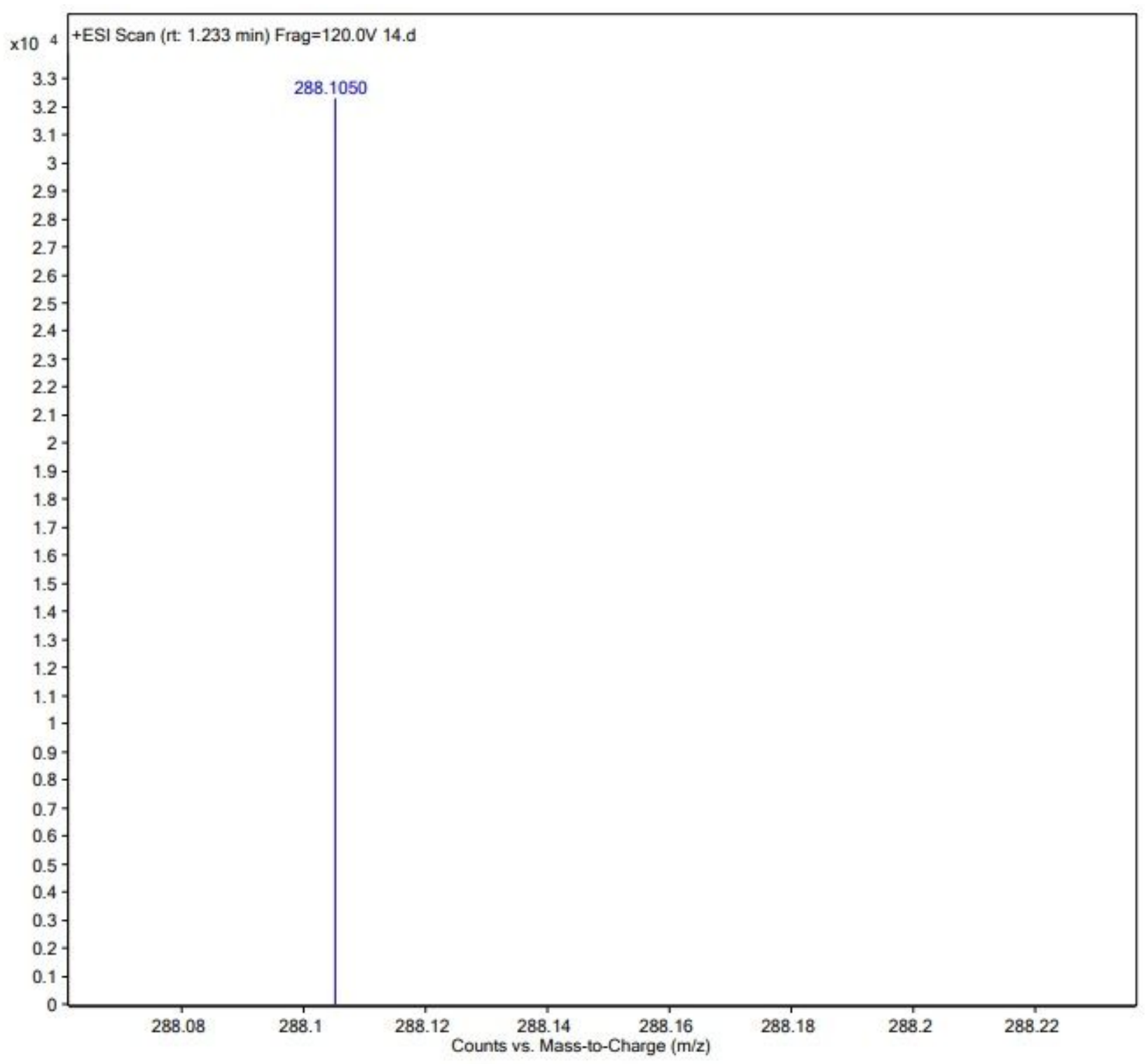




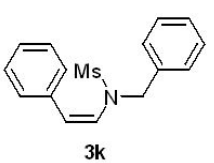

3k

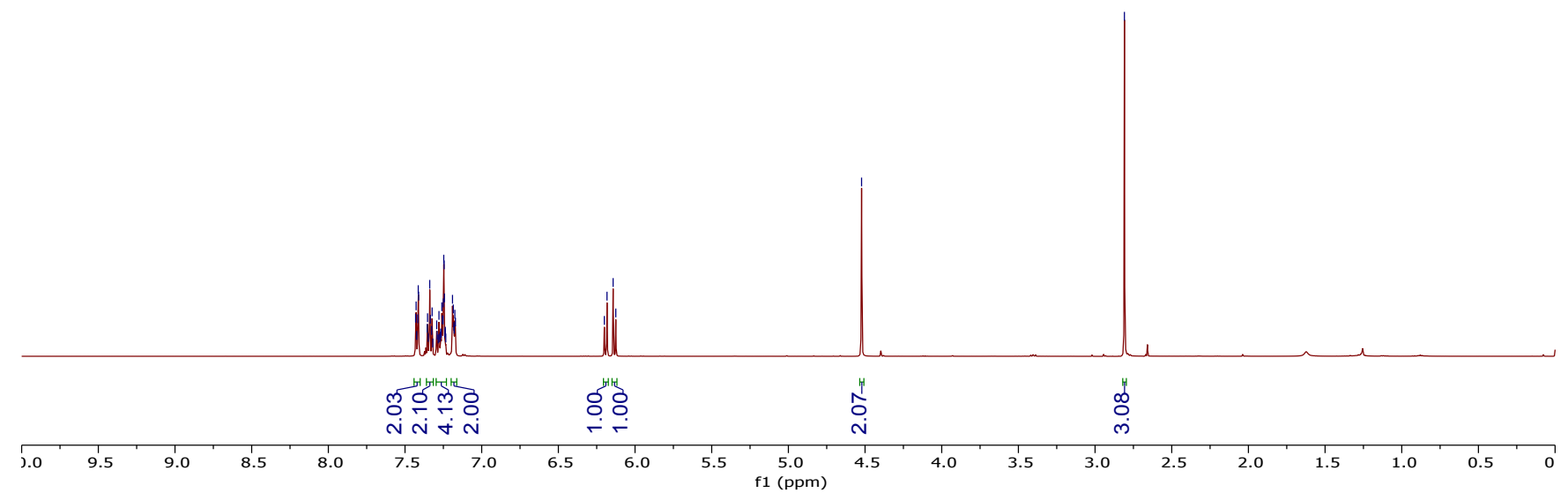

${ }^{13} \mathrm{C}\{1 \mathrm{H}\}$ NMR (126 MHz, $\left.\mathrm{CDCl}_{3}\right)$ of $3 \mathrm{k}$

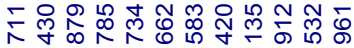

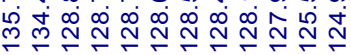

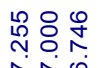

ㅅํㅇ

$\begin{array}{ll}\stackrel{n}{m} & \circ \\ & 0 \\ \text { in } & 0 \\ 1 & 1\end{array}$

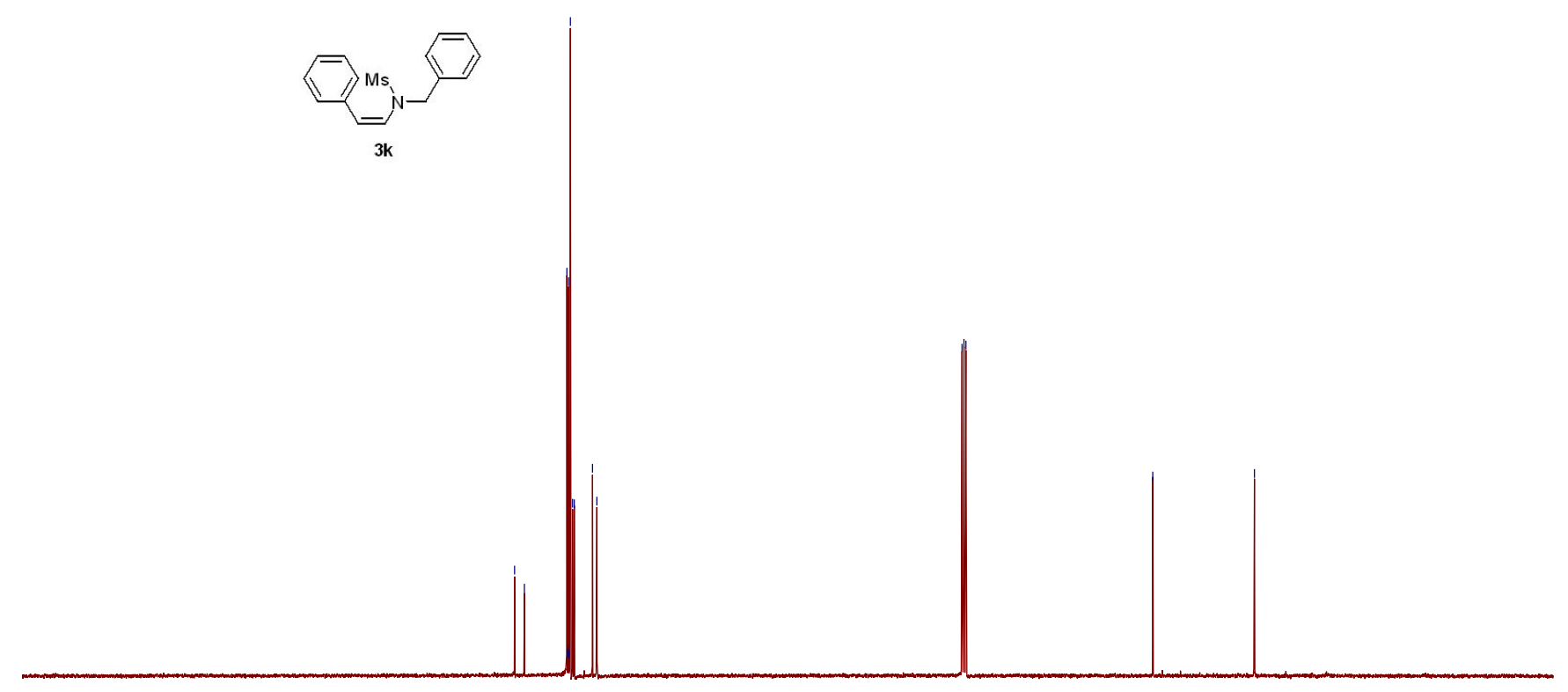

3k

.

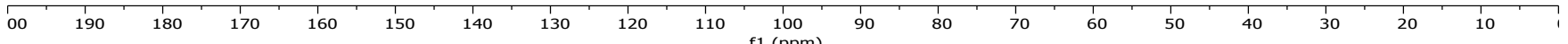

HR-MS spectrum of $3 \mathrm{k}$ 


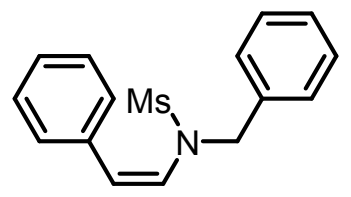

3k

$[\mathrm{M}+\mathrm{H}]^{+}$Cal: 280.1053, Found: 288.1053

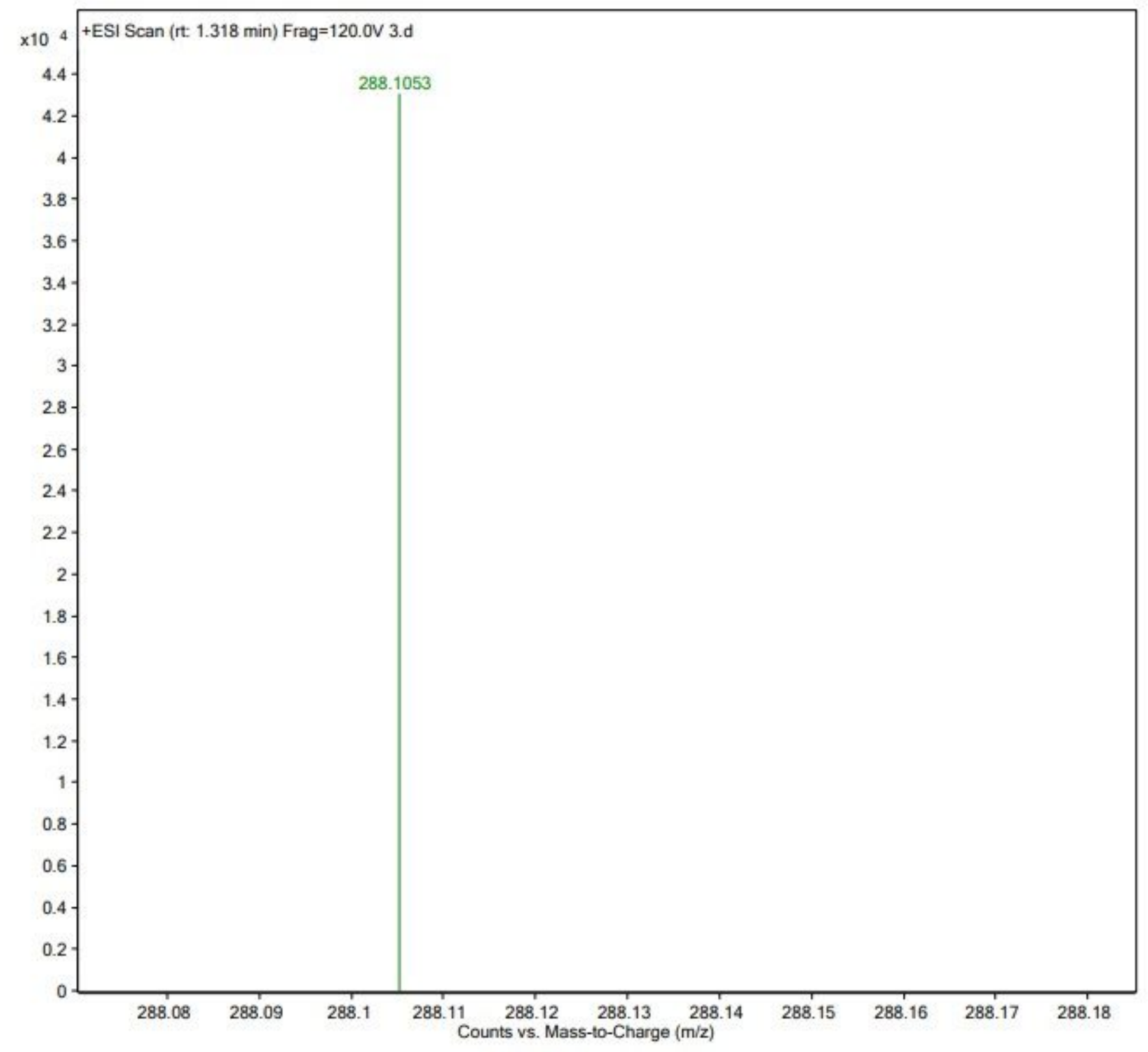



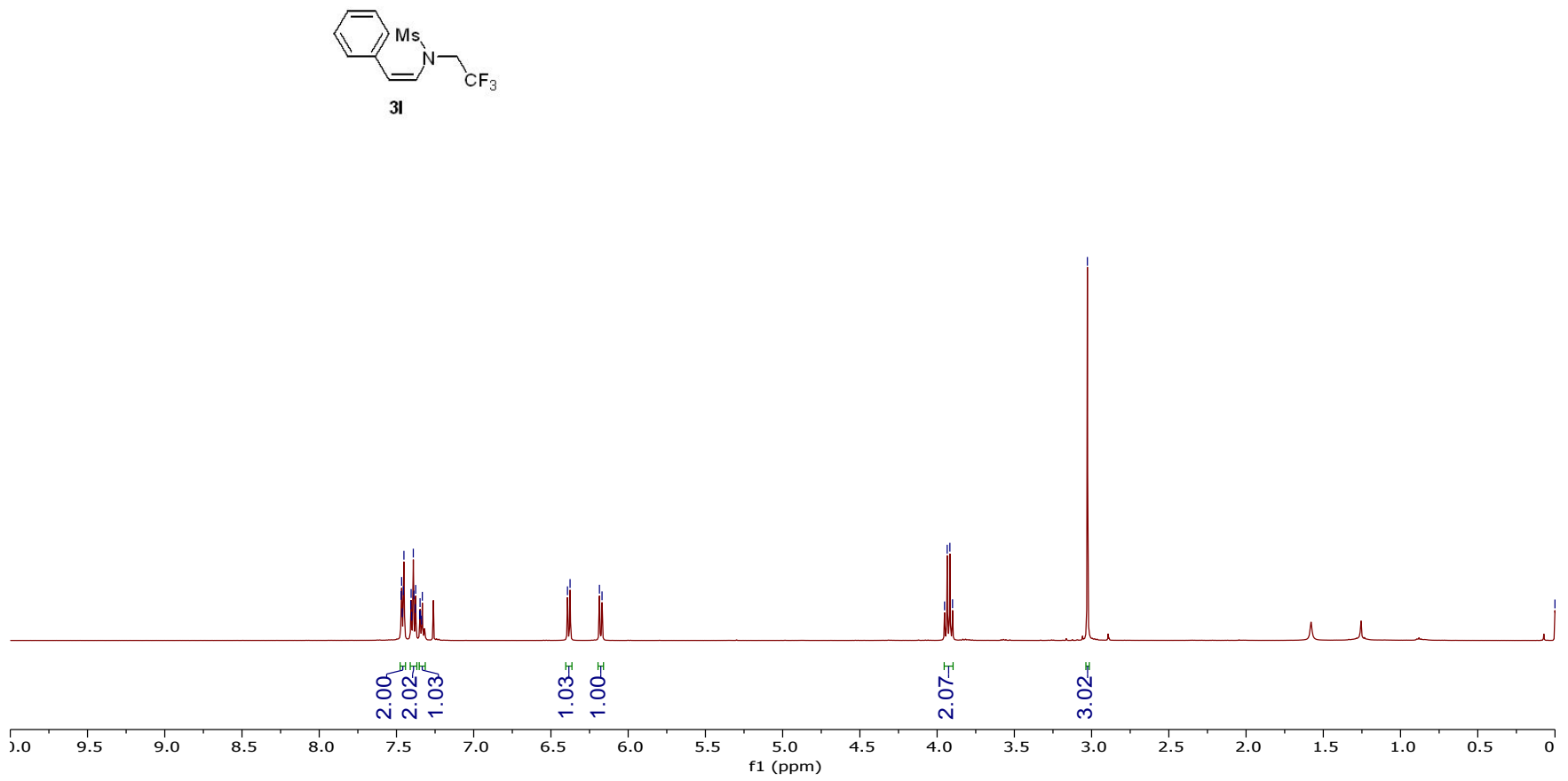

${ }^{13} \mathrm{C}\{1 \mathrm{H}\}$ NMR (126 $\left.\mathrm{MHz}, \mathrm{CDCl}_{3}\right)$ of $3 \mathrm{I}$

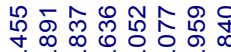

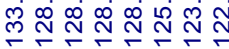

芯全亮

ヘิง

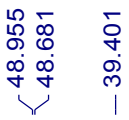
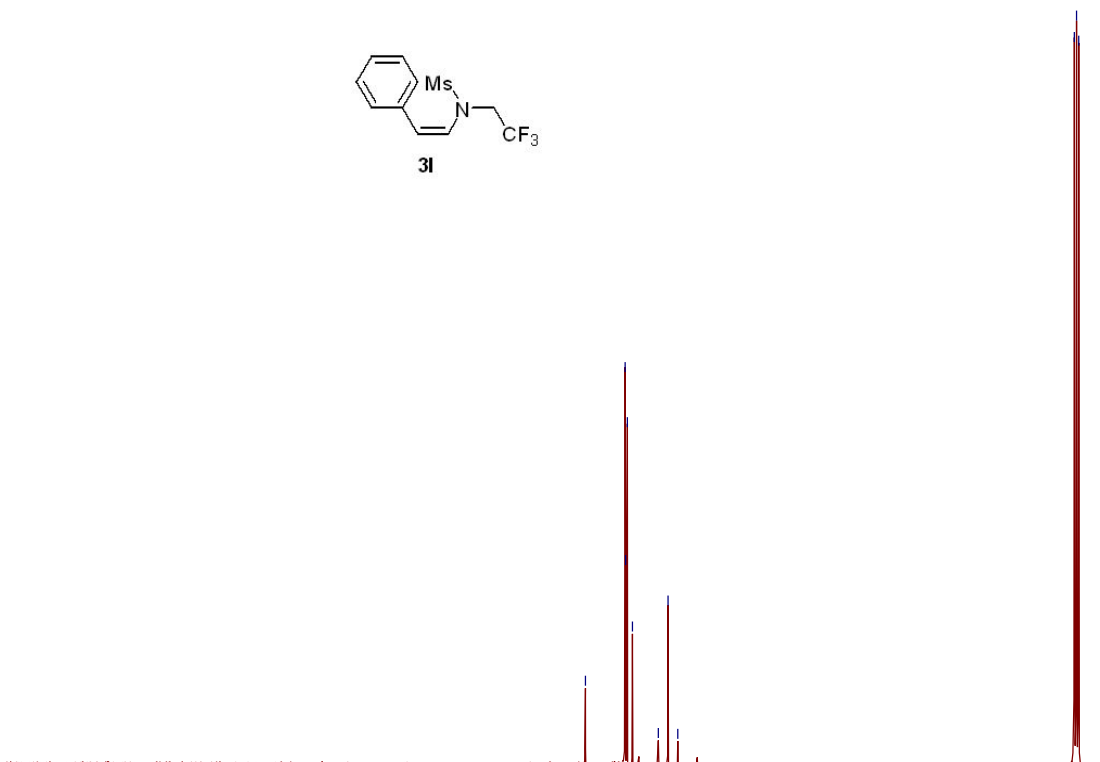

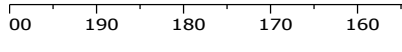

$150 \quad 140$

130

120

$110 \begin{gathered}100 \\ \mathrm{f} 1(\mathrm{ppm})\end{gathered}$

$\begin{array}{llll}10 & 1 & 10 & 1\end{array}$

50

40 $30 \div 20 \quad 10 \div$

HR-MS spectrum of 31 


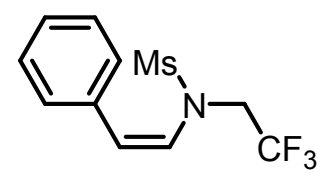

31

$[\mathrm{M}+\mathrm{H}]^{+}$Cal: 280.0614 , Found: 280.0613

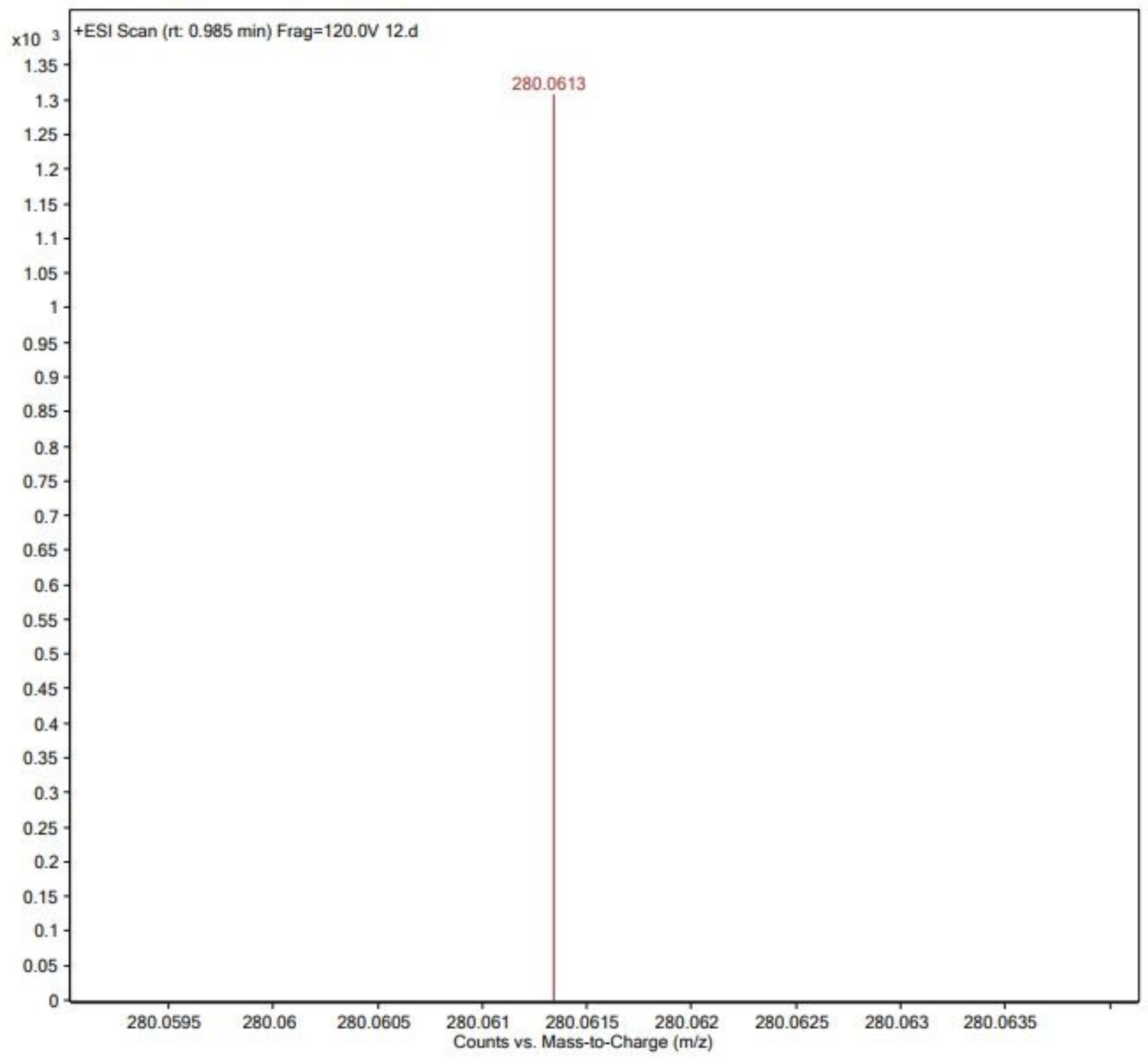




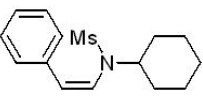

$3 m$
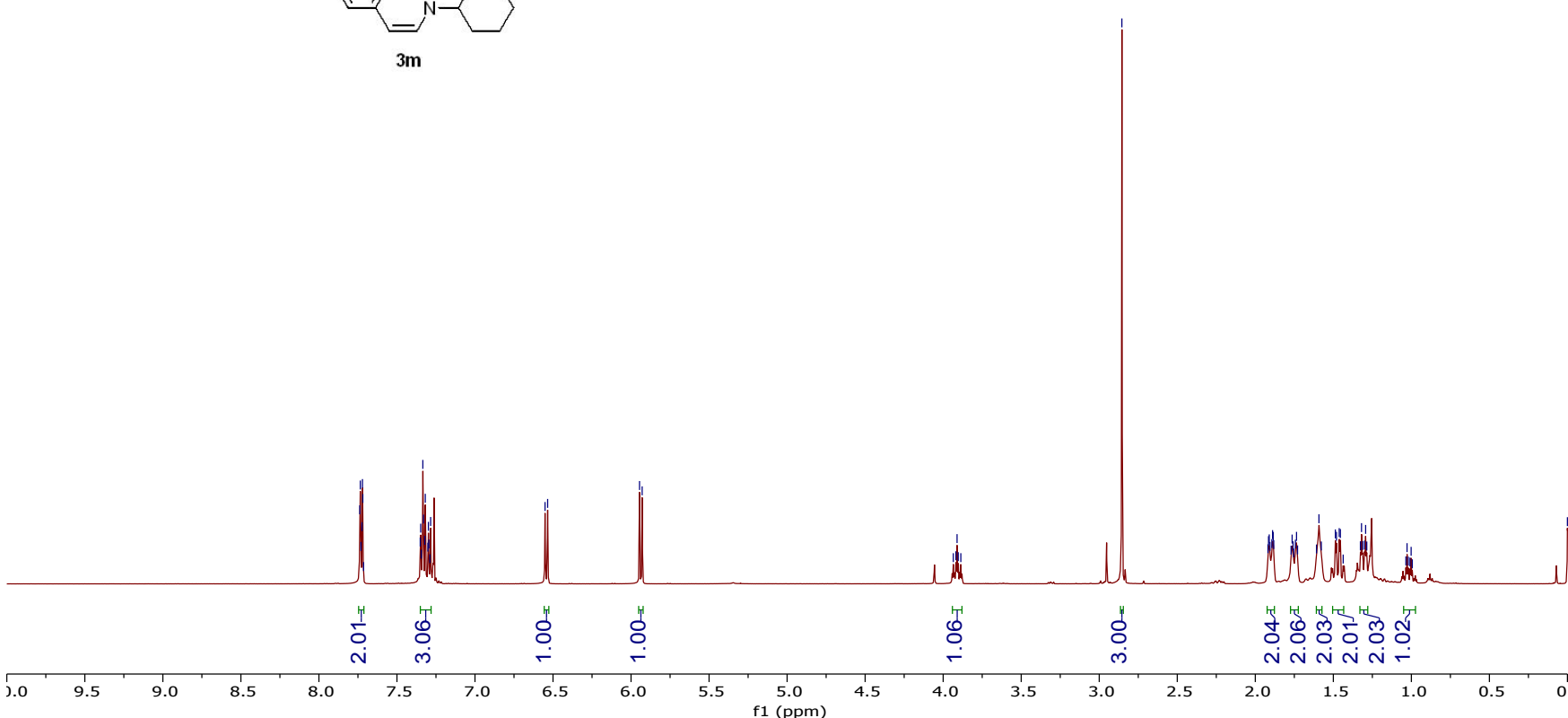

${ }^{13} \mathrm{C}\{1 \mathrm{H}\}$ NMR (126 MHz, $\left.\mathrm{CDCl}_{3}\right)$ of $3 \mathrm{~m}$

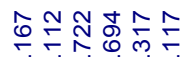

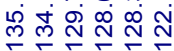

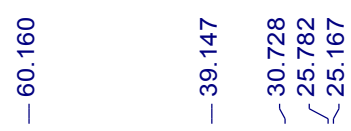

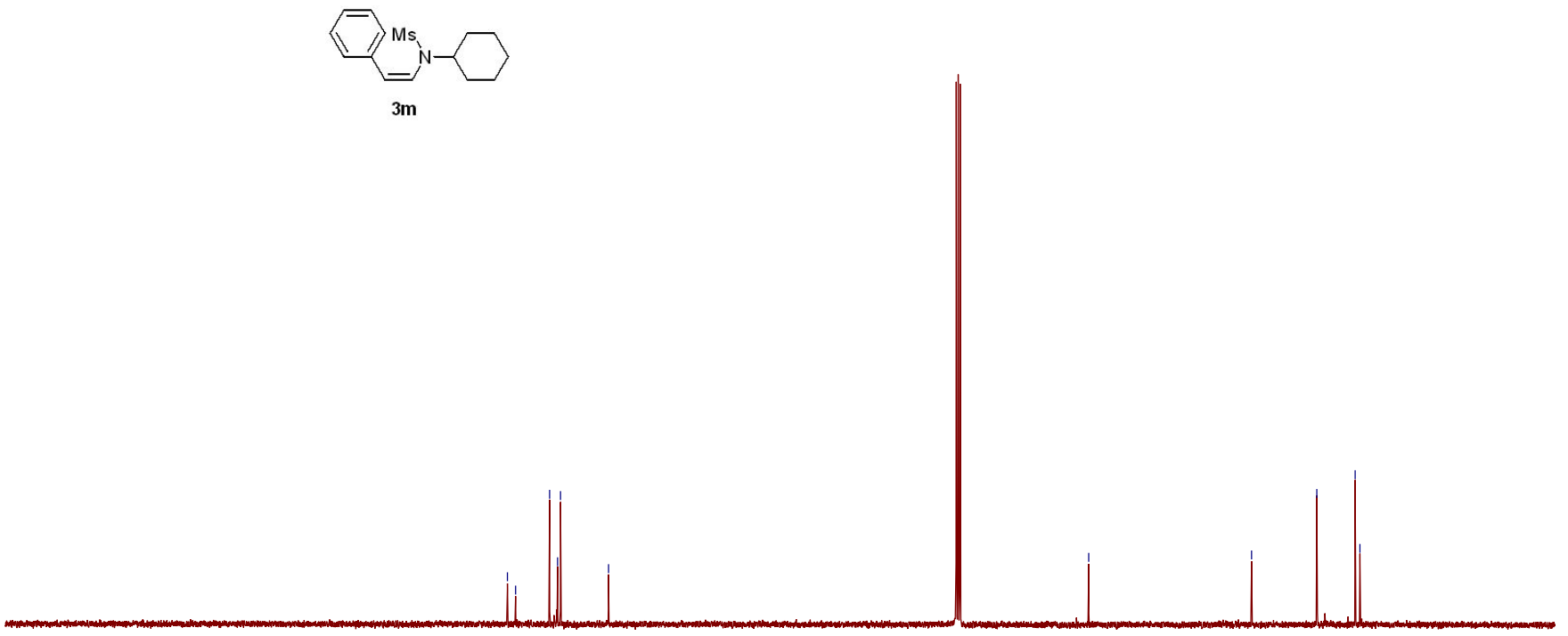

$3 \mathrm{~m}$

1)

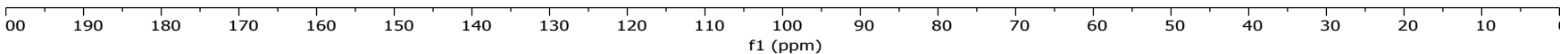




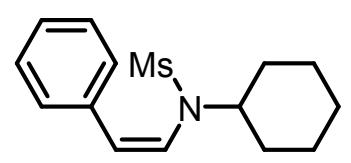

$3 \mathrm{~m}$

$[\mathrm{M}+\mathrm{H}]^{+}$Cal: 280.1366 , Found: 280.1364

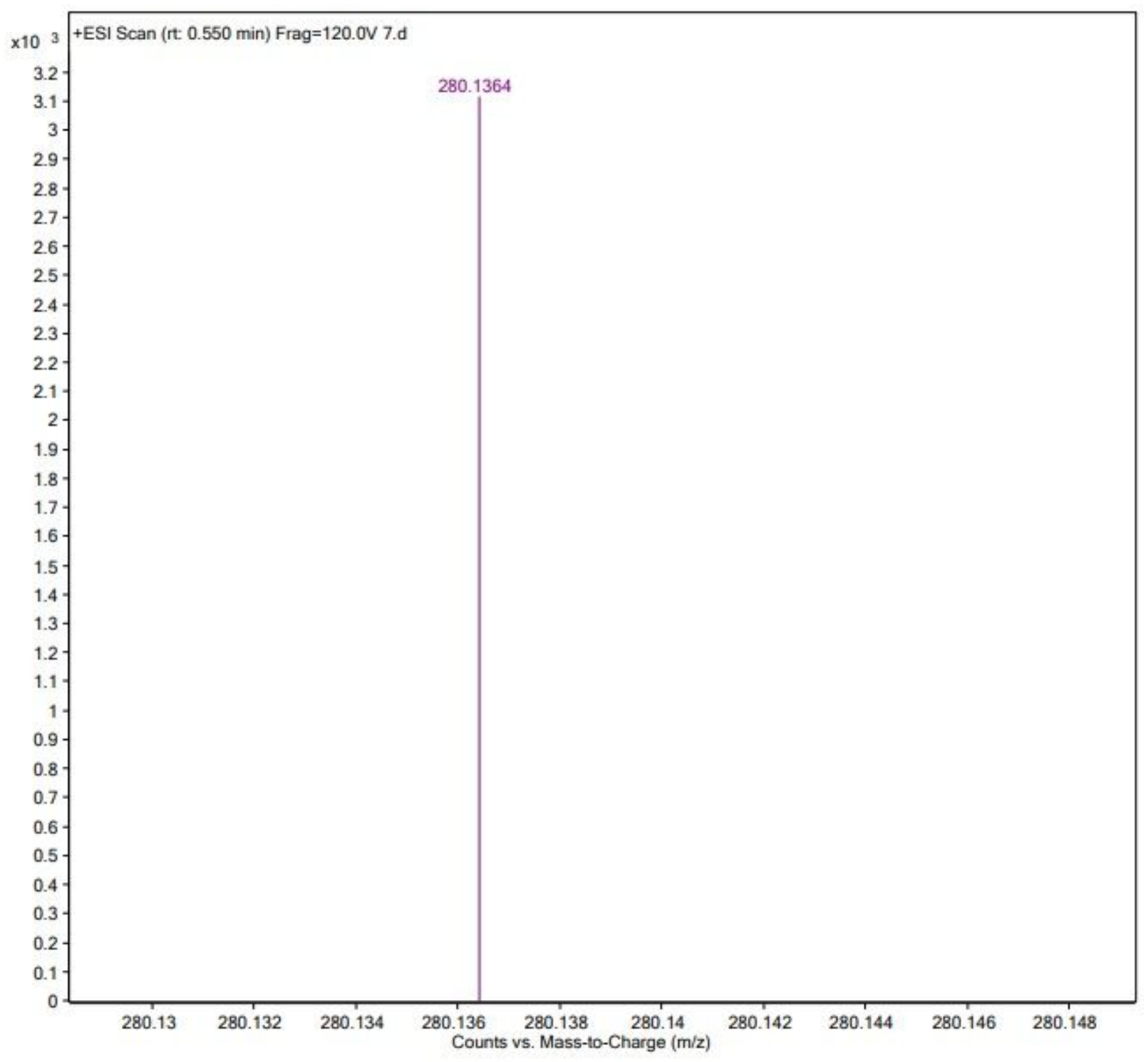




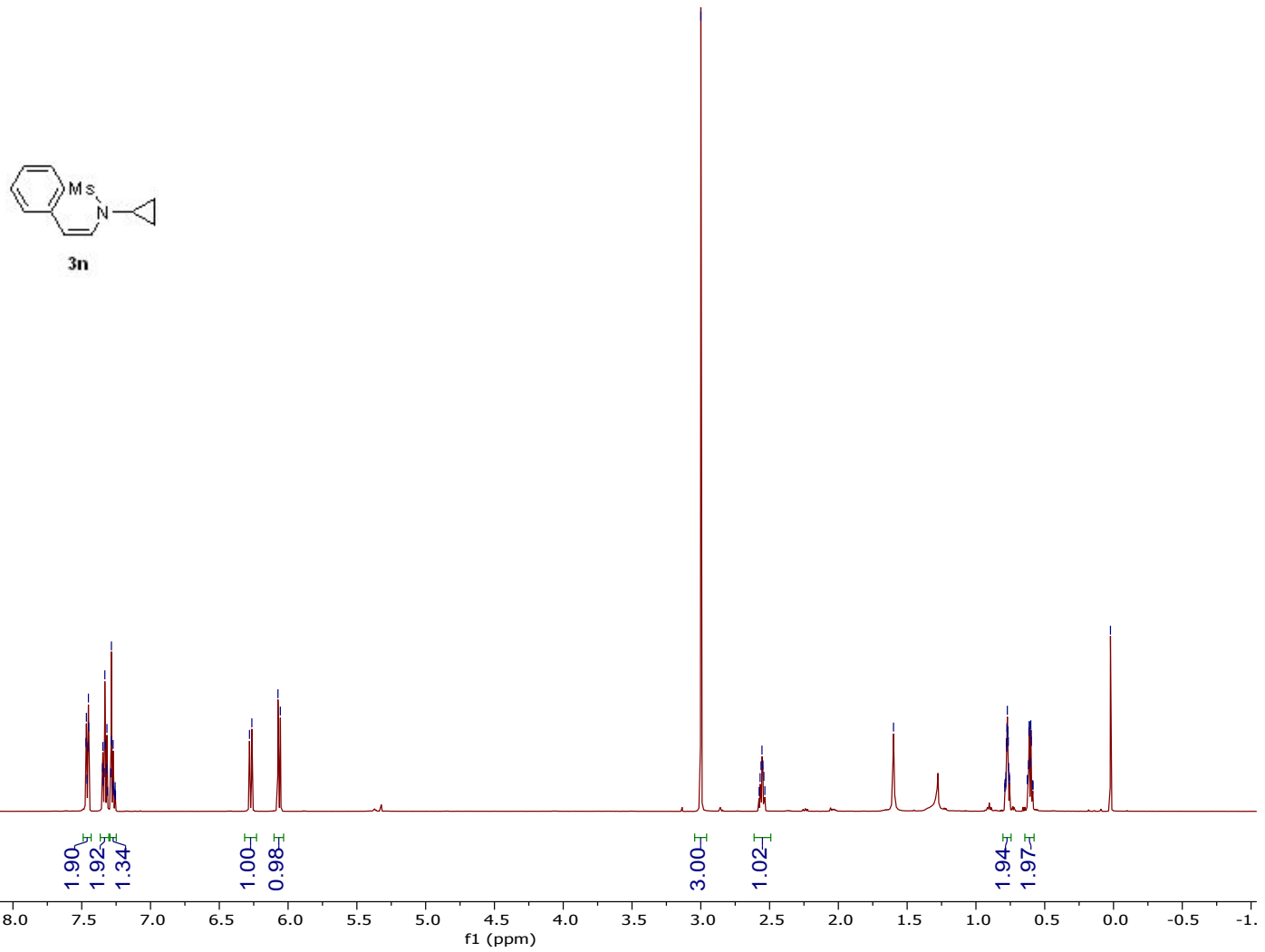

${ }^{13} \mathrm{C}\{1 \mathrm{H}\}$ NMR (126 $\left.\mathrm{MHz}, \mathrm{CDCl}_{3}\right)$ of 3n
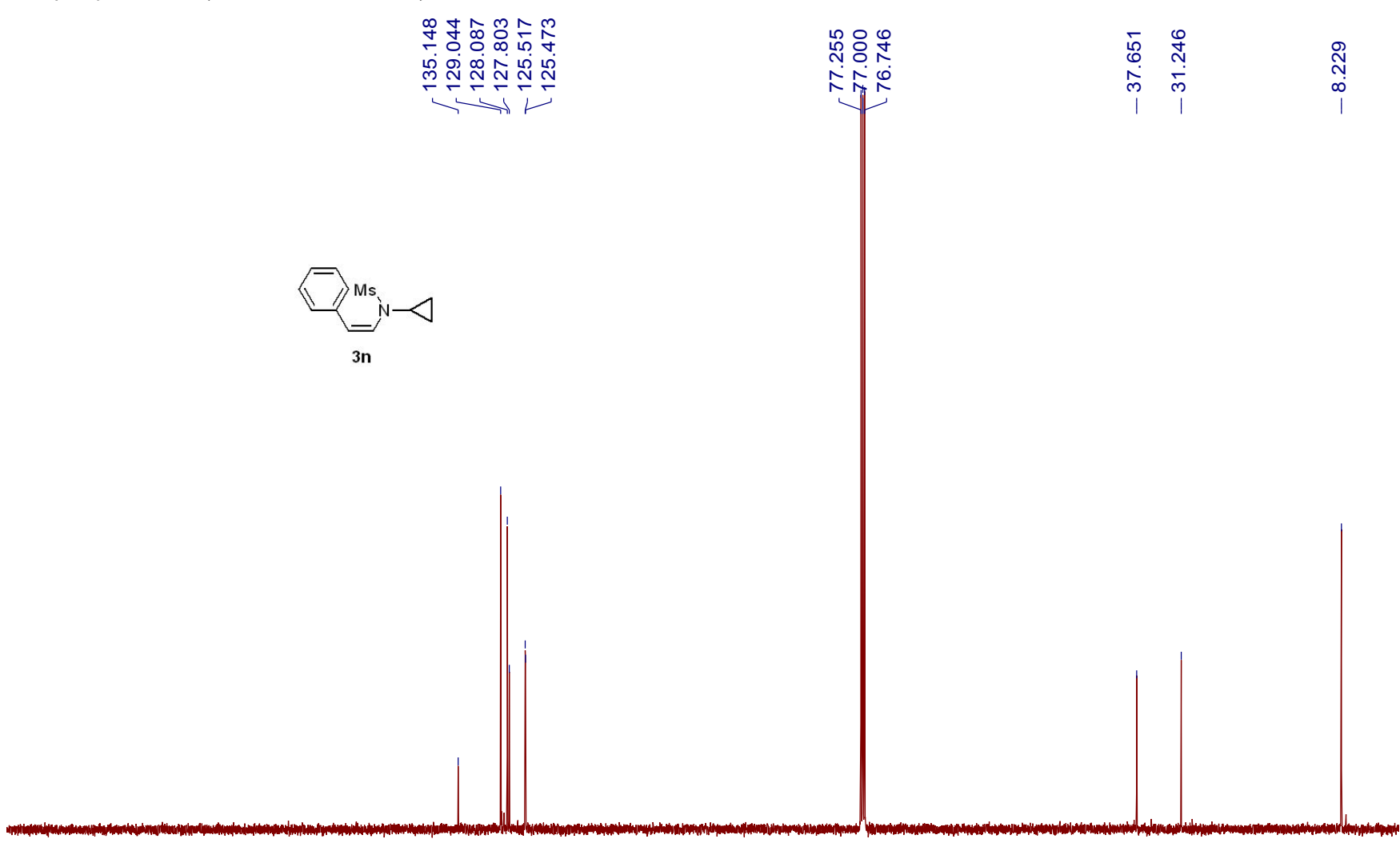

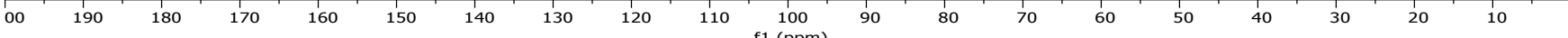

HR-MS spectrum of 3n 


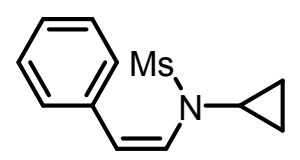

$3 n$

$[\mathrm{M}+\mathrm{H}]^{+}$Cal: 238.0896, Found: 238.0895

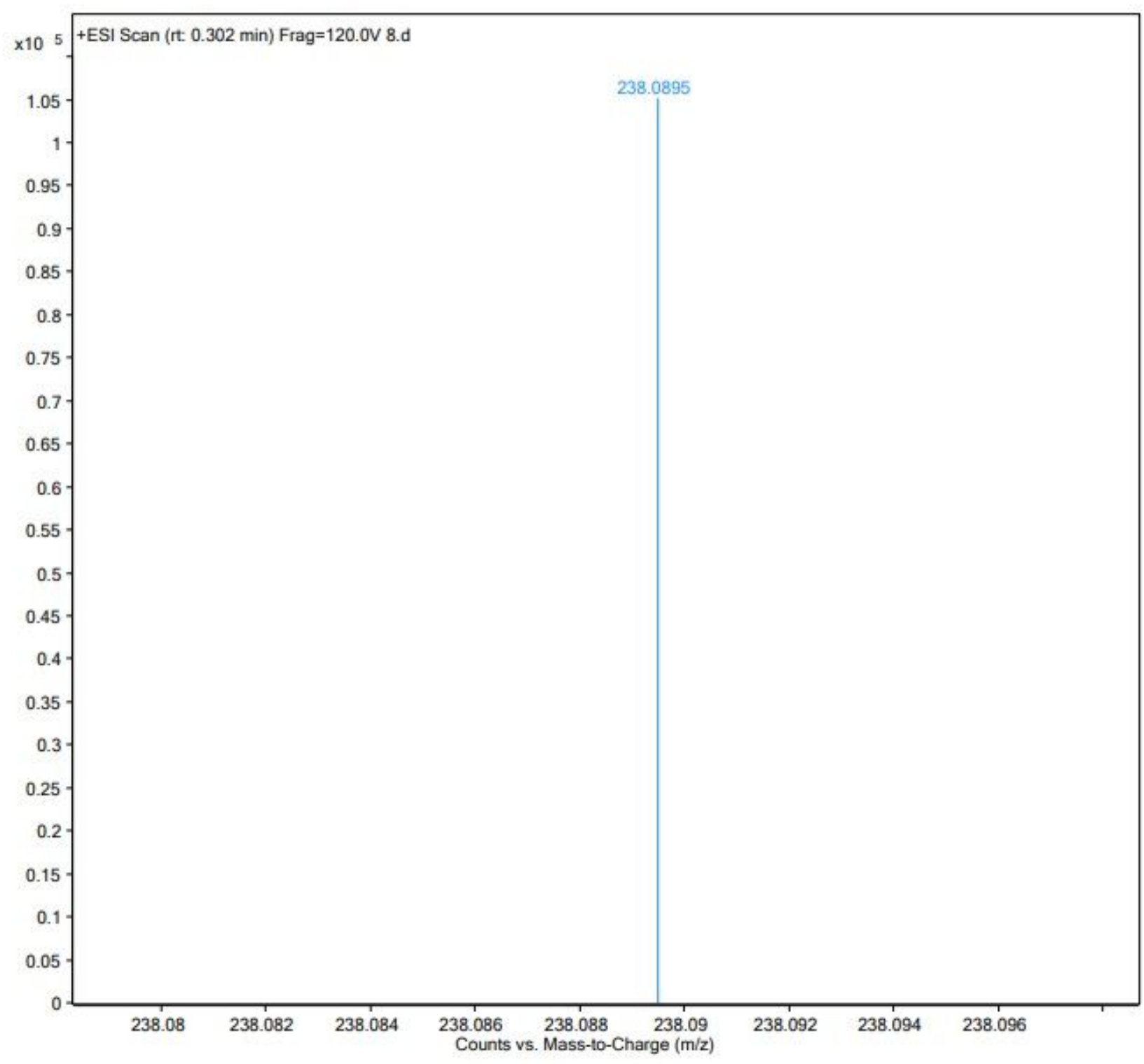




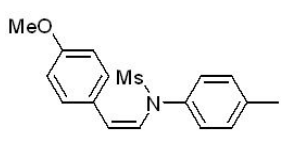

30

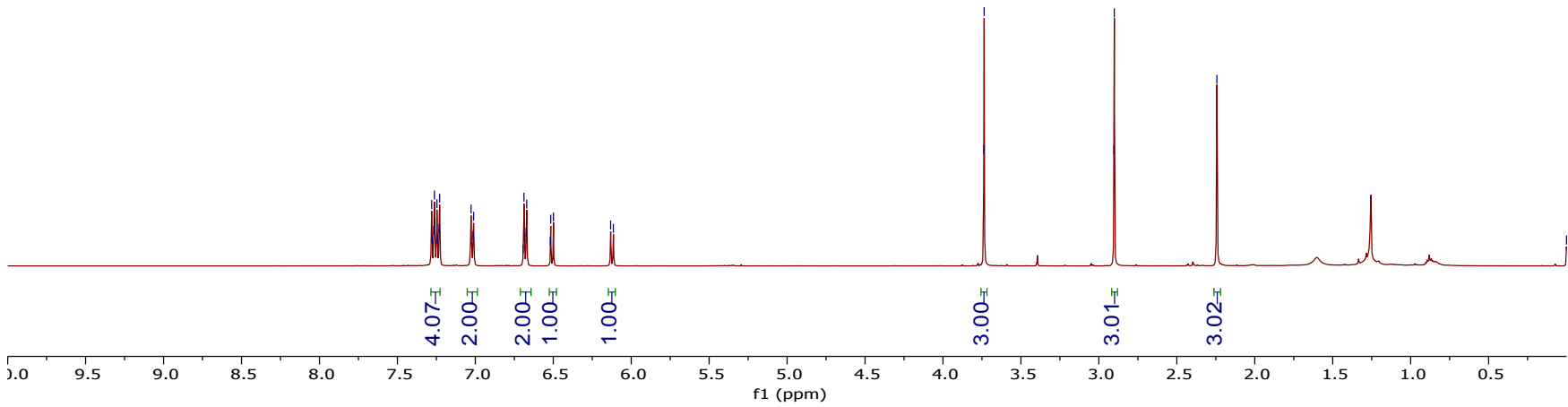

${ }^{13} \mathrm{C}\{1 \mathrm{H}\}$ NMR (126 $\left.\mathrm{MHz}, \mathrm{CDCl}_{3}\right)$ of 30

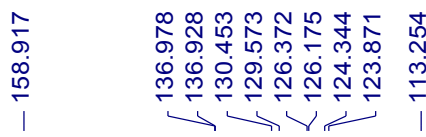

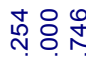

トरำ

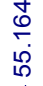

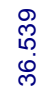

0
$N$
$\infty$
ஸे
1

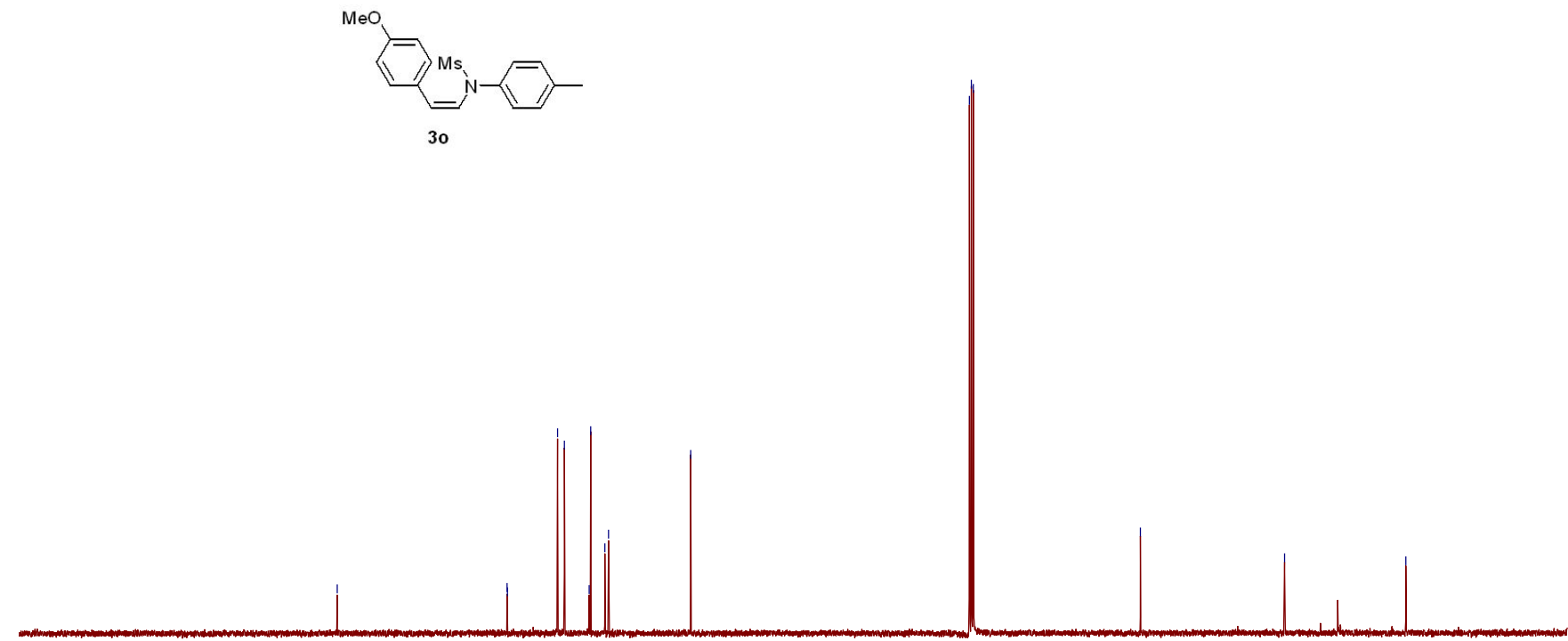

30

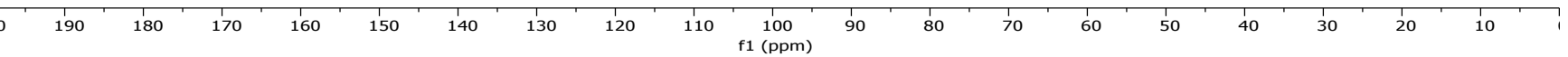




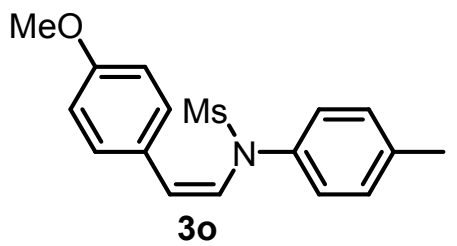

$[\mathrm{M}+\mathrm{H}]^{+}$Cal: 318.1158, Found: 318.1159

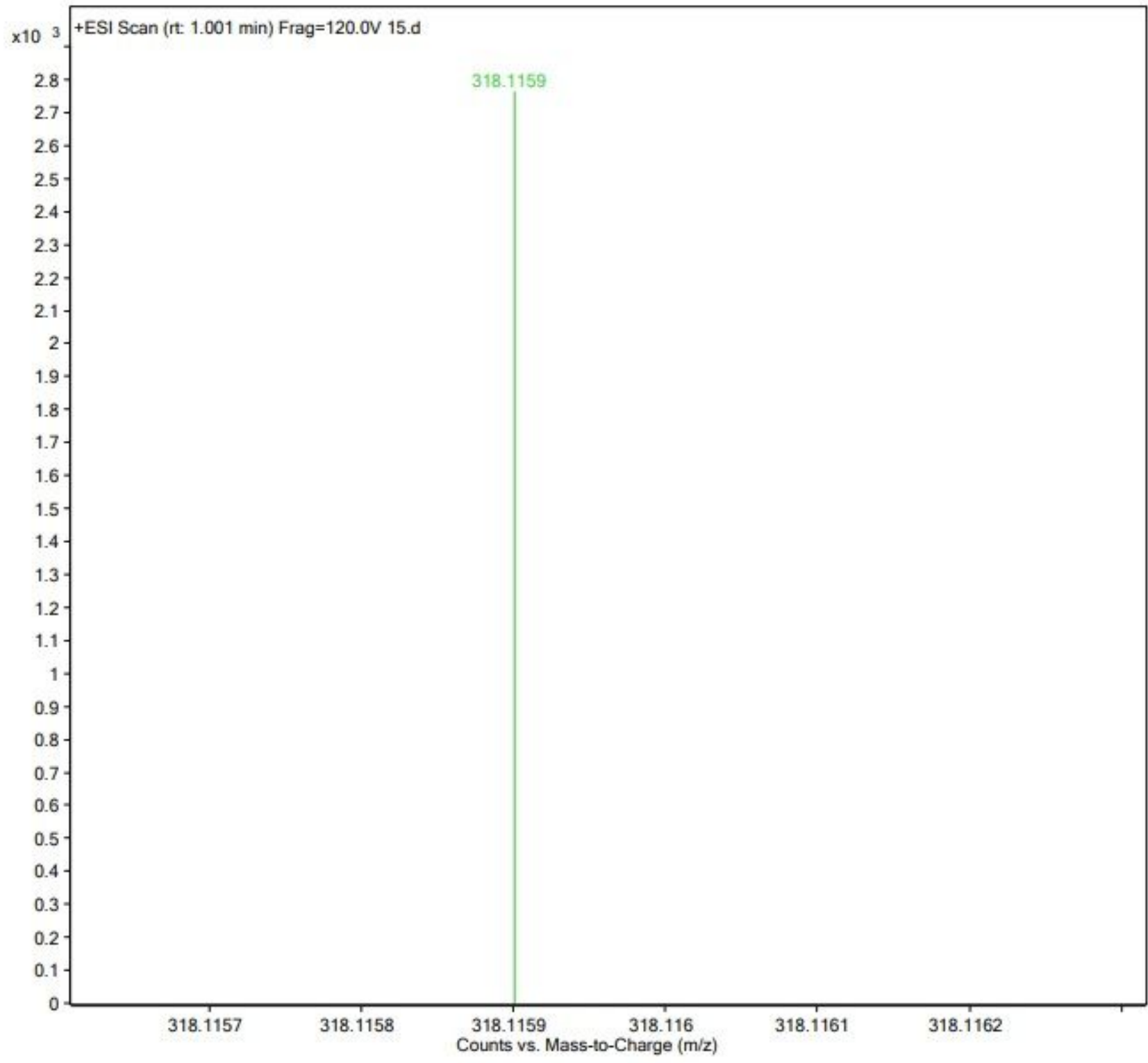

${ }^{1} \mathrm{H}$ NMR (500 MHz, $\mathrm{CDCl}_{3}$ ) of 3p 

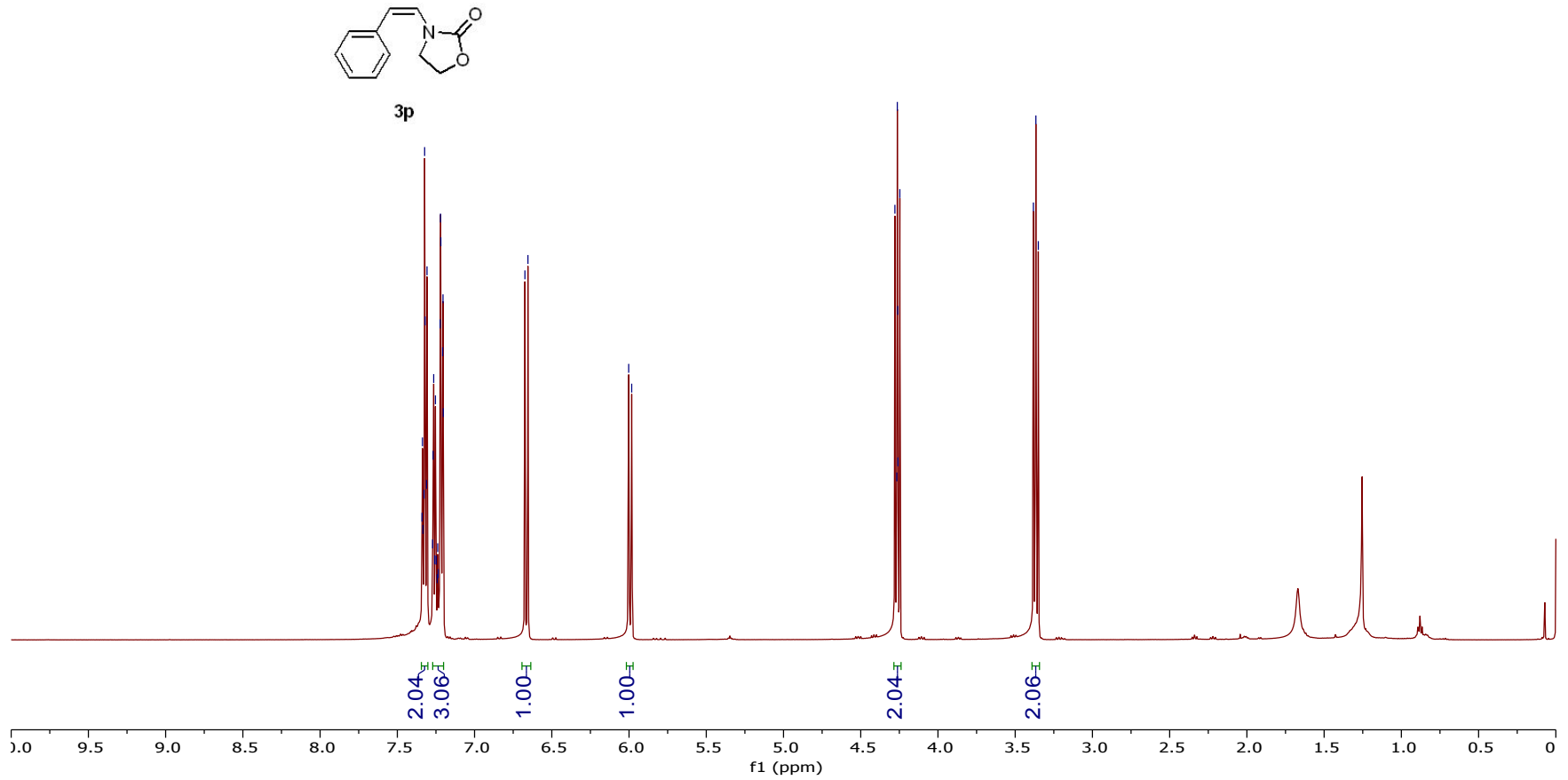

${ }^{13} \mathrm{C}\{1 \mathrm{H}\}$ NMR (126 $\left.\mathrm{MHz}, \mathrm{CDCl}_{3}\right)$ of 3p

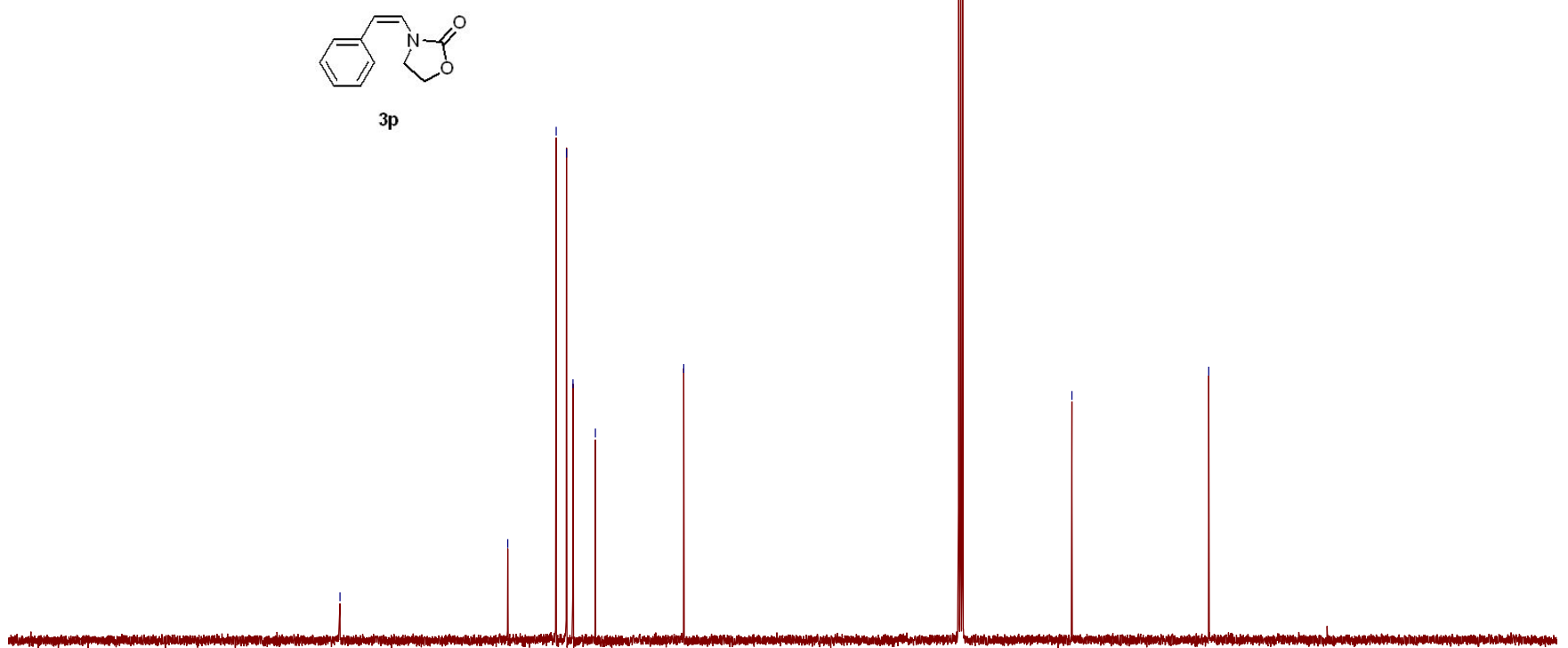

$3 p$ 


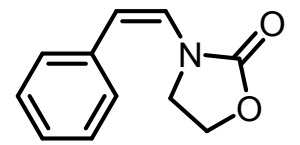

$3 p$

$[\mathrm{M}+\mathrm{H}]^{+}$Cal: 190.0863 , Found: 190.0862

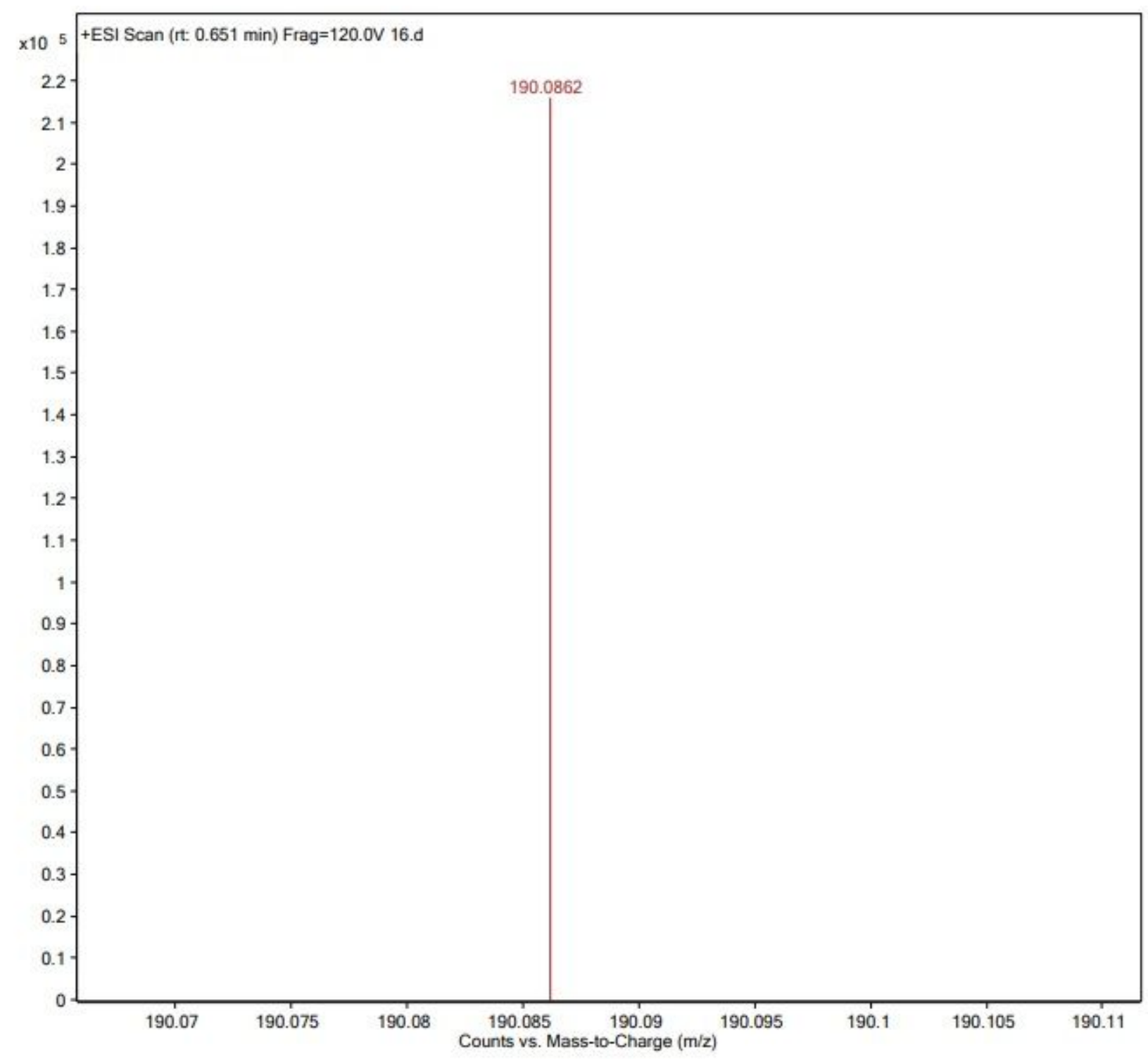




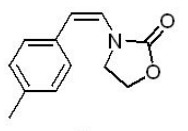

$3 q$

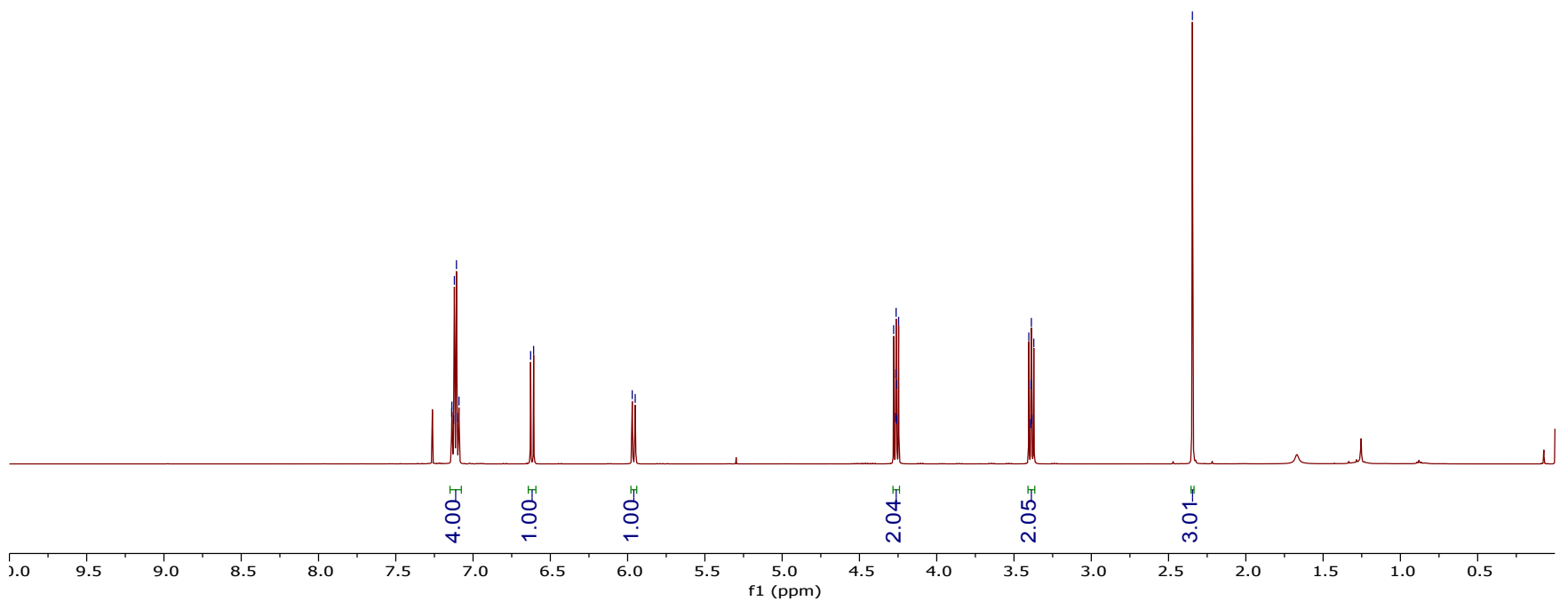

${ }^{13} \mathrm{C}\{1 \mathrm{H}\}$ NMR (126 $\left.\mathrm{MHz}, \mathrm{CDCl}_{3}\right)$ of $3 \mathrm{q}$

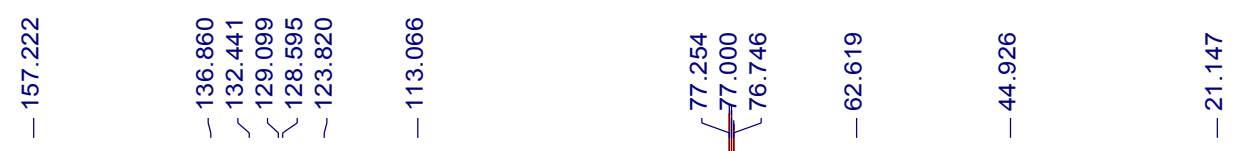

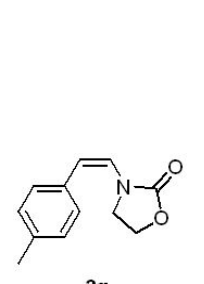

$3 q$

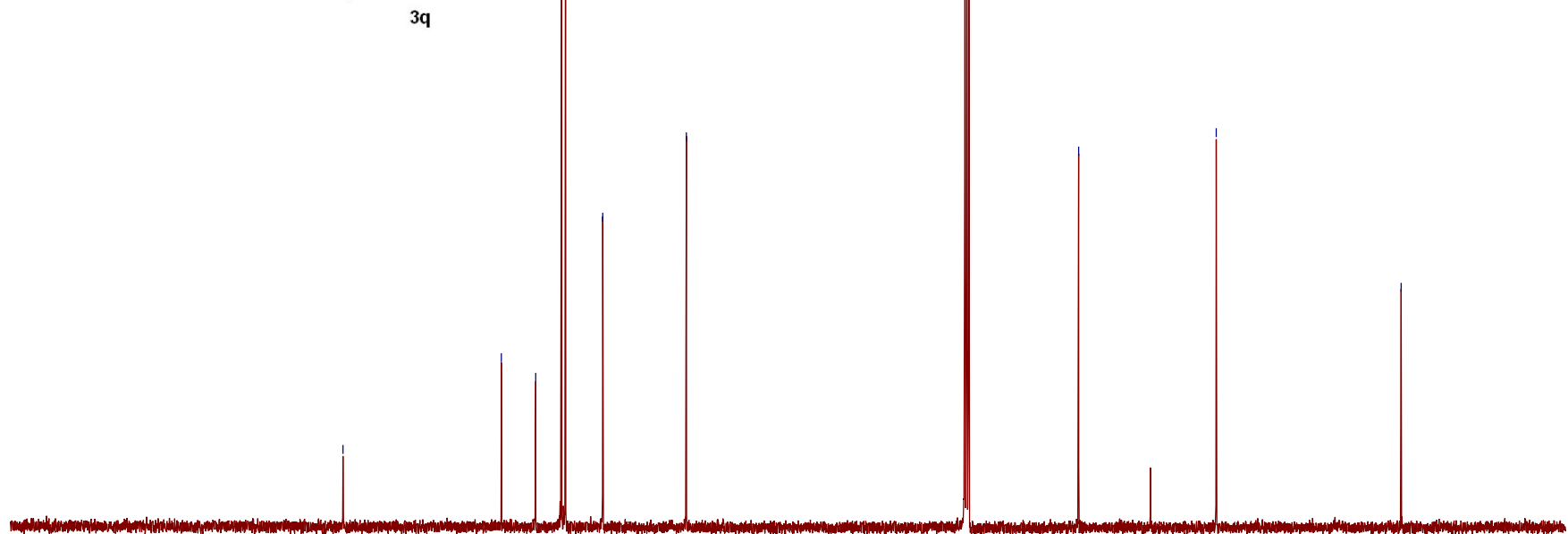

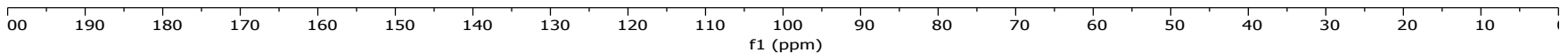

HR-MS spectrum of 3q 


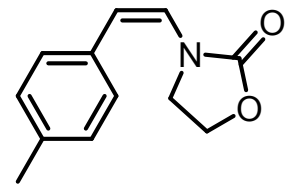

$3 q$

$[\mathrm{M}+\mathrm{H}]^{+}$Cal: 204.1019, Found: 204.1020

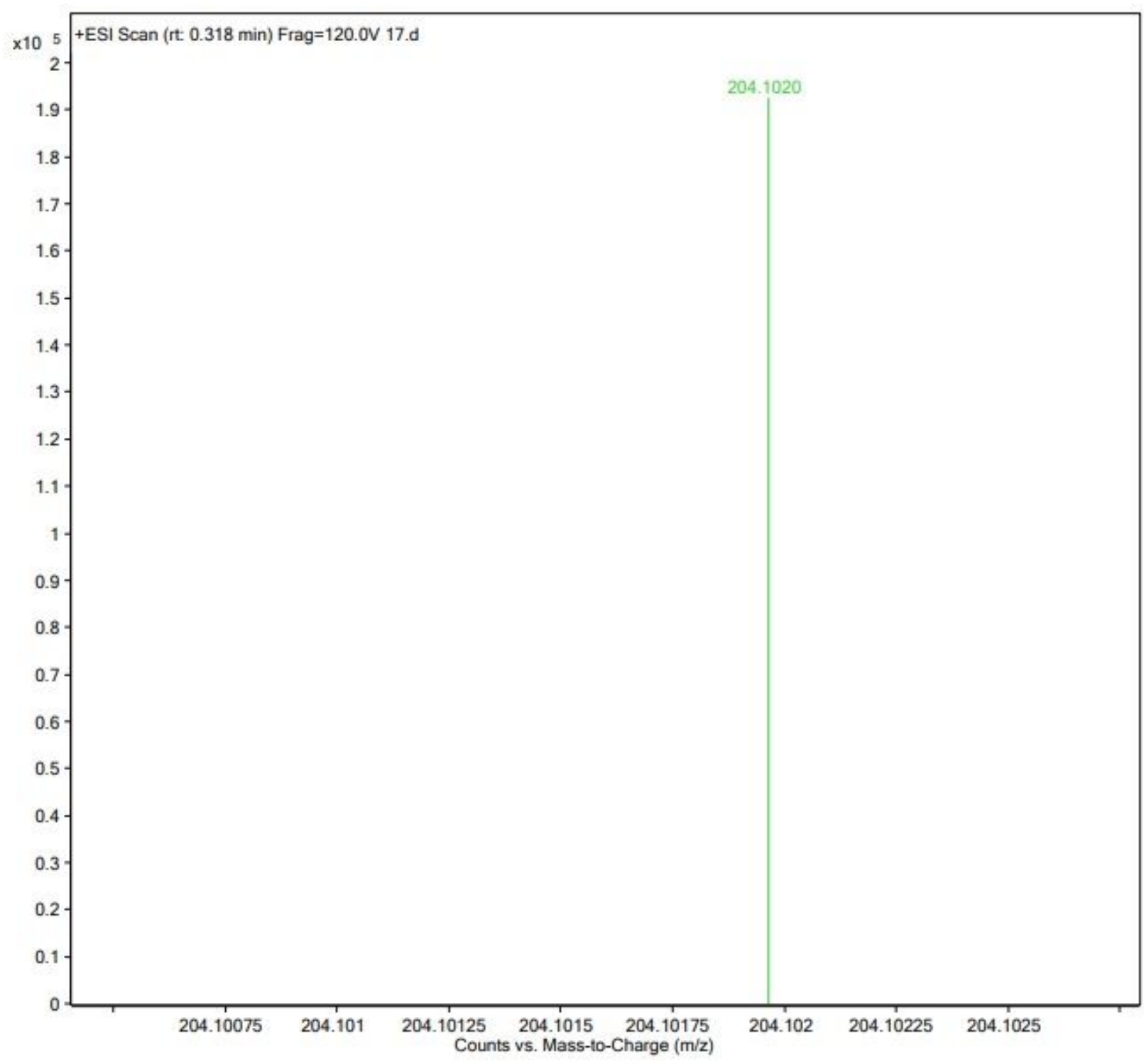

${ }^{1} \mathrm{H}$ NMR (500 MHz, $\left.\mathrm{CDCl}_{3}\right)$ of 3r 


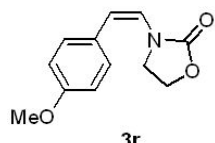

$3 r$

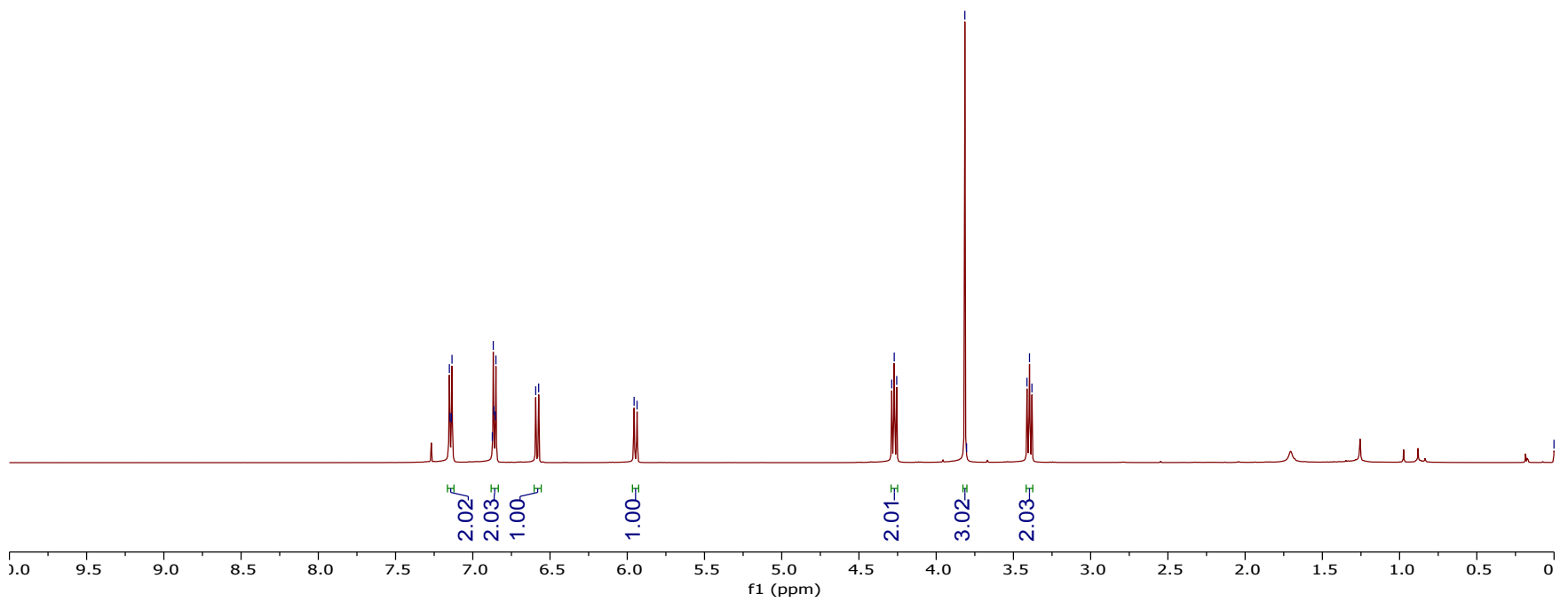

${ }^{13} \mathrm{C}\{1 \mathrm{H}\}$ NMR (126 MHz, $\left.\mathrm{CDCl}_{3}\right)$ of $3 \mathrm{r}$

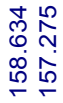

స్లి గ్రి

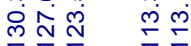

1

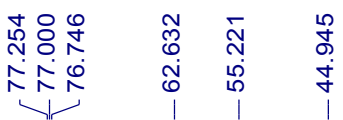

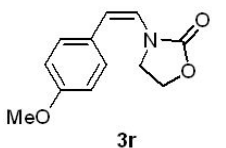

$3 r$

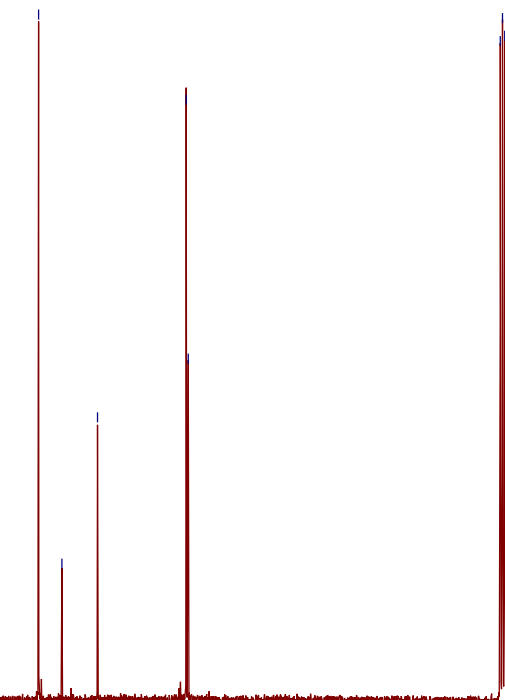

00

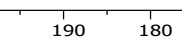

$180 \quad 170$

160

150

130

120

110

100

1
$90 \quad 80$

HR-MS spectrum of $3 r$ 


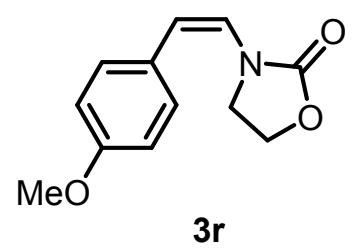

$[\mathrm{M}+\mathrm{H}]^{+}$Cal: 220.0968, Found: 220.0967

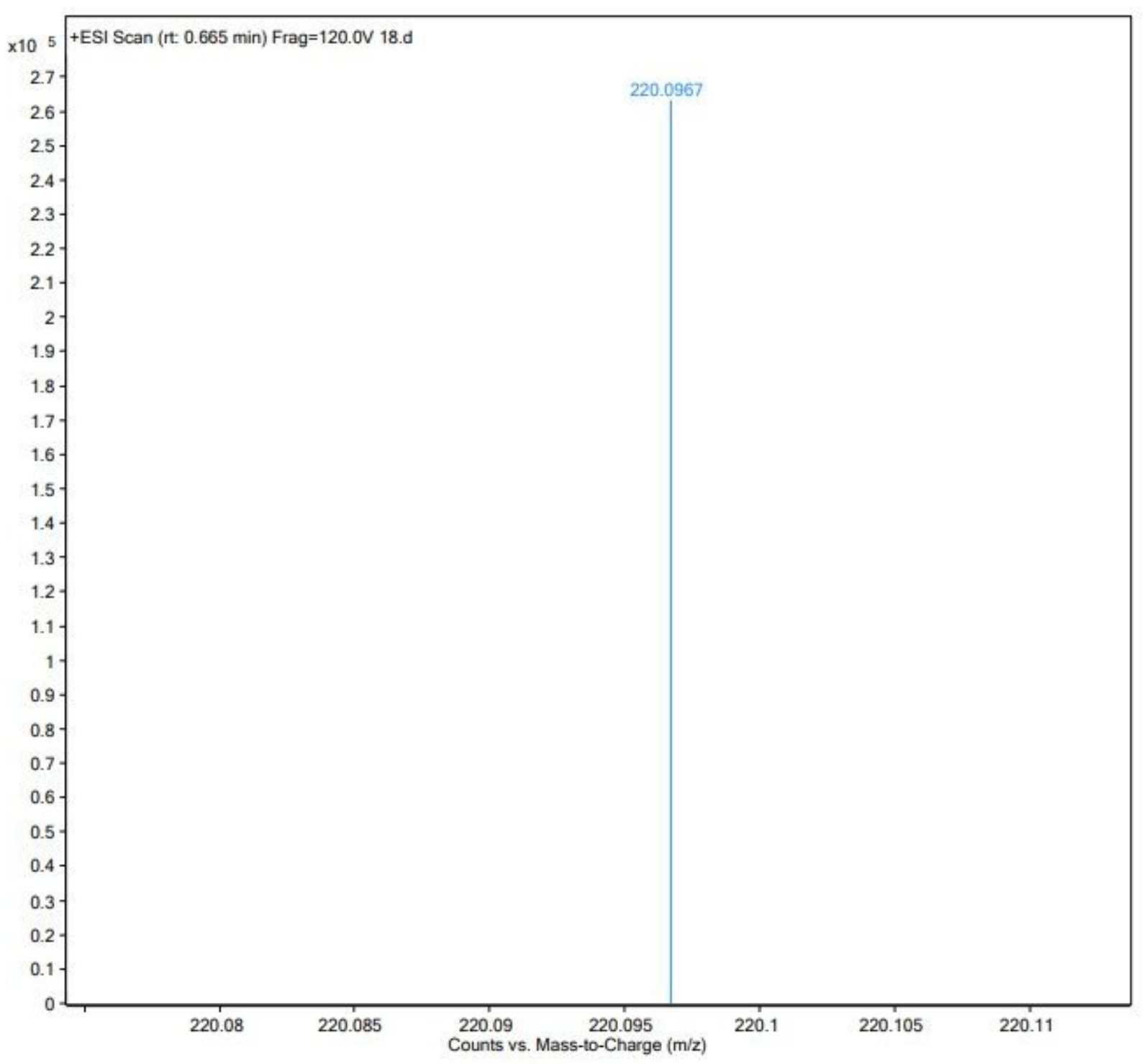



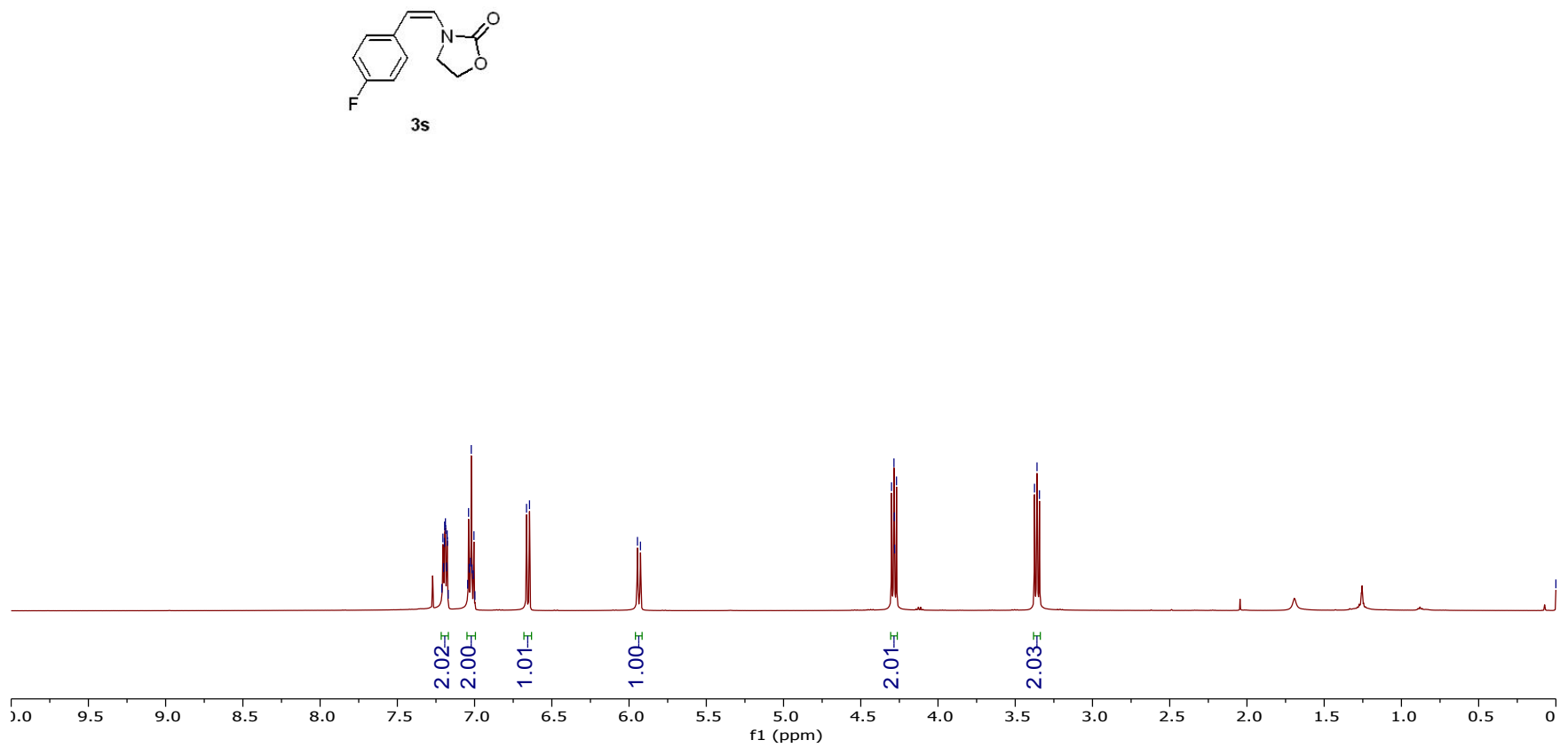

${ }^{13} \mathrm{C}\{1 \mathrm{H}\}$ NMR (126 $\left.\mathrm{MHz}, \mathrm{CDCl}_{3}\right)$ of $3 \mathrm{~s}$

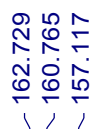

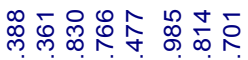

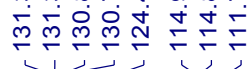

究等客

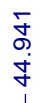

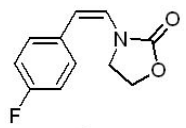

3s

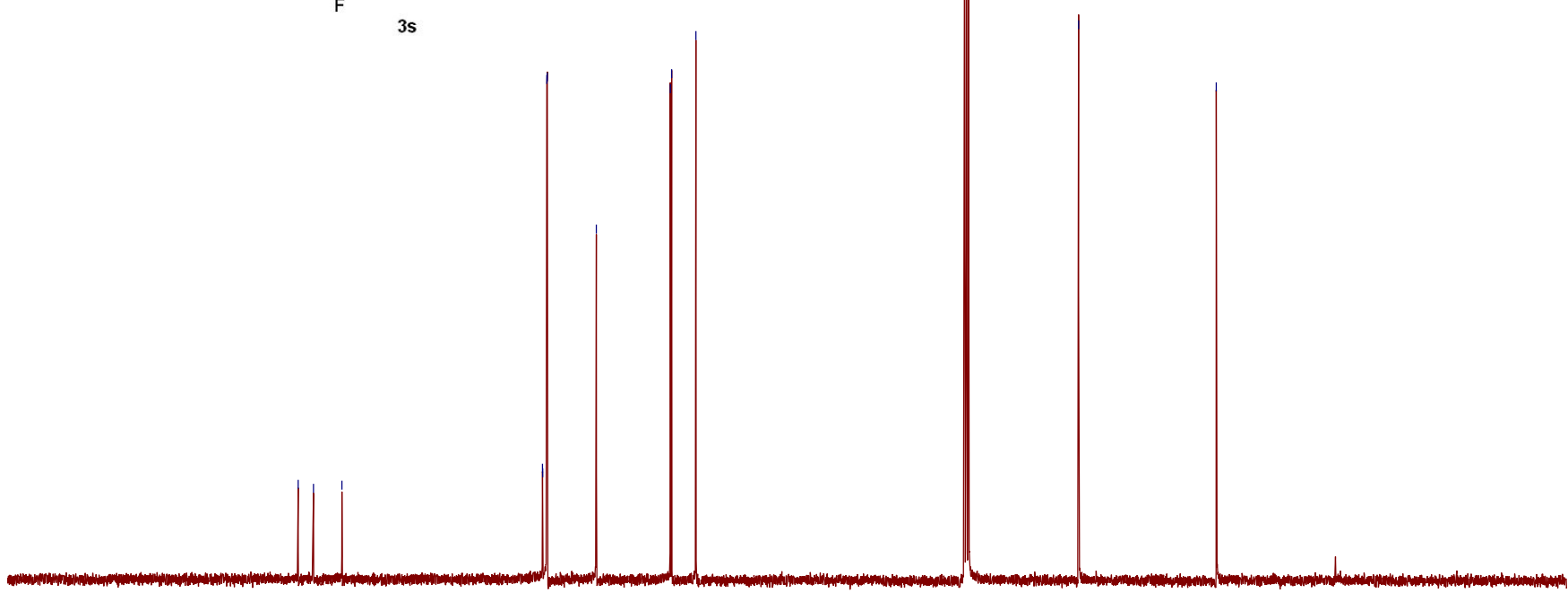

oo $190 \quad 180$

$100 \quad 90 \quad 80$

$\begin{array}{lllllll}70 & 60 & 50 & 40 & 30 & 20 & 10\end{array}$

HR-MS spectrum of $3 \mathrm{~s}$ 


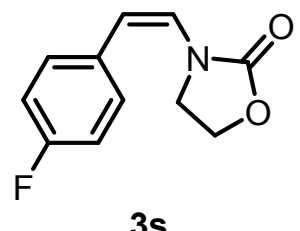

$3 s$

$[\mathrm{M}+\mathrm{H}]^{+}$Cal: 208.0768 , Found: 208.0770

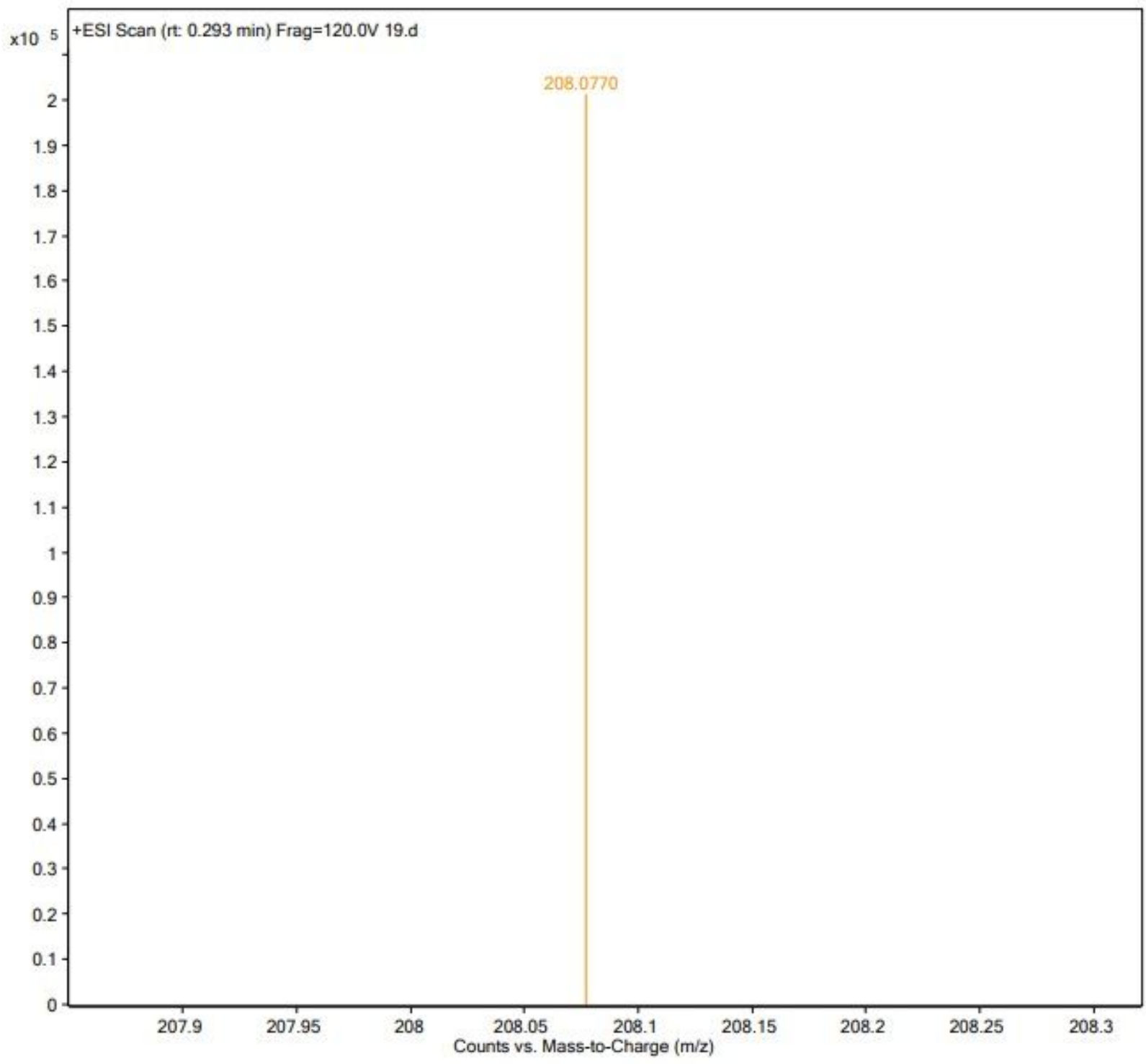



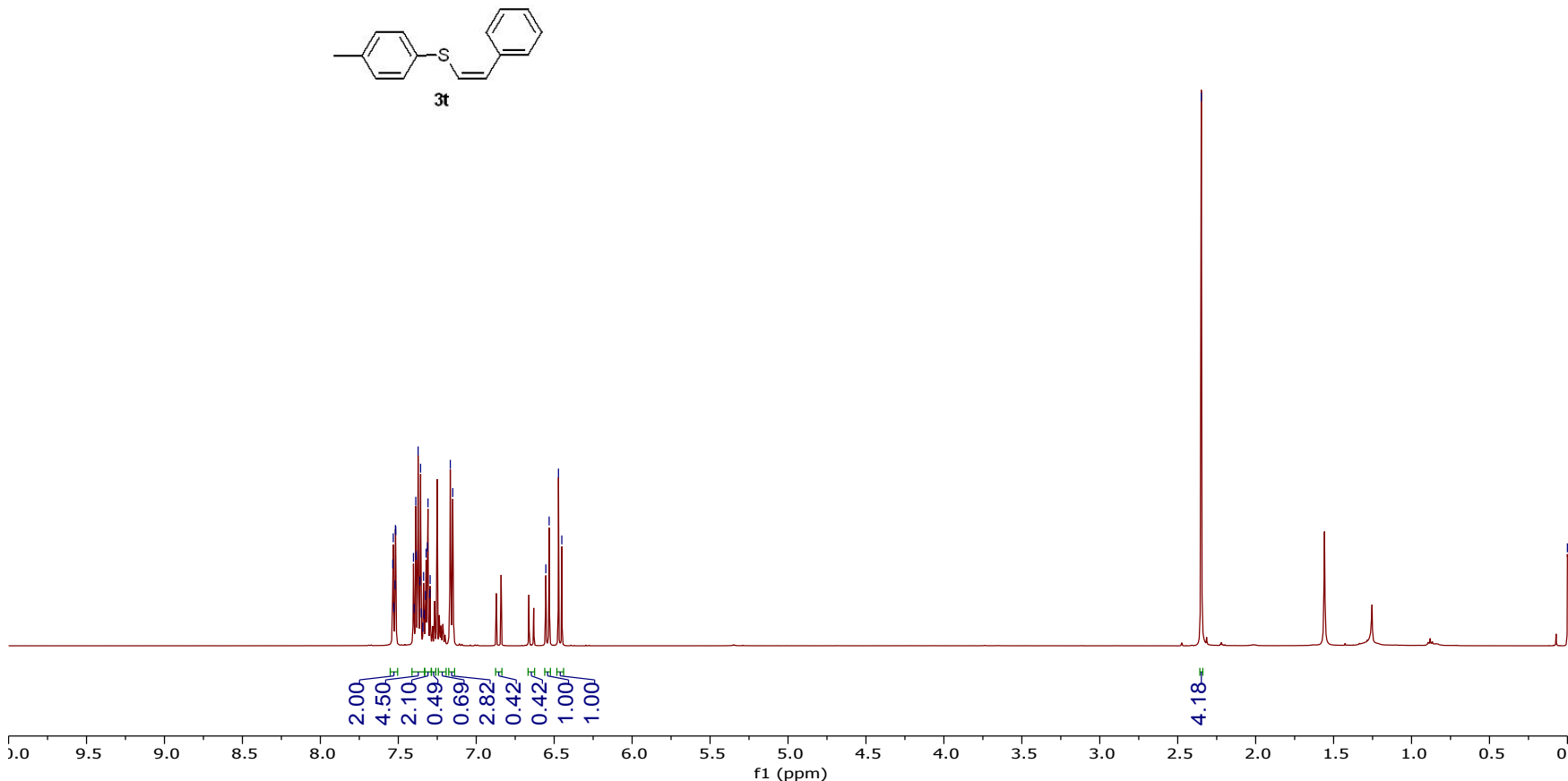

${ }^{13} \mathrm{C}\{1 \mathrm{H}\}$ NMR (126 MHz, $\left.\mathrm{CDCl}_{3}\right)$ of $3 \mathrm{t}$

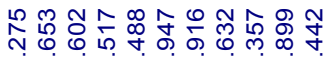

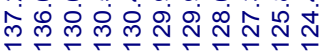

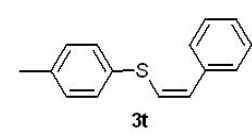

$3 t$

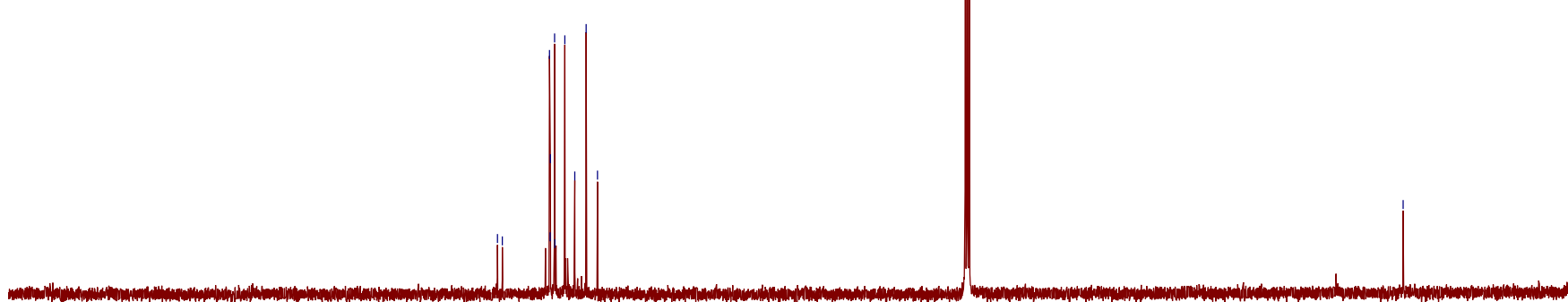




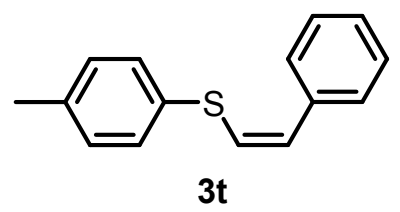

$[\mathrm{M}+\mathrm{H}]^{+}$Cal: 227.0889, Found: 227.0884

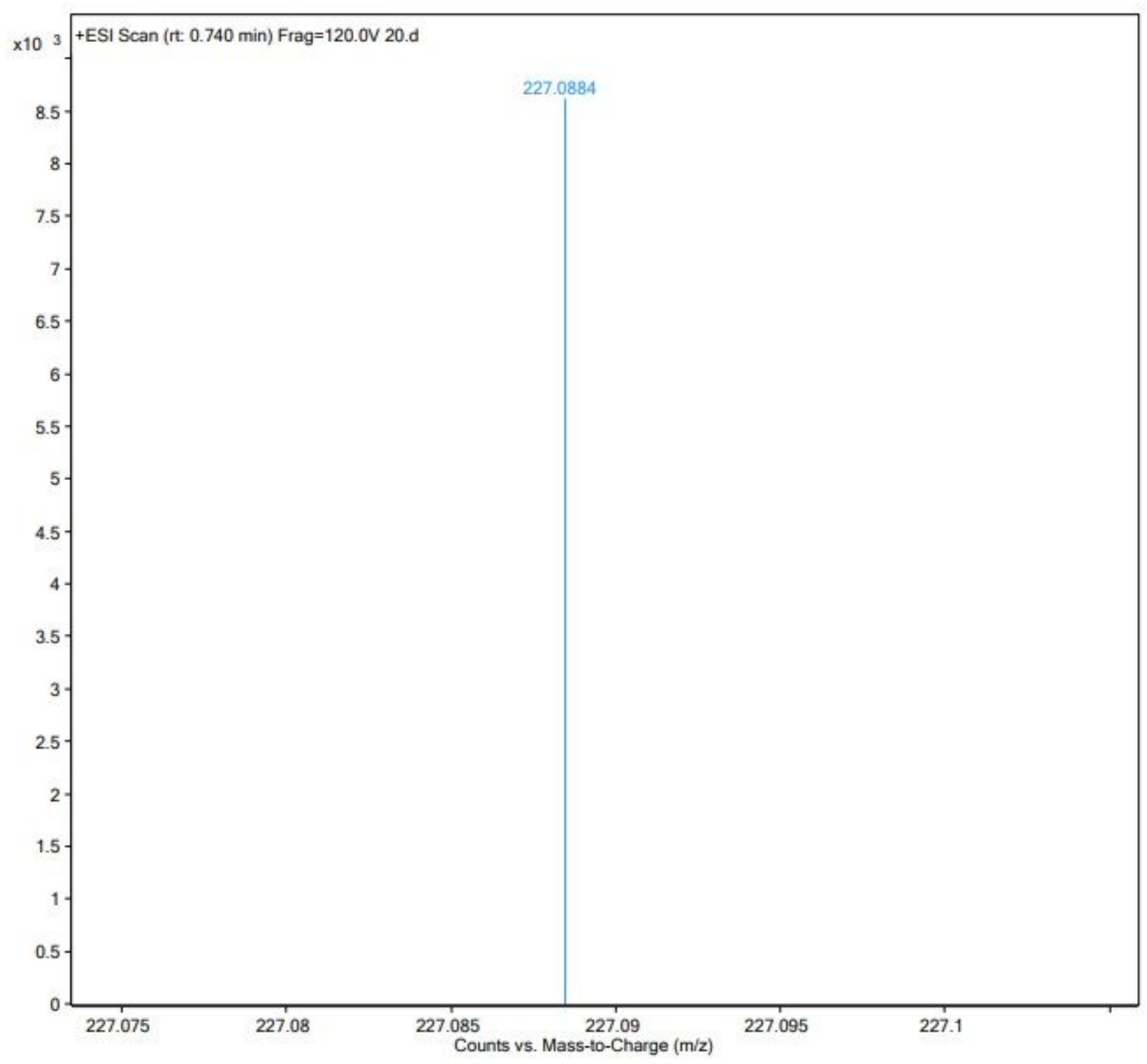




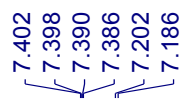

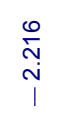
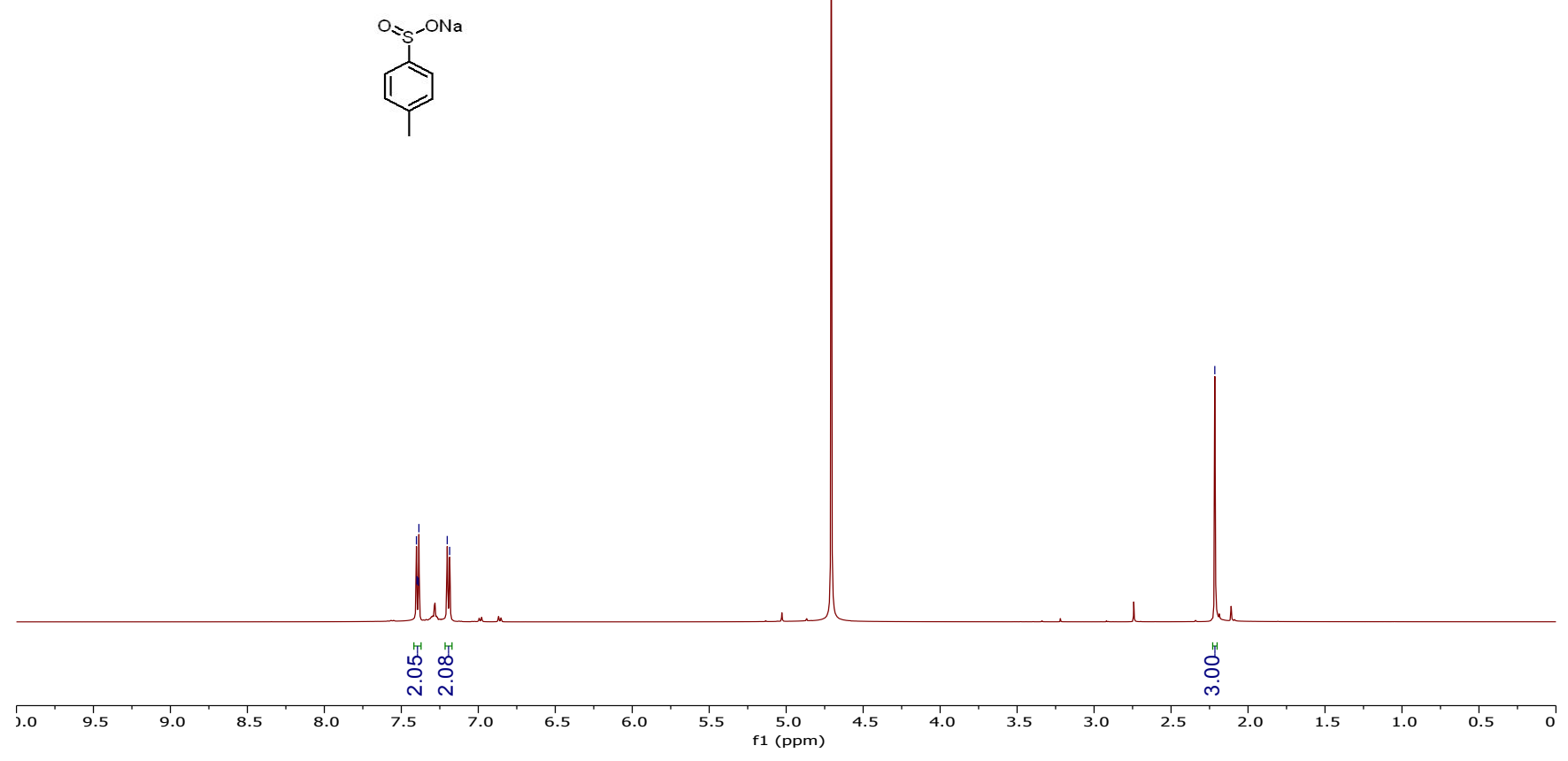

${ }^{13} \mathrm{C}\{1 \mathrm{H}\}$ NMR (126 MHz, $\left.\mathrm{D}_{2} \mathrm{O}\right)$ of sodium 4-methylbenzenesulfinate

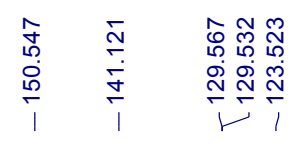


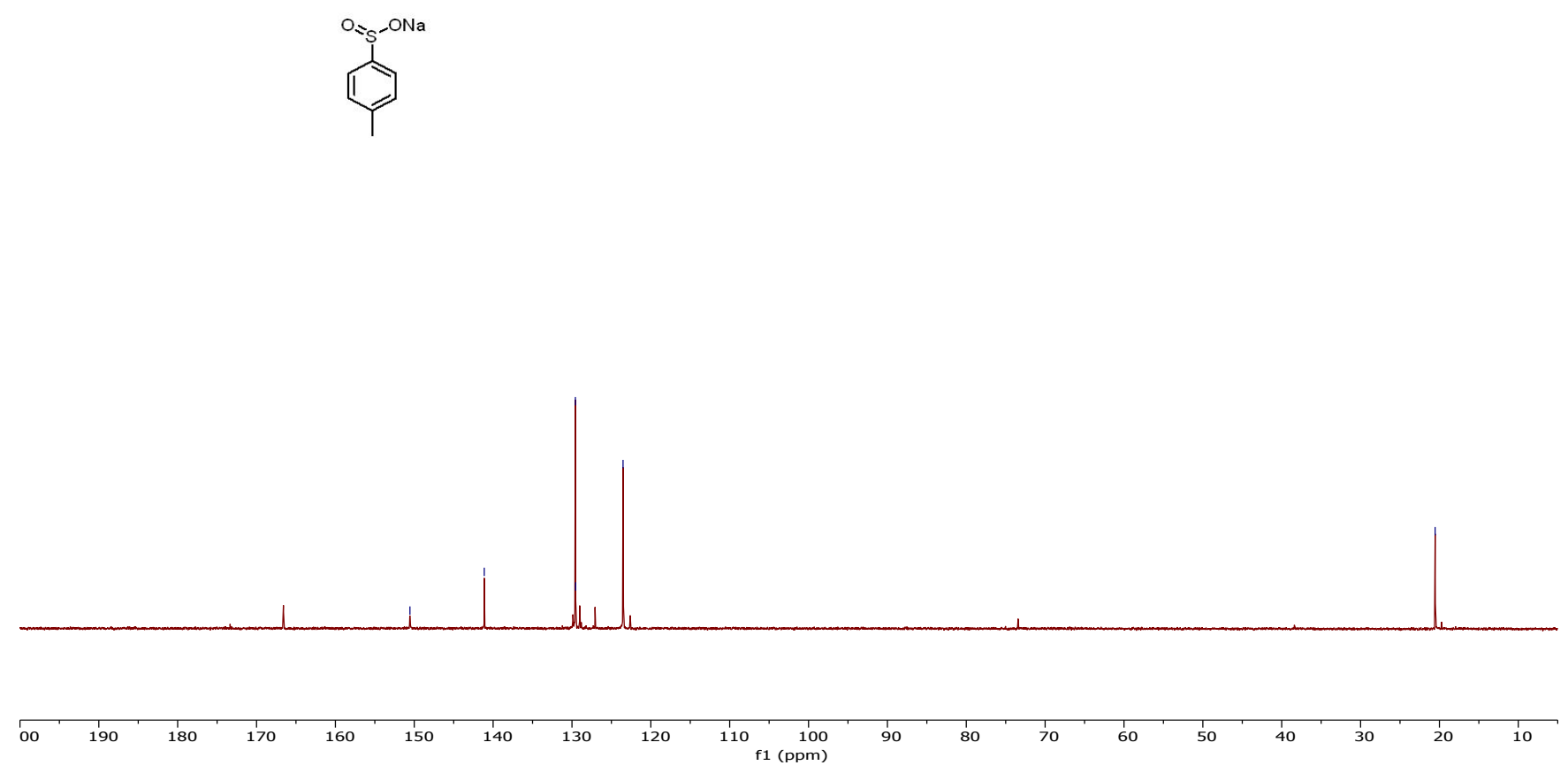

S46 


\section{Crystallogiphic Date (CCDC 2081409)}

Crystal structure and data of 3s (Displacement ellipsoids are drawn at the 50\% probability level). The crystal was grown from hexane and DCM. $19 \mathrm{mg}$ of 3s was dissolved in hexane and DCM $(1 / 5,12 \mathrm{~mL})$ and the solvent was evaporated slowly in a room atmospher. A suitable crystal was selected and mounted on a glass fibre with Fomblin oil and placed on a Agilent Gemini E diffractometer with a duel source $(\mathrm{Cu}$ at zero) equipped with an AtlasS2 CCD area detector. Crystallographic data (CCDC 2081409) can be obtained free of charge from the Cambridge Crystallographic DataCentre.

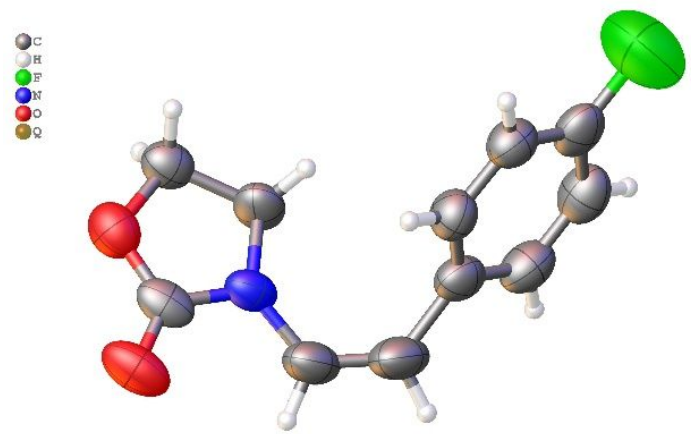

$\begin{array}{ll}\text { Empirical formula } & \mathrm{C}_{11} \mathrm{H}_{10} \mathrm{FNO}_{2} \\ \text { Formula weight } & 207.20 \\ \text { Temperature/K } & 293(2) \\ \text { Crystal system } & \text { monoclinic } \\ \text { Space group } & \mathrm{P} 2{ }_{1} / \mathrm{c} \\ \mathrm{a} / \AA & 12.9986(18) \\ \mathrm{b} / \AA & 6.5690(8) \\ \mathrm{c} / \AA & 12.8594(14) \\ \alpha /{ }^{\circ} & 90 \\ \beta /{ }^{\circ} & 96.191(12) \\ \gamma /{ }^{\circ} & 90 \\ \text { Volume } / \AA^{3} & 1091.6(2) \\ \mathrm{Z} & 4 \\ \rho_{\text {calc }} / \mathrm{cm}^{3} & 1.261 \\ \mu / \mathrm{mm}^{-1} & 0.099 \\ \mathrm{~F}(000) & 432.0 \\ \text { Crystal size } / \mathrm{mm}^{3} & 0.160 \times 0.150 \times 0.130 \\ \text { Radiation } & \mathrm{MoK} \alpha(\lambda=0.71073)\end{array}$

$2 \Theta$ range for data collection $/{ }^{\circ} 6.306$ to 51.99

Index ranges

Reflections collected

$-16 \leq \mathrm{h} \leq 15,-6 \leq \mathrm{k} \leq 8,-15 \leq 1 \leq 15$

7589

$2119\left[\mathrm{R}_{\mathrm{int}}=0.0301, \mathrm{R}_{\text {sigma }}=0.0288\right]$

Independent reflections

Data/restraints/parameters

Goodness-of-fit on $\mathrm{F}^{2}$

$2119 / 1 / 136$

1.079

Final $\mathrm{R}$ indexes $[\mathrm{I}>=2 \sigma(\mathrm{I})] \quad \mathrm{R}_{1}=0.0815, \mathrm{wR}_{2}=0.2283$

Final $\mathrm{R}$ indexes [all data] $\quad \mathrm{R}_{1}=0.1146, \mathrm{wR}_{2}=0.2559$

Largest diff. peak/hole / e $\AA^{-3} 0.21 /-0.55$ 